\title{
Translation-rotation coupling, phase transitions, and elastic phenomena in orientationally disordered crystals
}

\author{
R. M. Lynden-Bell \\ University Chemical Laboratory, Lensfield Road, Cambridge CB2 1EW, United Kingdom
}

\author{
K. H. Michel
}

Department of Physics, University of Antwerp, 2610 Wilrijk, Belgium

\begin{abstract}
Many of the properties of orientationally disordered crystals are profoundly affected by the coupling (known as translation-rotation coupling) between translation displacements and molecular orientation. The consequences of translation-rotation coupling depend on molecular and crystal symmetry, and vary throughout the Brillouin zone. One result is an indirect coupling between the orientations of different molecules, which plays an important role in the order/disorder phase transition, especially in ionic orientationally disordered crystals. Translation-rotation coupling also leads to softening of elastic constants and affects phonon spectra. This article describes the theory of the coupling from the point of view of the microscopic Hamiltonian and the resulting Landau free energy. Considerable emphasis is placed on the restrictions due to symmetry as these are universal and can be used to help one's qualitative understanding of experimental observations. The application of the theory to phase transitions is described. The softening of elastic constants is discussed and shown to be universal. However, anomalies associated with the order/disorder phase transition are shown to be restricted to cases in which the symmetry of the order parameter satisfies certain conditions. Dynamic effects on phonon spectra are described and finally the recently observed dielectric behavior of ammonium compounds is discussed. Throughout the article examples from published experiments are used to illustrate the application of the theory including well known examples such as the alkali metal cyanides and more recently discovered orientationally disordered crystals such as the fullerite, $\mathrm{C}_{60}$.
\end{abstract}

\section{CONTENTS}

List of Symbols

I. Orientationally Disordered Crystals

II. Description of Molecular Orientation

A. Rotator functions for linear molecules

B. Nonlinear molecules

C. Molecular density functions

D. Collective rotator functions

E. The use of discrete variables

III. Microscopic Description of Translation-Rotation Coupling

A. Orientation dependent potential

B. Displacement variables

C. Orientational interactions on a deformable lattice

D. The relative phases of the coupled displacement and orientational waves

E. Coupling at low $\mathbf{k}$ values

F. Coupling to acoustic phonons

G. Coupling at larger $\mathbf{k}$ values

H. Pseudospin description of translation-rotation coupling

I. Single-molecule description of translation-rotation coupling

J. Form of the potential energy

IV. Free Energies and Susceptibilities

A. The Landau free energy

B. Calculation of the Helmholtz free energy and the susceptibilities from the microscopic Hamiltonian

C. Mean-field approximation

V. Phase Transitions

A. The alkali metal cyanides and superoxides

B. Ammonium halides and sodium nitrite

C. $\mathbf{C}_{60}$ and related systems

VI. Softening of Elastic Constants

A. Symmetry of elastic constants

B. Softening of elastic constants $\quad 750$

C. Temperature dependence $\quad 752$

D. Some examples 753

E. Mixed crystals $\quad 755$

VII. Dynamic Effects and Phonons 756

VIII. Dielectric Behavior of Crystals with Deformable Ions 757

IX. Concluding Remarks $\quad 759$

References

759

\section{LIST OF SYMBOLS}

$c_{\mu \mu^{\prime}}\left(\mathbf{X}_{n n^{\prime}}\right)$

$c_{i j}$

$C_{\Gamma}$

$D(\mathbf{k})$

$\mathcal{D}_{L}^{m k}$

$F_{\text {n }}(\mathbf{Q})$

$g_{L}$

G

$j$

$J$ with elements $J_{\mu \mu^{\prime}}(\mathbf{k})$ term in the expansion of $V_{n n^{\prime}}$ in terms of rotator functions [Eq. (3.2)]

in Sec. VI-elastic constant elastic constant for strains of symmetry $\Gamma$ [Eq. (6.5)] dressed dynamical matrix [Eq. (6.6)]

Wigner rotation matrix element molecular structure factor $[\mathrm{Eq}$. (2.50)]

molecular form factor for a particular $L$ value [Eq. (2.40)] fictitious field used in deriving expressions for the free energy (Sec. III)

self-coupling matrix [Eq. (4.17)] the matrix representing the direct orientational coupling between the collective rotator 


\section{$J^{\prime}$ \\ $K_{L i}(\Omega)$ \\ $\mathbf{k}$ \\ $M$ \\ $m_{i}$
$\mathbf{n}$
$\mathbf{Q}$
$S_{\mu}=S_{L}^{\tau}$}

$\mathcal{S}(\mathbf{Q})$

$u$

$v$

$v_{a}^{R}$

$-v^{\dagger} \cdot M^{-1} \cdot v$

$V_{c}$

$V^{c}, V^{R R}, V^{T R}, V^{T R R}$

$X$

$\mathbf{X}_{n n^{\prime}}$

$Y_{L}^{m}$

$\alpha_{L}^{m \tau}$ functions $\widetilde{\Delta}_{\mu}(\mathbf{k})$

orientational coupling matrix

including direct and indirect

coupling terms [Eq. (4.4)]

cubic harmonic [examples of

symmetry-adapted functions in group $O_{h}$-Eqs. (2.4), (2.5)]

wave vector

dynamical matrix describing

phonon frequencies in the ab-

sence of translation-rotation

coupling [Eq. (3.48)]

mass of $i$ th molecule or ion

label for an atom

scattering vector

symmetry-adapted function

made from a linear combina-

tion of $L$ spherical harmonics

with symmetry species $\tau$

structure factor in $\mathrm{x}$-ray or

neutron scattering

mass weighted displacement of center of mass

translation-rotation coupling

matrix describing the coupling

between center-of-mass displace-

ments and rotator functions

[Eq. (3.21)]

coefficient in the expansion of

the orientational crystal field

potential in terms of totally

symmetric rotator functions $\Delta_{a}$

[Eq. (3.9)]

indirect coupling matrix

volume of the unit cell

terms in the total potential energy that are linear or bilinear in orientational variables $R$ and translation displacements $T$ an effective rotation-rotation coupling matrix [Eq. (7.7)] vector between centers of mass of molecules $n$ and $n^{\prime}$ spherical harmonic function coefficient in the transformation between spherical harmonics and symmetry-adapted functions These coefficients form an orthonormal set [Eq. (2.6)] rotator function [Eq. (2.21)] collective rotator function elastic strain tensor (Sec. VI) label for symmetry species in the molecular point group of rotator variables (Secs. II-V) relaxation matrix for rotator variables (Sec. VII) composite label for a rotator or

$$
\begin{aligned}
& \rho\left(r_{S}, \theta_{S}, \phi_{S}\right) \\
& \rho_{\text {mol }}\left(r_{M}, \theta_{M}, \phi_{M}\right)
\end{aligned}
$$$$
\rho_{L}^{\tau}(r)
$$$$
\tau
$$$$
\chi_{\mu \mu^{\prime}}(\mathbf{k})
$$$$
\chi_{\mu \mu^{\prime}}^{0}
$$$$
\widetilde{\Xi}_{\mu} \text { and } \Xi_{\mu}
$$$$
\Omega=(\theta, \phi)
$$

$$
\omega=(\alpha, \beta, \gamma)
$$

\section{ORIENTATIONALLY DISORDERED CRYSTALS}

A crystal that contains molecules or molecular ions is more complex than one containing only atoms or atomic ions because the individual species have orientation as well as position. This allows the existence of new types of phases, liquid crystals and orientationally disordered crystals. Orientationally disordered crystals are less well known than liquid crystals, but are fairly common in nature. Many of their properties are affected by translation-rotation coupling, which is the subject of this article.

What is an orientationally disordered crystal and when do such phases occur? In a normal crystal both the positions and orientations of the molecules from which the crystal is made are ordered. Instantaneous molecular positions are always close to those of some completely ordered ideal structure that corresponds to a local minimum of the potential energy. The deviations are the result of thermal excitation of phonon vibrational modes, the amplitudes of which are small. In a liquid there is neither translational nor orientational order. Between the crystal and liquid phases it is possible to find partially ordered phases, either liquid crystals in which most or all the translational order is destroyed but orientational or- 
der remains, or orientationally disordered crystals in which there is translational order with orientational disorder. Which (if either) of these intermediate phases occurs depends on the shapes of the molecules concerned and the relative importance of the isotropic and anisotropic parts of the intermolecular potential. Frenkel and Mulder (1985) investigated the phase diagram of hard spheroids and showed how the appearance of these phases of intermediate order depends on the axial ratio. Their phase diagram shows that orientationally disordered crystalline phases are to be expected when the molecules are nearly spherical. Often the disordered phase has a simple structure such as face-centered-cubic, although the lower symmetry of the polyatomic species is generally not compatible with the high site symmetry. This incompatibility is resolved by the presence of orientational disorder, which may be accompanied by rapid reorientations. A general account is given in a book by Parsonage and Staveley (1978). Table I lists some examples of orientationally disordered phases. These fall into two classes, molecular crystals such as methane and sulphur hexafluoride, which are sometimes known as plastic crystals (Timmermans, 1961), and ionic crystals such as the ammonium halides and alkali metal cyanides. An example of current interest is solid $\mathbf{C}_{60}$ (Krätschmer et al., 1990), with almost spherical molecules of icosahedral symmetry (Kroto et al., 1985), which has an orientationally disordered phase at room temperature.

It should be emphasized that in all examples of both orientationally disordered crystals and liquid crystals the phase is stabilized by the entropic contribution to the free energy. At low enough temperatures when the internal energy dominates the free energy, the stable phase is a completely ordered crystal, and one property of particular interest is the phase transition from the orientationally ordered to the disordered phase. As the temperature of an orientationally disordered crystal is lowered, a phase transition takes place to a more ordered phase that is characterized by a lower symmetry crystal structure and the presence of long range orientational order. Although the transition is usually of first order, the structures of the two phases can be related by a continuous path from the disordered phase to the ordered phase. Sometimes there are several successive transitions, for example sodium nitrite $\left(\mathrm{NaNO}_{2}\right)$ transforms first into an incommensurate phase and then into a ferroelectric phase (Sawada et al., 1958; Tanisaki, 1961); the alkali metal cyanides (for a review see Lüty, 1981) transform first into a (ferroelastic) phase with "quadrupolar" order in which there is still head-to-tail disorder of the $\mathrm{CN}^{-}$ions, and then at a considerably lower temperature into an antiferroelectric ordered phase.

Many properties of an orientationally disordered crystal such as elastic constants and phonon dispersion curves are affected by the nature and degree of disorder present. In some materials one finds anomalous temperature dependences, while in others the temperature dependence is normal, but the values are different from what one would expect, while in yet other materials the same property is completely normal. The elastic constants of a crystal are a measure of its stiffness under different kinds of stress (shear or elongation), and may be affected by changes in molecular orientations (Michel and Naudts, 1977a, 1977b). Figure 7 shows some of the elastic constants of sodium and potassium cyanide as a function of temperature (Haussühl et al., 1977). The values of the bulk elastic constant (or compressibility), $B=c_{11}+2 c_{12}$, for both sodium and potassium cyanide behave normally in the sense that they decrease as the temperature increases. Their values at $300 \mathrm{~K}$ are comparable with those for $\mathrm{NaCl}$ and $\mathrm{KBr}$. However, the other elastic constants are lower than expected (especially the constant $c_{44}$

TABLE I. Orientationally disordered crystals.

\begin{tabular}{|c|c|c|c|c|}
\hline Substance & Crystal structure & $\begin{array}{l}\text { Position of transition } \\
\text { in } \mathbf{k} \text { space }\end{array}$ & $\begin{array}{l}T-R \text { coupling } \\
\text { involved }\end{array}$ & Order parameter \\
\hline$M C N$ & $\mathrm{NaCl}(\mathrm{fcc})$ & $\Gamma$ & yes & $L=2 ; T_{2 g}$ \\
\hline $\mathrm{MO}_{2}$ & $\mathrm{NaCl}(\mathrm{fcc})$ & Z.B. $^{a}$ & yes & $L=2 ; E_{g}$ \\
\hline $\mathrm{NH}_{4} \mathrm{Br}, \mathrm{I}$ & $\mathrm{CsCl}$ & $M^{\mathrm{a}}$ & yes & $L=3 ; A_{2 u}$ \\
\hline $\mathrm{NH}_{4} \mathrm{Cl}$ & $\mathrm{CsCl}$ & $\Gamma$ & no & $L=3 ; A_{2 u}$ \\
\hline $\mathrm{NaNO}_{3}, \mathrm{CaCO}_{3}$ & Rhombohedral & $Z^{\mathrm{a}}$ & no & $L=3 ; A_{2}$ \\
\hline $\mathrm{NaNO}_{2}$ & tetragonal & $\xi \mathrm{a}^{*}$ & yes & $L=1 ; B_{1 u}$ \\
\hline $\mathrm{CH}_{4}, \mathrm{CBr}_{4}$ & fcc & Z.B. ${ }^{a}$ & no & $L=3 ; T_{2 u}$ \\
\hline $\mathrm{CCl}_{4}$ & fcc & Z.B. ${ }^{\mathrm{a}}$ & no & $L=3 ; T_{2 u}$ \\
\hline Adamantane & fec & Z.B. $^{a}$ & no & $L=4$ \\
\hline $\mathrm{SF}_{6}$ & fec & Z.B. ${ }^{a}$ & no & $L=4$ \\
\hline$t$ butyl bromide & fcc & Z.B. ${ }^{a}$ & no & $L=3 ; T_{2 u}$ \\
\hline $\mathrm{C}_{60}$ & fec & Z.B. ${ }^{a}$ & no & $L=10,6 ; T_{2 g}$ \\
\hline urea/paraffin & $\begin{array}{c}\text { hexagonal } \\
\text { honeycomb }\end{array}$ & Z.B. ${ }^{a}$ & yes & $L=2 ; E_{2 g}$ \\
\hline
\end{tabular}

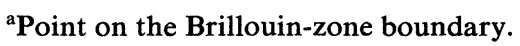


which corresponds to shear deformations of $T_{2 g}$ symmetry) and decrease as the temperature is lowered. In both materials the $T_{2 g}$ component eventually reaches a value close to zero, at which point the crystal becomes unstable and there is a weak first order phase transition to an ordered phase. This temperature is just below the temperature of the phase change to the orientationally ordered phase, and it is clear that the anomalous behavior of the elastic constants is closely associated with this phase change. It is not only the $T_{2 g}$ elastic constant that behaves anomalously; the $E_{g}$ constant also decreases with temperature in both these materials. It would seem that, if one could avoid the $T_{2 g}$ catastrophe on lowering the temperature, the crystal would become unstable to an $E_{g}$ distortion at a somewhat lower temperature and change to a different ordered phase (Michel and Naudts, 1977a, 1977b; Sahu and Mahanti, 1982). An example in which the elastic constants decrease with increasing temperature in a normal way, but have anomalous values, are the carbon tetrahalides and methane. In these materials it is the $E_{g}$ elastic constant that is lower than expected (Zuk et al., 1989, 1990; Rand and Stoicheff, 1982). We shall see that this is due to translation-rotation coupling, but that the coupling involved is unconnected with the phase transition to the ordered state.

Another striking example of the influence of translation-rotation coupling can be seen in the phonon dispersion curves. The curves in Fig. 1 are for cyanides (Rowe et al., 1975) and superoxides (Wakabayashi et al., 1982), and the unusual feature is the upwards curvature seen in some of the acoustic branches. This is the result of a k-dependent softening of the phonons due to this coupling. In these particular examples the softening is greatest near the zone center and the branches curve upwards as the softening decreases.

Most of the anomalies in crystal properties can be understood in terms of translation-rotation coupling. What is translation-rotation coupling? It is the interaction between center-of-mass displacements and large amplitude orientational motion that may occur in molecular crystals or crystals with molecular ions, which results in a change of orientational order (Rowe et al., 1975; Michel and Naudts, 1977a, 1977b; Press et al., 1979; Mahanti and Sahu, 1982; Sahu and Mahanti, 1982). It arises from terms in the Hamiltonian in which the orientation dependence of the potential of a molecule changes as a result of the relative displacement of the centers of mass of pairs of molecules. All orientationally disordered crystals show some degree of translation-rotation coupling for some phonons, but the importance of the coupling varies. We shall discuss the symmetry arguments that can be used to determine which phonons are coupled to what kind of order. This article describes the theory of this coupling and the way in which it can be used to explain elastic properties, phase transitions, phonon spectra, and dielectric measurements in orientationally disordered crystals. A particularly important aspect of the application of the theory is the use of symmetry; we demonstrate how symmetry arguments can be used to predict such things as which orientationally disordered materials may show anomalies in elastic constants and at what points in the Brillouin zone phonons will be softened and phase transitions encouraged. As always in physics and chemistry, symmetry alone cannot give the whole answer. The possibility or impossibility of an interaction is determined by symmetry, but the strength of the interaction, if allowed, depends on the intermolecular potential. We shall discuss how translation-rotation coupling parameters can be related to the intermolecular potential.

The most interesting and dramatic of translation-
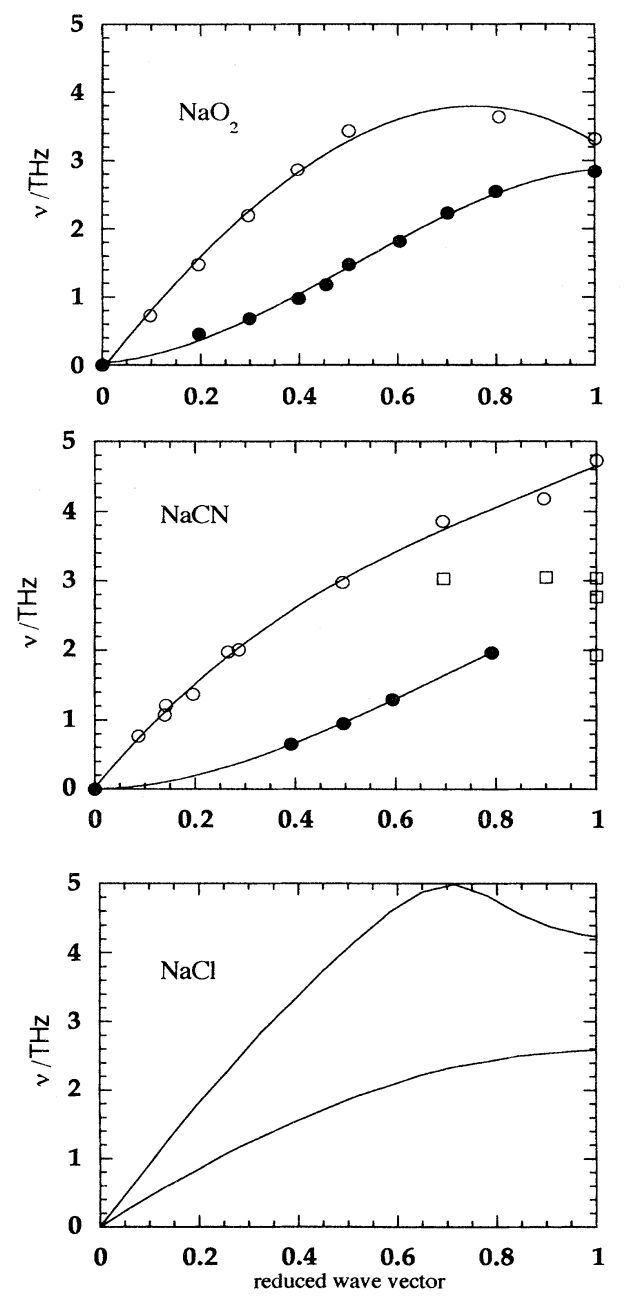

FIG. 1. Phonon-dispersion curves for sodium cyanide (data from Rowe et al., 1975), sodium superoxide (data from Wakabashi et al., 1982), and sodium chloride along the [100] direction. All these data are at room temperature. Open circles, the longitudinal branch; filled circles, the transverse branch. Notice how the transverse acoustic branches of the orientationally disordered crystals have an upward curvature at low wave vectors, a shape that is not seen in normal crystals such as sodium chloride, and that is a signature of translation-rotation coupling. 
rotation coupling-phenomena occur at phase transitions. If the orientational order parameter corresponding to the ordered phase couples with a phonon, the result is a mixed order-disorder/displacive phase transition in which the molecules or ions of the lattice simmultaneously move and disorder. The corresponding mixed phononorientation static and dynamic correlation functions are accessible to experiment and to molecular-dynamics calculations (Lynden-Bell et al., 1983, 1984). In Table I we show for which of the substances translation-rotation coupling is involved in the order-disorder transition. It is noteworthy that most of the examples come from ionic compounds rather than molecular crystals. We shall discuss the reasons for this later.

Translation-rotation coupling affects some phonons directly, as can be observed in inelastic neutron diffraction (Rowe et al., 1975; Loidl et al., 1980) or, in the long wavelength limit, in Brillouin spectra (Bischofsberger and Courtens, 1975; Krasser et al., 1976; Boissier et al., 1978). Effects due to translation-rotation coupling also appear in Raman scattering (Dultz, 1974; Fontaine and Pick, 1979; Toupry et al., 1983) and in ultrasonic experiments (Haussühl, 1973). Depending on the time scale of molecular reorientation, theory predicts either softening and damping of phonons or the appearance of a relaxation response (centered at $\omega=0$ ) together with a phonon response at the "bare" frequency (Yamada et al., 1974b; Courtens, 1976; Michel and Naudts, 1978). These effects have been confirmed experimentally (Yamada et al., 1974a; Rowe et al., 1978).

In this paper we discuss the details of the symmetry of orientational coordinates and order parameters, symmetry restrictions on translation-rotation coupling, and apply this to phonons, elastic constants, and phase transitions. Most of the examples that we use to illustrate the principles involve cubic crystals, but we make a few remarks about sodium nitrite and nitrate, which are particularly interesting systems with noncubic lattices. For the most part we restrict consideration to bilinear coupling between translational and orientational coordinates. Higher-order coupling terms have proved to be important in some materials. For example, in compressible magnets (Wagner and Swift, 1970), there is a coupling term that is quadratic in the disorder coordinate (spin) and linear in displacements. Similar higher-order coupling terms are found to be important in perovskites (Slonczewski and Thomas, 1969) and in other minerals (Salje, 1985; Carpenter, 1988). The role of the couplings between order parameter fluctuations and lattice strains in the theory of light scattering in crystals has been investigated by Ginzburg et al. (1980).

We are interested in phenomena associated with large amplitude motion and shall not treat librational motion specifically, which is known to be relevant in the ordered phases of molecular crystals. In the rest of the paper we use the term molecule to denote both an uncharged species such as $\mathrm{CBr}_{4}$ and molecular ions such as $\mathrm{NH}_{4}^{+}$, $\mathrm{CN}^{-}$, etc.

\section{DESCRIPTION OF MOLECULAR ORIENTATION}

In this section we introduce two alternative sets of variables with which to describe molecular orientation in orientationally disordered crystals. These variables will be used to describe both static and dynamic properties and are needed to develop the theory of translationrotation coupling. Both sets of variables are symmetry adapted in the sense that they are chosen to transform according to the symmetry species of the relevant symmetry group. The two different kinds are rotator functions $\Delta$, and pseudospin functions $\Xi$. The pseudospin or discrete functions arise from an extreme picture of an orientationally disordered crystal with molecules hopping between a small number of distinct but symmetrically equivalent orientations related by the symmetry operations of the crystal site group (for a review see Press, 1981). This is a good description if the crystal field is very strong. For a derivation of the crystal field we refer to Sec. III A. More usually, however, there is a wide distribution of orientations around certain preferred directions, and the probability function for molecular orientation must be described by a continuous function rather than by a series of possible discrete orientations. X-ray and neutron-diffraction studies, in particular, showed the need to take into account the continuous nature of the orientation distribution function (Atoji, 1958; KurkiSuonio, 1967; Seymour and Pryor, 1970; Press and Hüller, 1973a; Rowe et al., 1973). For example, in $\mathrm{CBr}_{4}$, although the preferred orientations are the six " $D_{2 h}$ " orientations shown in Fig. 2, there is a considerable probability of finding molecules at intermediate orientations (Dolling et al., 1979; More et al., 1980, 1984). The
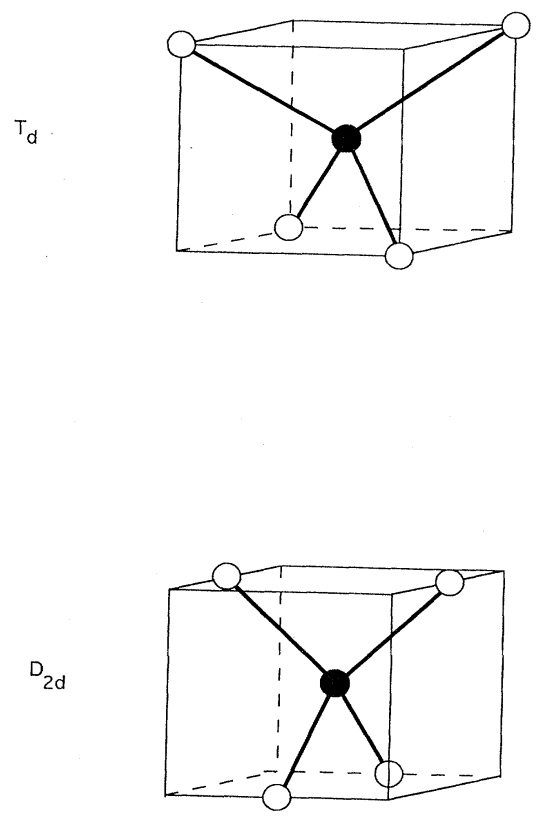

FIG. 2. The $T_{d}$ and $D_{2 d}$ orientations of tetrahedral molecules in a cubic environment. 


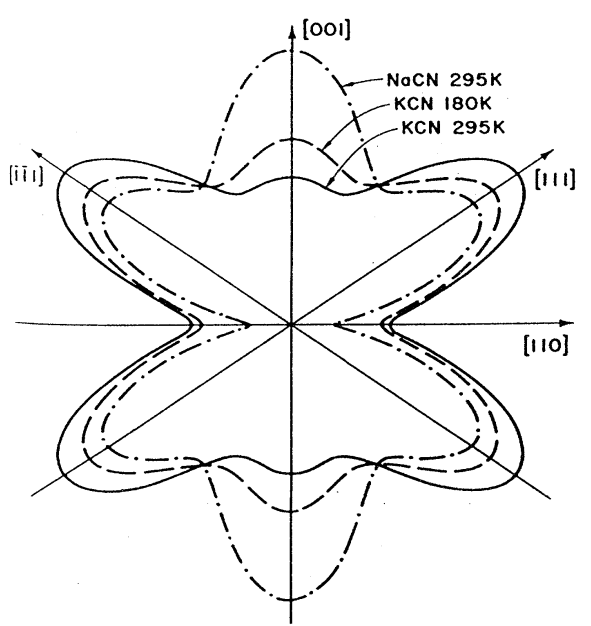

FIG. 3. Orientational distribution function of $\mathrm{CN}^{-}$in $\mathrm{NaCN}$ from Rowe et al. (1973).

orientational distribution in sodium and potassium cyanide is far from corresponding to a series of discrete sites, although it is by no means spherical. Figure 3 (taken from Rowe et al., 1973) shows orientational distributions determined by fitting neutron-scattering data to an expansion in the first three cubic harmonics.

Rotator functions can describe continuous orientational distributions and provide the basis of a more general description of the theory of phenomena observed in orientationally disordered crystals. For this description we use symmetry-adapted rotator functions, $\Delta$. These were introduced by James and Keenan (1959) in the form of tetrahedral rotator functions for the description of the orientational phase transitions in solid heavy methane.

\section{A. Rotator functions for linear molecules}

The rotator functions for linear molecules are symmetry-adapted linear combinations of spherical harmonics. The orientation of a linear molecule, considered as a rigid body, relative to crystal axes may be described by two polar angles $(\theta, \phi)=\Omega$. Any function of the orientation of a linear molecule may be described by expanding in terms of spherical harmonics $Y_{L}^{m}(\Omega)$. For example, the probability distribution function for molecular orientation can be expanded in this way:

$$
f(\theta, \phi)=1 / 4 \pi+\sum_{L, m} c_{L}^{m} Y_{L}^{m}(\theta, \phi), \quad L \neq 0,
$$

and, since the $Y_{L}^{m}$ form a complete orthonormal set, the coefficients are given by

$$
c_{L}^{m}=\int f(\Omega) Y_{L}^{m *}(\Omega) d \Omega
$$

Here, and in the following, we adopt the notation and conventions for $Y_{L}^{m}$ used in standard books on angular momentum in quantum mechanics (e.g., Edmonds, 1960;
Brink and Satchler, 1968) and in Vol. C of the International Crystallographic Tables (1992). Bradley and Cracknell (19.72) use a different convention in which the signs of spherical harmonics with positive odd values of $m$ are reversed.

The advantages of these expansions are twofold: first, if the orientation is fairly uniform (which is true if the anisotropy of the local environment is weak), a few terms suffice, and secondly, the coefficients are independent of one another. Moreover, we can simplify the expansion and reduce the number of terms necessary to describe the orientational distribution function by using the symmetry of the system. We shall define rotator functions, $S_{\mu}$, which are linear combinations of spherical harmonics that transform according to the various symmetry species of the crystal site symmetry group. The thermally averaged probability distribution function transforms according to the symmetric representation of the symmetry group of the site (Press and Hüller, 1973a; Yvinec and Pick, 1980) so that the only nonzero terms in the expansion (2.1) are the coefficients of those symmetry-adapted rotator functions which are unchanged by the symmetry operations of the site group. For octahedral symmetry these are just the "kubic harmonics" introduced by Bethe (Bethe, 1929; Von der Lage and Bethe, 1947) for the description of the crystal field due to valence electrons in solids, and used by many others since. The first applications to molecular crystals goes back to Devonshire (1936), who described the motion of a linear molecule in a field of cubic symmetry by an expansion in terms of surface harmonics of cubic symmetry.

In addition to providing a convenient and accurate description of the orientational distribution in the disordered phase, we shall show that rotator functions provide order parameters to describe the transition to a more ordered and less symmetric phase as the temperature is lowered. These order parameters are averages of rotator functions that are not totally symmetric with respect to the space group of the disordered phase, and we shall see that the change in symmetry at the phase transition determines which $S_{\mu}$ functions provide the appropriate order parameters.

Let us consider the example of linear molecules in an octahedral environment such as one finds in the disordered phases of alkali metal cyanides (Lüty, 1981) and sodium superoxides (Bösch and Känzig, 1975; Zielinski and Parlinski, 1984). The distribution function must be unchanged under symmetry operations of the molecule and under symmetry operations of the site. Considering the molecule first, if it is centrosymmetric only coefficients of even- $L$ spherical harmonics exist, otherwise all $L$ values are allowed by the molecular symmetry. However, if the site symmetry is octahedral $\left(O_{h}\right.$ - the most common symmetry in orientationally disordered crystal phases) only combinations of spherical harmonics that transform as $A_{1 g}$ in $O_{h}$ are allowed for linear molecules. Thus expression (2.1) for the orientational distribution function becomes 


$$
f=1 / 4 \pi+\sum_{L, a} c_{L}^{a} S_{L}^{a}, \quad L \neq 0,
$$

where the rotator functions $S_{L}^{a}$ for linear molecules are combinations of spherical harmonics of order $L$, which are invariant under the operations of the site group $O_{h}$. They are the cubic harmonics mentioned earlier that are the best known examples of symmetry-adapted functions. They exist for all even- $L$ values except $L=2$. For instance, for $L=4$ and $L=6$ we have the cubic harmonics $K_{L i}$ of $A_{1 g}$ symmetry, which can be written in terms of Cartesian coordinates in the crystal axes $x=\sin \theta \cos \phi$, $y=\sin \theta \sin \phi, z=\cos \theta$ of a unit vector along the molecular axis (or direction cosines) as

$$
\begin{aligned}
& K_{41}(\Omega)=\frac{5}{4}\left[\frac{21}{4 \pi}\right]^{1 / 2}\left(x^{4}+y^{4}+z^{4}-\frac{3}{5}\right), \\
& K_{61}(\Omega)=\frac{231}{8}\left[\frac{26}{4 \pi}\right]^{1 / 2}\left(x^{2} y^{2} z^{2}+\frac{1}{22}\left[K_{41}\right]-\frac{1}{105}\right),
\end{aligned}
$$

where the notation $\left[K_{41}\right]=\left(x^{4}+y^{4}+z^{4}-\frac{3}{5}\right)$ is used.

It is also possible to define symmetry-adapted functions for symmetry species other than the totally symmetric representation, and we use the notation $S_{\mu}$ and the name "symmetry adapted function" for a combination of spherical harmonics of order $L$,

$$
S_{\mu}=S_{L}^{\tau}=\sum_{m=-L}^{L} Y_{L}^{m} \alpha_{L}^{m \tau}
$$

which transforms according to the $p$ th component of the $n$th occurrence of irreducible representation $\Gamma$ of the point group $P$ under consideration. $\tau$ is a composite label for $(P, \Gamma, p, n)$ and $\mu$ for $(\tau, L)$. The cubic harmonics $K_{L i}$ are special cases of symmetry-adapted functions.

The symmetry-adapted functions $S$ form a complete set in the space spanned by $\Omega=(\theta, \phi)$ when all $L$ and $\tau$ are included. For each $L$ the transformation described by the coefficients, $\alpha_{L}$, is unitary. The inverse of Eq. (2.6) reads

$$
Y_{L}^{m}=\sum_{\tau} S_{L}^{\tau}\left(\alpha_{L}^{m \tau}\right)^{*}
$$

Values of $\alpha_{L}$ are tabulated for a selection of point groups and $L$ values by Bradley and Cracknell (1972). Unfortunately they use a different convention for the spherical harmonics, which means that the signs of $\alpha_{L}^{m \tau}$ for positive odd values of $m$ must be reversed. For $L=2$, we have $2 L+1=5$ symmetry-adapted functions, a doublet that transforms with $E_{g}=E$ symmetry, $\mu=\left(O_{h}, E_{g}, p, L=2\right), p=1,2$, and a triplet that transforms with $T_{2 g}$ symmetry so that $\mu=\left(O_{h}, T_{2 g}, p, L=2\right)$ with $p=1,2,3$. A convenient way of writing these is in terms of the Cartesian components $x, y, z$ introduced above. The two $E_{g}$ symmetry-adapted functions are

$$
S_{2}^{E_{g}, 1}(\Omega)=\left[\frac{5}{16 \pi}\right]^{1 / 2}\left(3 z^{2}-1\right)=Y_{2}^{0},
$$

$$
S_{2}^{E_{g}, 2}(\Omega)=\left[\frac{15}{16 \pi}\right]^{1 / 2}\left(x^{2}-y^{2}\right)=\left(Y_{2}^{2}+Y_{2}^{-2}\right) / \sqrt{2},
$$

and the three $T_{2 g}=f$ functions are

$$
\begin{aligned}
& S_{2}^{f, 1}(\Omega)=\left[\frac{15}{4 \pi}\right]^{1 / 2} y z=i\left(Y_{2}^{1}+Y_{2}^{-1}\right) / \sqrt{2}, \\
& S_{2}^{f, 2}(\Omega)=\left[\frac{15}{4 \pi}\right]^{1 / 2} z x=\left(-Y_{2}^{1}+Y_{2}^{-1}\right) / \sqrt{2}, \\
& S_{2}^{f, 3}(\Omega)=\left[\frac{15}{4 \pi}\right]^{1 / 2} x y=i\left(Y_{2}^{2}-Y_{2}^{-2}\right) / \sqrt{2} .
\end{aligned}
$$

In the case of an asymmetric linear molecule (a dipolar molecule!), the lowest relevant value of $L$ is 1 and we have a triplet of functions with $\mu=\left(O_{h}, T_{1 u}, p, L=1\right)$, $p=1,2,3$ :

$$
\begin{aligned}
& S_{1}^{t, 1}(\Omega)=\left[\frac{3}{4 \pi}\right]^{1 / 2} x=\left(-Y_{1}^{1}+Y_{1}^{-1}\right) / \sqrt{2}, \\
& S_{1}^{t, 2}(\Omega)=\left[\frac{3}{4 \pi}\right]^{1 / 2} y=i\left(Y_{1}^{1}+Y_{1}^{-1}\right) / \sqrt{2}, \\
& S_{1}^{t, 3}(\Omega)=\left[\frac{3}{4 \pi}\right]^{1 / 2} z=Y_{1}^{0} .
\end{aligned}
$$

All these functions are real and have been normalized so that the integral of their square over all directions is equal to unity.

\section{B. Nonlinear molecules}

The situation for nonlinear molecules is similar, although somewhat more complex, as three Euler angles $(\omega=\alpha, \beta, \gamma)$ are needed to describe the orientation of a nonlinear molecule relative to a fixed frame such as the crystal axes. One solution for the description of the orientational distribution function has been to use sums of cubic harmonics for equivalent bonds (Klein et al., 1983). A more general method is to construct rotator functions, which are functions of the Euler angles and which transform in a definite way under the symmetry operations of the site and the molecule.

First it is necessary to determine what the relevant symmetry group is. It has three kinds of operations, rotations of the molecule that leave it in equivalent positions, rotations of the site, and simultaneous improper rotations (including reflections and inversions) of both site and molecule, which leave the system in an equivalent configuration (Pick and Yvinec, 1980; Yvinec and Pick, 1980). The resulting symmetry group, which we shall term the true site-molecule group, is discussed in more de- 
tail in the above papers and by Breymann and Pick (1988). However, for most practical purposes, it is easier to consider the group formed by taking the product sitemolecule group formed from the direct product of the site and molecular point groups and to classify functions according to their behavior in these two latter groups separately. One must remember, however, that the reduction in symmetry to the true site-molecule group (which is a subgroup of the product site-molecule group) could in principle cause additional mixing or splitting of degeneracies. We know of no such examples. The probability distribution function for the orientation of the undistorted molecule, for example, transforms as the totally symmetric representation of the true site-molecule group. As long as we are considering undistorted molecules, all properties are invariant under the symmetry operations of the molecule group alone, and so the distribution function is also invariant under all the operations of the site group. As a result it transforms according to the totally symmetric representation of the product site-molecule group. An exception to this would occur for optically active molecules that have no reflections or inversion elements of symmetry. In this somewhat esoteric case the distribution function is only invariant under the rotation operations of the site group. However, as far as we are aware there are no examples known of orientationally disordered crystals with disordered optically active molecules; moreover, the site symmetry in the disordered phase is almost always centrosymmetric. For the rest of this article we shall assume that the crystal has centrosymmetric sites and the molecule has reflection or inversion symmetry operations or both, and we shall use the product site-molecule group to classify and construct rotator functions.

We now construct rotator functions from linear combinations of the Wigner rotation matrix elements $D_{L}^{m k}$ (see Edmonds, 1960, or Brink and Satchler, 1968). The functions $D_{L}^{m k}$ are the three-dimensional equivalents of the spherical harmonics, and form a complete set of functions in the space of the three Euler angles. The rotator functions formed from them are analogous to the symmetry-adapted $S$ functions already introduced for linear molecules, and we shall see the notation $\Delta_{\mu}$ for them. For an early use of rotator functions for the description of tetrahedral molecules in a cubic crystal, we refer to James and Keenan's work (1959) on $\mathrm{CD}_{4}$.

The Wigner matrices $\mathscr{D}_{L}$ form a $(2 L+1)$ dimensional representation of the rotation group generated from a basis of spherical harmonics by the rotation operators $\widehat{R}(\omega)$, which relate the molecule-fixed coordinate frame to the site-fixed frame. Suppose we start by orienting the molecule in the "standard position," in which its axis system $(\mathbf{X}, \mathbf{Y}, \mathbf{Z})$ coincides with the site axes $(\mathbf{x}, \mathbf{y}, \mathbf{z})$, and consider a function such as the spherical harmonic $Y_{L}^{k}\left(\theta_{M}, \phi_{M}\right)$ in the molecule. Now we rotate the molecule from this standard position. In the molecular frame $Y_{L}^{k}$ is unchanged, but in the laboratory frame the function is changed to

$$
\begin{aligned}
\widehat{R}(\omega) Y_{L}^{k}\left(\Omega_{M}\right) & =Y_{L}^{k}\left[\widehat{R}^{-1}(\omega) \Omega_{M}\right] \\
& =\sum_{m=-L}^{L} Y_{L}^{m}\left(\Omega_{S}\right) D_{L}^{m k}(\omega),
\end{aligned}
$$

where $\Omega_{M}$ and $\Omega_{S}$ are polar angles in the molecular and site frame, respectively. In Eq. (2.6) we defined symmetry-adapted functions $S_{L}^{\tau}$ that were linear combinations of spherical harmonics which transform as a symmetry species $\tau$ of the site group. We can now do the same for the molecule, taking linear combinations of spherical harmonics referred to the molecular axis system which transform as the symmetry species $\lambda$ of the molecular point group:

$$
S_{L}^{\lambda}\left(\Omega_{M}\right)=\sum_{k=-L}^{L} Y_{L}^{k}\left(\Omega_{M}\right) \alpha_{L}^{k \lambda}
$$

When the molecule is rotated, these are changed:

$$
\widehat{R}(\omega) S_{L}^{\lambda}\left(\Omega_{M}\right)=\sum_{k=-L}^{L} \sum_{m=-L}^{L} Y_{L}^{m}\left(\Omega_{S}\right) \mathcal{D}_{L}^{m k}(\omega) \alpha_{L}^{k \lambda} .
$$

The resulting spherical harmonics $Y_{L}^{m}\left(\Omega_{S}\right)$ in the site frame are now rewritten [using Eq. (2.7)] as linear combinations of site symmetry-adapted functions $S_{L}^{\tau}\left(\Omega_{S}\right)$, which transform according to the $\tau$ th representation of the symmetry group of the site so that

$$
\widehat{R}(\omega) S_{L}^{\lambda}\left(\Omega_{M}\right)=\sum_{\tau, m, k} S_{L}^{\tau}\left(\Omega_{S}\right)\left(\alpha_{L}^{m \tau}\right)^{*} D_{L}^{m k}(\omega) \alpha_{L}^{k \lambda} .
$$

This equation shows how the symmetry-adapted functions $S_{L}^{\lambda}\left(\Omega_{M}\right)$ in the molecule-fixed axes (classified under the molecular point group) are related to the symmetryadapted functions $S_{L}^{\tau}\left(\Omega_{S}\right)$ in the site-fixed axes (classified according to the site symmetry group). We can define the transformation by

$$
\widehat{R}(\omega) S_{L}^{\lambda}\left(\Omega_{M}\right)=\sum_{\tau} S_{L}^{\tau}\left(\Omega_{S}\right) \Delta_{L}^{\tau \lambda}(\omega) .
$$

The functions $\Delta_{L}^{\tau \lambda}(\omega)$ are the rotator functions for nonlinear molecules, which we shall use to describe orientational properties and, in particular, translation-rotation coupling. As they relate symmetry-adapted functions in the molecule and in the site, they transform according to the symmetry species $\tau$ of the site point group and $\lambda$ of the molecular point group. The value of a rotator function of a particular molecule depends on the instantaneous orientation of that molecule relative to the site. From the previous two equations we see

$$
\Delta_{\mu}=\sum_{k=-L}^{L} \sum_{m=-L}^{L} \alpha_{L}^{m \tau *} D_{L}^{m k}(\omega) \alpha_{L}^{k \lambda},
$$

where to simplify the notation we have introduced a composite label $\mu=(L, \tau, \lambda)$ for the order of spherical harmonics $L$, the symmetry species under operations of the site point group $\tau$, and the symmetry species under operations of the molecular point group $\lambda$. We note that the coefficients $\alpha$ are usually the square roots of simple 
numbers. For example from Eqs. (2.8)-(2.15) for symmetry-adapted functions in the octahedral group we find values such as $1, \pm 1 / \sqrt{2}, \pm i / \sqrt{2}$.

We shall need some properties of the rotator functions. In all the examples that we shall consider, the point groups of both the site and the molecule have symmetry species with real characters. This means that both the symmetry-adapted functions $S_{\mu}$ and the rotator functions $\Delta_{\mu}$ are real. The rotator functions form a complete set in the space spanned by the Euler angles and are normalized according to

$$
\int \Delta_{\mu^{\prime}}^{*} \Delta_{\mu} d \omega=\delta_{\mu \mu^{\prime}} \frac{8 \pi^{2}}{(2 L+1)} .
$$

The set of $(2 L+1)^{2}$ rotator functions for a given value of $L$ form a unitary matrix relating the two sets of symmetry-adapted $S_{L}$ functions. The inverse transformation (from the site frame to the molecular frame) is given by

$$
\begin{aligned}
\hat{R}^{-1}(\omega) S_{L}^{\tau}\left(\Omega_{S}\right) & =\sum_{\lambda} S_{L}^{\lambda}\left(\Omega_{M}\right)\left(\Delta_{L}^{-1}\right)^{\lambda \tau} \\
& =\sum_{\lambda} S_{L}^{\lambda}\left(\Omega_{M}\right) \Delta_{L}^{\tau \lambda *}(\omega) .
\end{aligned}
$$

Rotator functions can be used to describe any angledependent physical property of the molecule as seen from crystal axes. For example, the orientational probability distribution function may be written in terms of those $\Delta$ functions, $\Delta_{L}^{a a}$, which are totally symmetric in the site molecule group (Press and Hüller, 1973a; Yvinec and Pick, 1980):

$$
f(\omega)=1 / 8 \pi^{2}+\sum_{L, a} A_{L}^{a} \Delta_{L}^{a a}(\omega) .
$$

The sizes of the coefficients can be determined using the orthonormality condition [Eq. (2.22)]

$$
\begin{aligned}
A_{L}^{a} & =\frac{(2 L+1)}{8 \pi^{2}} \int f(\omega) \Delta_{L}^{a a *}(\omega) d \omega \\
& =\frac{(2 L+1)}{8 \pi^{2}}\left\langle\Delta_{L}^{a a *}\right\rangle,
\end{aligned}
$$

showing that the sizes of the coefficients $A_{L}^{a}$ describe the nonuniformity of the distribution. This is entirely analogous to the treatment of the orientational distribution function of a linear molecule in terms of the symmetryadapted functions $S_{L}^{a}$ [Eqs. (2.1)-(2.3)], the difference being that now we need three angles to describe the orientation of a nonlinear molecule.

To illustrate the properties of rotator functions and the way in which they are constructed we consider the hypothetical example of a $\mathrm{NO}_{2}^{-}$ion in an octahedral environment. The shape of the nitrite ion (Fig. 4) is an isosceles triangle with the $\mathbf{N}$ atom at the apex. It has $C_{2 v}$ symmetry. We start by considering spherical harmonics in the molecule-fixed axis system $(X, Y, Z)$ and construct linear combinations of these functions that transform as

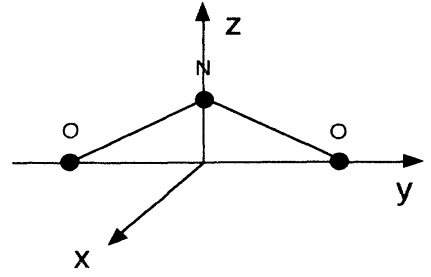

FIG. 4. The geometry of the nitrite ion.

the various irreducible representations of the molecular point group $\left(C_{2 v}\right)$. For $L=1$ and $L=2$ we have for $A_{1}$ symmetry

$$
\begin{aligned}
& S_{1}^{A_{1}}=Y_{1}^{0}, \\
& S_{2}^{A_{1}, 1}=Y_{2}^{0}, \\
& S_{2}^{A_{1}, 2}=\left(Y_{2}^{2}+Y_{2}^{-2}\right) / \sqrt{2},
\end{aligned}
$$

where there are two different combinations of $L=2$ functions that transform according to the totally symmetric representation, which we have distinguished by the superscripts 1 and 2 .

The symmetry-adapted functions for the other three symmetry species in the molecular point group are

$$
\begin{aligned}
& S_{1}^{B_{1}}=\left(-Y_{1}^{1}+Y_{1}^{-1}\right) \sqrt{2}, \\
& S_{2}^{B_{1}}=\left(-Y_{2}^{1}+Y_{2}^{-1}\right) / \sqrt{2}, \\
& S_{1}^{B_{2}}=i\left(Y_{1}^{1}+Y_{1}^{-1}\right) / \sqrt{2}, \\
& S_{2}^{B_{2}}=i\left(Y_{2}^{1}+Y_{2}^{-1}\right) / \sqrt{2}, \\
& S_{2}^{A_{2}}=i\left(-Y_{2}^{2}+Y_{2}^{-2}\right) / \sqrt{2} .
\end{aligned}
$$

All these combinations are real, in agreement with the properties already noted.

The relevant symmetry-adapted rotator functions for the site symmetry group $\left(O_{h}\right)$ have already been given in Eqs. (2.8)-(2.15). The $\Delta$ functions relate the two sets of symmetry-adapted functions, the molecular set $S_{L}^{\lambda}$ in the molecular frame and $S_{L}^{\tau}$ in the site frame. They are constructed from the Wigner rotation matrices according to Eq. (2.21). As an example we calculate the rotator function $\Delta_{\mu}$, which relates the $L=2$ functions that have $B_{1}$ symmetry in the molecular frame to those that transform in the site symmetry group as the second row of $E_{g}$ [i.e., as $S_{2}^{E_{g}, 2}$, see Eq. (2.9)]. This has $\mu=\left[O_{h}, E_{g}(2), C_{2 v}, B_{1}, L=2\right]$ and is

$$
\begin{aligned}
\Delta_{\mu} & =\int S_{2}^{E_{g}, 2} \hat{R}(\omega) S_{2}^{B_{1}} d \Omega \\
& =\frac{1}{2} \int\left(Y_{2}^{2}+Y_{2}^{-2}\right) \hat{R}(\omega)\left(-Y_{2}^{1}+Y_{2}^{-1}\right) d \Omega,
\end{aligned}
$$

which can be rewritten in terms of Wigner rotation matrix elements as

$$
\Delta_{\mu}=\frac{1}{2}\left\{-\mathscr{D}_{2}^{21}-\mathscr{D}_{2}^{-21}+\mathscr{D}_{2}^{2-1}+\mathscr{D}_{2}^{-2-1}\right\} .
$$


Expressions for the Wigner rotation functions in terms of Euler angles are given in textbooks on angular momentum theory (for example, Brink and Satchler, 1968), but note that those given by Bradley and Cracknell (1972) may have different signs and for consistency must be used with their definition of spherical harmonics. Substituting we obtain

$$
\Delta_{\mu}=\frac{1}{2} \sin 2 \beta \cos 2 \alpha \cos \gamma-\sin \beta \sin 2 \alpha \sin \gamma .
$$

Quaternion parameters are often used in computer simulations of molecules in liquids or crystals (Hüller and Kane, 1974; Allen and Tildesley, 1987) and provide a convenient route to constructing rotator functions. Expressions relating these to Wigner rotation matrix elements are given by Lynden-Bell and Stone (1989).

In many ways the most instructive way of writing these functions is in terms of direction cosines of the angles between the molecule and site axes. Using the relationships between direction cosines and Wigner rotation matrix elements given by Lynden-Bell and Stone (1989), one finds for this example

$$
\Delta_{\mu}=X_{x} Z_{x}-X_{y} Z_{y}
$$

where $X_{z}$ is the direction cosine between the moleculefixed $X$ axis and the site-fixed $z$ axis. One can see immediately that this function transforms in the same way as the product $X Z$ under the molecular symmetry operations and the same way as $x^{2}-y^{2}$ under the site symmetry operations. Inspection of character tables shows that, as expected, $X Z$ transforms as $B_{1}$ in the molecular point group $C_{2 v}$, and $x^{2}-y^{2}$ transforms as $E_{g}$ under the symmetry operations of the site group $O_{h}$. The construction of rotator functions in terms of direction cosines of molecule-fixed axes relative to the laboratory axis system has been used to calculate orientational order and translation-rotation coupling in molecular-dynamics calculations, which used the constraint method rather than quaternions (Ferrario et al., 1987; Lynden-Bell et al., 1989).

In most of the applications discussed in this article we are concerned only with undistorted molecules and so with functions that are unchanged under the symmetry operations of the molecule and that transform according to the totally symmetric representation of the molecular group. In our example of a nitrite ion, such functions must transform according to $A_{1}$ in the molecular point group $C_{2 v}$. There is one such function for $L=1$, namely, $Y_{1}^{0}$. This generates three rotator functions for $L=1$ that are identical with those for a linear molecule [Eqs. (2.13)-(2.15)], if one identifies $x, y$, and $z$ as the direction cosines $Z_{x}, Z_{y}$, and $Z_{z}$ between the direction of the molecular dipole and the site axes. For $L=2$, each of the two $A_{1}$ symmetry-adapted combinations of spherical harmonics generates a set of rotator functions. The set based on $S_{2}^{A_{1}, 1}=Y_{2}^{0}$ is identical to those for a linear molecule if the dipole direction is substituted for the direction of the molecular axis. For example, for
$\mu=\left[O_{h}, E_{g}(2), C_{2 v}, A_{1}, 1, L=2\right]$, where 1 labels the first occurrence of symmetry $A_{1}$,

$$
\Delta_{\mu}=Z_{x} Z_{x}-Z_{y} Z_{y}
$$

This set of functions gives information about the orientation and properties of the molecular dipole. The second set, labeled by 2 , is based on $S_{2}^{A_{1}, 2}=\left(Y_{2}^{2}+Y_{2}^{-2}\right) / \sqrt{2}$ and gives information about the orientation of the triangle of atoms about the dipole direction. This gives rise to a new set of rotator functions that show the effects of the molecular shape. For example, for $\mu=\left[O_{h}, E_{g}(2), C_{2 v}, A_{1}, 2, L=2\right]$ we have

$$
\Delta_{\mu}=\left(X_{x} X_{x}-X_{y} X_{y}-Y_{x} Y_{x}+Y_{y} Y_{y}\right) / 2,
$$

which transforms as $x^{2}-y^{2}$ under the operations of the site group and as $X^{2}-Y^{2}$ under the operations of the molecular group.

The $S$ functions previously defined in Eq. (2.6) for linear molecules are special cases of these $\Delta$ functions for molecular point groups $C_{\infty v}$ and $D_{\infty h}$, and in the rest of this article we shall not normally distinguish between linear and nonlinear molecules. It should be noted that, as a matter of convenience, the $\Delta$ functions for linear molecules have been integrated over the redundant angle $\gamma$, which gives the factor of $2 \pi$ difference between the constant terms in Eq. (2.3) for linear molecules and in (2.23) for nonlinear molecules.

One reason that these rotator functions are particularly useful is that many molecular properties of interest transform as a particular $L$ value. For example, dipole moments transform as $L=1$ so that all properties of molecular dipole moments, irrespective of molecular symmetry, are described by the subset of rotator functions with $L=1$. Similarly, molecular quadrupole moments and the anisotropic part of molecular polarizabilities are described by rotator functions with $L=2$.

\section{Molecular density functions}

Much information about molecular orientation in plastic crystals is obtained from neutron and $\mathrm{x}$-ray scattering. These techniques do not recognize the presence of molecules, but only the average distribution of scattering material in the crystal. Consequently the rotator functions are not determined directly but have to be reconstructed. The data from neutrons (or $\mathrm{x}$ rays) can be interpreted in terms of the average density of scattering material around all equivalent sites in the crystal. The contribution from a molecule at a particular site depends on the distribution of scattering material of the molecule in its molecule-fixed axes (which we assume is constant) and the instantaneous orientation of that particular molecule. Thus the average scattering density $\rho\left(r, \Omega_{S}\right)$ at a set of equivalent sites is

$$
\rho\left(r, \Omega_{S}\right)=\int P(\omega) \hat{R}(\omega) \rho_{\mathrm{mol}}\left(r, \Omega_{M}\right) d \omega,
$$

where $P(\omega)$ is the temperature-dependent probability of 
finding a molecule at orientation $\omega$, and $\rho_{\text {mol }}\left(r, \Omega_{M}\right)$ is the distribution of scattering material of a molecule relative to its axis system. For simplicity, we have assumed here that the origins of both axis systems coincide with the center of mass of the molecule. Both $\rho\left(r, \Omega_{S}\right)$ and $\rho_{\text {mol }}\left(r, \Omega_{M}\right)$ can be expanded in symmetry-adapted functions. The site density is totally symmetric under the symmetry operations of the site, and the molecular density is totally symmetric under the symmetry operations of the molecule. Hence (Seymour and Pryor, 1970; Rowe et al., 1973; Press and Hüller, 1973a)

$$
\begin{aligned}
& \rho\left(r, \Omega_{S}\right)=\sum_{L, \tau=A_{1}} \rho_{L}^{\tau}(r) S_{L}^{\tau}\left(\Omega_{S}\right) \\
& \rho_{\mathrm{mol}}\left(r, \Omega_{M}\right)=\sum_{L, \lambda=A_{1}} \rho_{\mathrm{mol}, L}^{\lambda}(r) S_{L}^{\lambda}\left(\Omega_{M}\right) .
\end{aligned}
$$

Equation (2.20) shows that the symmetry-adapted functions $S_{L}^{\lambda}$ in the molecular frame are related to those in the site frame $\left(S_{L}^{\tau}\right)$ by the rotator functions so

$$
\rho_{L}^{\tau}(r)=\left\langle\Delta_{L}^{\tau \lambda}\right\rangle \rho_{\mathrm{mol}, L}^{\lambda}(r),
$$

where the angular brackets denote the thermal average. Thus if we know the scattering density of a particular kind of molecule at a given site, the thermal averages of the rotator functions can be found.

We shall now describe the way in which the molecular scattering density can be constructed and introduce the useful concept of the molecular form factors $g_{L}$. Atoms of a given kind, which are in equivalent positions, lie at a constant distance from the center of mass of the molecule. In order to keep the notation simple, we restrict ourselves to one type of atom, for example, the $\mathrm{Br}$ atoms of $\mathrm{CBr}_{4}$ or the carbon nuclei of $\mathrm{C}_{60}$. The position of the $v$ th atom with respect to the center of mass is described by the radius vector $\mathbf{d}_{v}$, whose polar angles are $\boldsymbol{\Omega}_{v}=\left(\theta_{v}, \phi_{v}\right)$ in a molecule-fixed frame. The first task is to find the density distribution in the molecule-fixed axis frame. The orientational distribution of the nuclei can be written as the sum of delta functions

$$
\sum_{v} \delta\left(\Omega-\Omega_{v}\right)=\sum_{L, k} Y_{L}^{k *}(\Omega)\left[\sum_{v} Y_{L}^{k}\left(\Omega_{v}\right)\right]
$$

It is convenient to define

$$
c_{L}^{k}=\sum_{v} Y_{L}^{k}\left(\Omega_{v}\right)
$$

As a consequence of molecular symmetry, the only values of $L$ that can occur in this expansion are those that contain a totally symmetric symmetry species of the molecular point group. Only even $L$ values are allowed if the molecule has a center of symmetry. We have already seen this for symmetric linear molecules. If the molecule is tetrahedral, only the values $L=0,3,4$. . are allowed by symmetry (Press, 1973b), while for $\mathbf{S F}_{6}$, an octahedral molecule, one has $L=0,4,6 \ldots$, and the centrosym- metric icosahedral molecule $\mathrm{C}_{60}$ has $L=0,6,10 \ldots$ (Michel et al., 1992).

We now write the orientational distribution of the nuclei in terms of the symmetry-adapted functions

$$
\sum_{v} \delta\left(\Omega-\Omega_{v}\right)=\sum_{L} g_{L} S_{L}^{A}(\Omega)
$$

where we have introduced molecular form factors $g_{L}$ (Michel and Parlinski, 1985) as coefficients in this expansion. These are useful in many applications. Comparing the last two equations we see that

$$
g_{L}=\sum_{k} \alpha_{L}^{A k *} c_{L}^{k}=\sum_{v} S_{L}^{A}\left(\Omega_{v}\right),
$$

where $\alpha_{L}^{k A}$ are the coefficients relating symmetry-adapted functions to spherical harmonics defined in Eq. (2.6). It can be shown that, for the totally symmetric symmetry species,

$$
\alpha_{L}^{k A}=c_{L}^{k} /\left[\sum_{k} c_{L}^{k *} c_{L}^{k}\right]^{1 / 2}
$$

giving an alternative expression for $g_{L}$,

$$
g_{L}=\left(\sum_{k} c_{L}^{k *} c_{L}^{k}\right)^{1 / 2}
$$

The contribution of these nuclei to the scattering density $\rho_{\text {mol }}[$ Eq. (2.35)] is

$$
\rho_{L, \mathrm{~mol}}^{A}(r)=g_{L} b \delta(r-d),
$$

where $b$ is the scattering length of the type of nucleus concerned.

If there is more than one type of atom in the molecule, a form factor $g_{L}(v)$ must be computed for each type of atom $v$. There is no unique way of defining either the site axis system or the molecular axis system. If the standard orientation is not obvious (e.g., for tetrahedral molecules), it should be specified, although the values of $g_{L}$ are independent of the choice.

We are now in the position to write down the reorientational contribution to the average scattering density. Equations (2.36) and (2.43) give

$$
\rho_{L}^{\tau}(r)=\sum_{v}\left\langle\Delta_{L}^{\tau A}\right\rangle g_{L}(v) b_{v} \delta\left(r-d_{v}\right) .
$$

Thermal fluctuations in the center of mass positions smooth the delta functions into gaussian distributions whose widths are given by the Debye-Waller factors (Press et al., 1979).

\section{Collective rotator functions}

So far we have restricted ourselves to the description of single-particle properties. In order to describe properties such as phase transitions, lattice vibrations, or elastic constants, it is necessary to define collective orientational 
functions that contain the translational symmetry of the crystal as well as the site molecule symmetry. We introduce Fourier transforms of the $\Delta$ functions

$$
\widetilde{\Delta}_{\mu}(\mathbf{k})=N^{-1 / 2} \sum_{n} \Delta_{\mu}\left(\omega_{n}\right) \exp \left(-i \mathbf{k} \cdot \mathbf{X}_{n}\right)
$$

where the sum is over $\Delta$ functions of molecules $n$ whose ideal lattice positions are $\mathbf{X}_{n} ; N$ is the total number of molecules. For convenience of notation, we restrict ourselves here to crystals that contain only one molecular unit per primitive unit cell in the orientationally disordered phase, but the construction can readily be generalized. We use a tilde to denote functions such as these that are defined in reciprocal space ( $\mathbf{k}$ space) and the same symbol without a tilde to refer to the corresponding function in real space, and we note that the complex conjugate of a function in $\mathbf{k}$ space is the same as the function evaluated at $-\mathbf{k}$, for example, $\widetilde{\Delta}_{\mu}^{*}(\mathbf{k})=\widetilde{\Delta}_{\mu}(-\mathbf{k})$. It is convenient to introduce a vector notation for the multicomponent functions $\Delta_{\mu}$. We define a column vector $\widetilde{\Delta}(\mathbf{k})$ with components $\widetilde{\Delta}_{\mu}(\mathbf{k})$. By taking the Hermitian conjugate, we define a row vector, $\widetilde{\Delta}^{\dagger}(\mathbf{k})$, with components $\widetilde{\Delta}_{\mu}^{*}(\mathbf{k})$. As the rotator functions are real, we have

$$
\widetilde{\Delta}_{\mu}^{*}(\mathbf{k})=\widetilde{\Delta}_{\mu}(-\mathbf{k}) .
$$

Because the crystal is translationally invariant, the functions that are totally symmetric in the complete crystal space group are collective $\widetilde{\Delta}$ functions for which $\mathbf{k}=0$, and which transform as the totally symmetric representation of the site molecule group. These are the only rotator functions with nonzero averages. However, although the average value of other $\widetilde{\Delta}$ functions must be zero, fluctuations in them may (and do) occur.

One may define an orientational susceptibility matrix

$$
\chi_{\mu \mu}(\mathbf{k})=\left\langle\widetilde{\Delta}_{\mu}^{*}(\mathbf{k}) \widetilde{\Delta}_{\mu}(\mathbf{k})\right\rangle / k_{B} T,
$$

where $k_{B}$ is Boltzmann's constant and $\langle\cdots\rangle$ denotes a thermal average taken with the full Hamiltonian of the system. This is the classical statistical-mechanics expression for the susceptibility. Since we are not treating molecular crystals like $\mathbf{H}_{2}$ (Silvera, 1980; van Kranendonk, 1983), $\mathrm{CH}_{4}$ (Kataoka et al., 1973; Kobashi et al., 1984), or any other molecular crystals such as $\mathrm{O}_{2}$ and $\mathrm{N}_{2}$ (Briels et al., 1986), where quantum effects are important, but heavier molecules at reasonably high temperatures, we are in the classical rotator regime $\hbar^{2} /\left(I k_{B} T\right)<1$, where $I$ is the moment of inertia of the molecule. There are also mixed susceptibilities $\chi_{\mu \mu^{\prime}}(\mathbf{k})$,

$$
\chi_{\mu \mu^{\prime}}(\mathbf{k})=\left\langle\widetilde{\Delta}_{\mu}^{*}(\mathbf{k}) \widetilde{\Delta}_{\mu^{\prime}}(\mathbf{k})\right\rangle / k T .
$$

Mixed susceptibilities are zero unless $\mu$ and $\mu^{\prime}$ have the same symmetry in the space group. This means that they must have the same value of $\mathbf{k}$, and depending on the direction of $\mathbf{k}$ there may be further restrictions. However, the restrictions that the single-particle functions from which they are composed transform in the same way un- der the site molecule group is lifted if $\mathbf{k} \neq 0$. We shall return to this point later. Diagonal susceptibilities are always nonzero, although if we restrict our attention to rigid molecules, we shall only be concerned with fluctuations in which $\Delta_{\mu}$ transforms as the totally symmetric representation of the molecular point group. $\Delta$ functions corresponding to other molecular point group symmetries correspond to fluctuations in which the molecules are allowed to distort or vibrate. The orientational order that occurs when the temperature is lowered through a phase transition is described by an order parameter that is the average of one of the $\widetilde{\Delta}$ functions that is not totally symmetric with a particular value of $\mathbf{k}$.

A basic quantity that enters the differential cross section for $x$-ray and neutron scattering is the structure factor $\mathcal{S}(\mathbf{Q})$. This can be related to the rotator functions,

$$
\mathcal{S}(\mathbf{Q})=\sum_{\mathbf{n}} \sum_{v} b_{v} \operatorname{expi} \mathbf{Q} \cdot\left[\mathbf{X}_{\mathbf{n}}+\mathbf{d}_{\mathbf{n}}(v)\right] .
$$

Here $\hbar \mathbf{Q}$ is the momentum transferred in the scattering process, $d_{n}(v)$ specifies the position of the $v$ th atom of the molecule at the lattice site $\mathrm{n}$, and $b_{v}$ is the scattering length of atoms of type $v$. We consider the thermal average of the molecular structure factor $F_{n}(Q)$ at this site, and assume (for simplicity of notation) that all atoms are of the same type, so that

$$
\left\langle F_{\mathbf{n}}(\mathbf{Q})\right\rangle=\sum_{v} b\left\langle\operatorname{expi} \mathbf{Q} \cdot \mathbf{d}_{\mathbf{n}}(v)\right\rangle .
$$

Following the procedure of Pick and Yvinec (1980), we expand the molecular structure factor in terms of rotator functions to obtain

$$
\left\langle F_{\mathrm{n}}(\mathbf{Q})\right\rangle=4 \pi \sum_{L} b \sum_{\tau} i^{L} j_{L}(Q d)\left\langle S_{L}^{\tau}\left(\Omega_{Q}\right)\right\rangle \Delta_{L}^{\tau A}(\mathbf{n})
$$

where $j_{L}$ is a spherical Bessel function, $S_{L}^{\tau}$ is a site symmetry adapted function, and $\Omega_{Q}$ stands for the polar angles of $\mathbf{Q}$. For a set of equivalent lattice sites, the average molecular structure factor $\left\langle F_{n}(Q)\right\rangle$ is the same at all sites $\mathbf{n}$ and the structure factor is

$$
\mathcal{S}(\mathbf{Q})=\frac{(2 \pi)^{3}}{V_{c}} \sum_{\mathbf{G}} \delta(\mathbf{Q}-\mathbf{G})\langle F(\mathbf{Q})\rangle,
$$

where $\mathbf{G}$ is a reciprocal lattice vector and $V_{c}$ is the volume of the unit cell. By a suitable choice of the scattering geometry it is often possible to select a specific representation $\tau$ and hence to measure the Bragg reflections that correspond to a condensation of a particular order parameter $\left\langle\Delta_{L}^{\tau A}\right\rangle$. As an example we refer to $\mathrm{CD}_{4}$ (Press, 1973; Press and Hüller, 1974).

In later parts of the paper we shall need the values of $\chi_{\mu \mu^{\prime}}^{0}$, the single particle susceptibilities in the absence of any coupling (direct or indirect) of molecular orientation in neighboring sites 


$$
\chi_{\mu \mu^{\prime}}^{0}=\left\langle\Delta_{\mu}\left(\omega_{n}\right)^{*} \Delta_{\mu^{\prime}}\left(\omega_{n}\right)\right\rangle_{0} / k_{B} T,
$$

where the thermal average is taken with the crystal field potential at site $n$. The thermal average is zero unless $\mu$ and $\mu^{\prime}$ transform as the same representation of the site molecule group. In addition the periodicity of the crystal implies that the expression is the same for all sites in the crystal. We can therefore write

$$
\chi_{\mu \mu^{\prime}}^{0}=y_{\mu}^{0} \delta_{\mu \mu^{\prime}} / T,
$$

where the single-particle averages $y_{\mu}^{0}$ are usually only weakly temperature dependent. It is often a fair approximation to take $y_{\mu}^{0}$ to be a constant whose value depends on a particular rotator function $\mu$, so that each $\chi_{\mu \mu}^{0}$ is inversely proportional to the temperature.

\section{E. The use of discrete variables}

Translation-rotation coupling may also be formulated using collective discrete (pseudospin) variables. If the disordered molecule occupies a discrete number of orientations then one may associate a variable $\xi_{p}$ with each orientation. The value of each $\xi_{p}$ is either one or zero according to whether the orientation is occupied or not. The sum of all the discrete variables at a given site is equal to one. This is analogous to a Potts model in which each molecule has a number of possible states corresponding to the allowed orientations. Symmetry-adapted combinations of the discrete variables are then constructed using standard group theoretical projection techniques to obtain

$$
\Xi_{\mu}=\sum_{p} \alpha_{\mu p} \xi_{p} .
$$

These are the analogs of the rotator functions $\Delta_{\mu}$ introduced earlier [Eq. (2.21)]. In this case the subscript $\mu$ labels the symmetry species of the site group.

The best way to illustrate the method for using symmetry-adapted discrete variables is to consider specific examples. At the same time we shall compare the use of rotator functions for the same systems. The main examples, which we shall use here and in subsequent sections, are alkali metal cyanides, carbon tetrahalides, and ammonium halides.

First we consider $\mathrm{NH}_{4} \mathrm{Br}$. The disordered $\beta$ phase crystallizes in the $\mathrm{CsCl}$ structure with eight nearest bromide ions and six ammonium molecular ions in the next-nearest shell. There are only two distinct preferred orientations of the ammonium ion, known as the " $T_{d}$ " orientations, in which the protons point towards the corners of the cube formed by the eight nearest-neighbor bromide ions (Fig. 2). Hence the discrete description of the orientations uses just two variables (or states in the Potts model analogy) $\xi_{1}$ and $\xi_{2}$. Either $\xi_{1}=1$ and $\xi_{2}=0$ or $\xi_{1}=0$ and $\xi_{2}=1$, according to which way the molecule is oriented. In the site group $O_{h}$ we have symmetry combinations of these states

$$
\begin{aligned}
& \Xi_{A_{1 g}}=\left(\xi_{1}+\xi_{2}\right), \\
& \Xi_{A_{2 u}}=\left(\xi_{1}-\xi_{2}\right) .
\end{aligned}
$$

These functions are similar to the $\Delta_{\mu}$ rotator functions, but instead of an infinite set, there are two such functions as there are only two possible orientations. The value of $\Xi_{A_{1 g}}$ is always equal to 1 while the discrete variable $\Xi_{A_{2 u}}$ is equal to \pm 1 depending on which way the molecule is oriented. $\Xi_{A_{2 u}}$ behaves like a spin variable, and is sometimes known as a pseudospin. Collective variables can now be formed in an analogous way to that used to form $\widetilde{\Delta}(\mathbf{k})$ functions from the single-molecule functions $\Delta\left(\omega_{n}\right)$, by summing over the function for each molecule $n$ with an appropriate phase, for example,

$$
\widetilde{\Xi}_{A_{2 u}}(\mathbf{k})=N^{-1 / 2} \sum_{n} \Xi_{A_{2 u}}(n) \exp \left(-i \mathbf{k} \cdot \mathbf{X}_{n}\right) \text {. }
$$

At equilibrium in the disordered phase all sites are occupied equally, and only $\widetilde{\Xi}_{A_{1 g}}(\mathbf{k}=0)$ has a nonzero average. The order parameter for an ordered phase in which all the molecules are aligned in the same direction is the average of the $\widetilde{\Xi}_{A_{2 u}}(\mathbf{k}=0)$ coordinate. In fact the ordered phase that is reached from the disordered phase by cooling, the $\gamma$ phase, is characterized by columns of aligned ammonium molecular ions. Within each column the molecules have the same orientation, and the columns form a square array with adjacent columns containing molecules opposite orientations. The transition to this phase from the disordered phase is described by a collective pseudospin order parameter $\widetilde{\Xi}_{A_{2 u}}\left(\mathbf{k}_{M}\right)$ (Yamada et al., 1972) where $\mathbf{k}_{M}=\left(\pi / a_{0}, \pi / a_{0}, 0\right)$ is the wave vector at the $M$ point of the Brillouin-zone boundary, and $a_{0}$ is the cubic lattice constant. In the lowesttemperature $\delta$ phase all the molecules are oriented in the same way, which can be described by a collective discrete order parameter with $A_{2 u}$ symmetry at k=0. Alternatively order parameters for the two phases can be defined in terms of collective rotator functions of $A_{2 u}$ symmetry with $\mathbf{k}=\mathbf{k}_{M}$ and $\mathbf{k}=0$, respectively. The principal order parameter for either structure would have $L=3$, the lowest $L$ value allowed by the tetrahedral symmetry of the molecule.

Other discrete coordinates can be used to describe fluctuations in order and translation-rotation coupling, but can never account for a continuous distribution of orientations. In the rotator function description this is possible, and, in principle, all the $\Delta$ functions that are symmetric in the site molecule group should be included. As the $\mathrm{NH}_{4}^{+}$ion is tetrahedral, the lowest allowed rotator functions that are symmetric under the operations of the molecular group are the seven $L=3$ functions, which were originally introduced by James and Keenan (1959) for the theoretical study of phase transitions in solid $\mathrm{CD}_{4}$. In $O_{h}$ these transform as $A_{2 u}, T_{1 u}$, and $T_{2 u}$; the next-lowest rotator functions are formed from the $L=4$ harmonics and transform as $A_{1 g}, E_{g}, T_{1 g}$, and $T_{2 g}$. 
Thus the probability distribution function [Eq. (2.24)] contains $A_{1 g}$ rotator functions with $L=4,6,8, \ldots$, where the series may be terminated at a point depending on the required accuracy. The phase transition to the $\beta$ phase is described by an order parameter formed from the $L=3$ collective rotator functions with symmetry $A_{2 u}$ at the zone boundary point $\mathbf{k}_{M}, \widetilde{\Delta}_{\mu}\left(\mathbf{k}_{M}\right)$. There are suitable functions with $L=3,7$, and higher odd $L$ values. In general the most relevant order parameter is the one with the lowest symmetry-allowed value of $L$, and the higher ones may be treated as subsidiary order parameters or ignored. As already noted, there are exceptions to this, for example, the cyanides and $\mathrm{C}_{60}$, where the electronic structure of the molecule makes them more symmetric than they seem. However, interactions with the ammonium ion feel the tetrahedral symmetry, and the appropriate principal order parameters are the averages of $\widetilde{\Delta}_{\mu}\left(\mathbf{k}_{M}\right)$ with $\mu=\left(L=3, A_{2 u}\right)$. Notice that the symmetry of the order parameter is the same whether one uses the symmetry-adapted rotator function approach or the pseudospin function description, although the microscopic picture is somewhat different.

As a second example we consider $\mathrm{CBr}_{4}$ (Dolling et al., 1979; More et al., 1980; More and Fouret, 1980). The disordered phase is face-centered cubic, each molecule being in a site with $O_{h}$ symmetry with twelve nearest neighbors. Both the molecular symmetry $\left(T_{d}\right)$ and the site symmetry $\left(O_{h}\right)$ of the molecule are the same as those of the ammonium ion in our previous example, so that the rotator functions available to describe the molecular orientations are the same. The first nonzero term in the expansion of the orientational distribution function is a $\widetilde{\Delta}(\mathbf{k}=0)$ function with $L=4$, and there are possible $L=3$ order parameters based on the $A_{2 u}, T_{1 u}$, and $T_{2 u}$ symmetry combinations. At the zone center the symmetry of these order parameters in the full space group $\left(O_{h}\right)$ is the same as the site symmetry. The ordered monoclinic phase has a larger unit cell corresponding to a condensation away from the zone center.

The pseudospin model for $\mathrm{CBr}_{4}$ (Coulon and Descamps, 1980) has to be very different from that for the ammonium salt, as the $\mathrm{CBr}_{4}$ molecule has six, rather than two, favored orientations. It is constructed from six states $\xi_{j}$, corresponding to the six " $D_{2 d}$ " orientations illustrated in Fig. 2. These six discrete states transform as $A_{1 g}, E_{g}$, and $T_{2 u}$,

$$
\begin{aligned}
& \Xi_{A_{1 g}}=\sum_{j=1}^{6} \xi_{j} / \sqrt{6}, \\
& \Xi_{E_{g a}}=\left[2\left(\xi_{1}+\xi_{4}\right)-\left(\xi_{2}+\xi_{5}\right)-\left(\xi_{3}+\xi_{6}\right)\right] / \sqrt{12}, \\
& \Xi_{E_{g a}}=\left[\left(\xi_{2}+\xi_{5}\right)-\left(\xi_{3}+\xi_{6}\right)\right] / 2, \\
& \Xi_{T_{2 u a}}=\left(\xi_{1}-\xi_{4}\right) / \sqrt{2}, \\
& \Xi_{T_{2 u b}}=\left(\xi_{2}-\xi_{5}\right) / \sqrt{2}, \\
& \Xi_{T_{2 u c}}=\left(\xi_{3}-\xi_{6}\right) / \sqrt{2} .
\end{aligned}
$$

To obtain a pure orientational state, such as $\xi_{1}$, one must superimpose $E_{g}$ and $T_{2 u}$ states, showing that both order parameters are involved in the ordering process. Comparing these results with the rotator function description, we see that both $L=3\left(T_{2 u}\right)$ and $L=4\left(E_{g}\right)$ order parameters must be involved if complete order of individual molecules is to be obtained.

Although $\mathrm{CBr}_{4}$ and $\mathrm{NH}_{4} \mathrm{Br}$ have identical site symmetries and identical molecular symmetry, the nature of the ordered phase is different. This illustrates one of the limitations of group theory, namely, that it cannot predict which of many possible symmetries are important. The answer to this question depends on the intermolecular forces that determine the details of the microscopic Hamiltonian. The use of discrete variables is justified only if the crystal field, which tends to localize the molecules, is very strong.

\section{MICROSCOPIC DESCRIPTION OF TRANSLATION-ROTATION COUPLING}

In this section we study the coupling of molecular orientations with lattice displacements at the molecular level. As we have seen, translation-rotation coupling is caused by the changes in orientational potential felt by a molecule when it or nearby molecules or atoms move. The amount of this change depends on the actual strength and form of the intermolecular potentials. We shall establish a form for the microscopic Hamiltonian, which will later be used to find the free energy of the crystal and to discuss the effects of translation-rotation coupling on phase transitions and elastic constants. In this Hamiltonian the orientations are described by the rotator functions already introduced, and center-of-mass displacements by variables that we shall shortly define. There are bilinear terms in the Hamiltonian coupling pairs of those variables that transform in the same way in the crystal space group. We shall show how the coefficients in this Hamiltonian can be determined from intermolecular potentials.

This section is somewhat more mathematical, and should give the reader sufficient knowledge to carry out calculations for specific systems. On the other hand, a reader who wishes to understand the implications of translation-rotation coupling may prefer to proceed directly to Sec. IV at first reading.

The potential energy of the crystal depends on the positions and orientations of every molecule and atomic ion and is invariant under the symmetry operations of the crystal. The terms that we are interested in are fairly short range in real space and describe the interaction between a molecule and its close neighbors. The orientational distribution function depends on the changes in the average potential energy of a molecule in different orientations, while translation-rotation coupling depends on the changes in potential energy when both orientation and displacement are varied, so that we need a microscopic description of the intermolecular potential as a 
function of orientation and the way in which it varies with center-of-mass displacements.

\section{A. Orientation dependent potential}

The interaction of a pair of molecules $n, n^{\prime}$ whose centers of mass are separated by $\mathbf{X}_{n n^{\prime}}$, depends on $\mathbf{X}$ and on the orientations of the molecules. It is useful to use the crystal axes as a frame of reference for the two molecules. Because the rotator functions form a complete set in the orientational space of each molecule, this interaction can be expanded in rotator functions as (Michel and Parlinski, 1985)

$$
V_{n n^{\prime}}=\sum_{\mu \mu^{\prime}} c_{\mu \mu^{\prime}}\left(\mathbf{X}_{n n^{\prime}}\right) g_{L} g_{L^{\prime}} \Delta_{\mu}(n) \Delta_{\mu^{\prime}}\left(n^{\prime}\right),
$$

where $g_{L}$ and $g_{L}$ are the molecular form factors introduced before, and the label $\mu=L, \tau, A_{1}$, where $\tau$ is a symmetry species of the site group and $A_{1}$ is the totally symmetric symmetry species of the molecular point group. The interactions of molecules and atomic ions (if present) are also described by an equation of this form, but if species $n^{\prime}$ is spherically symmetrical then the only contribution is from $L^{\prime}=0$ with $\Delta=1$ and $g_{0}=N_{a}(4 \pi)^{-1 / 2}$, where $N_{a}=\Sigma_{v} 1$ is the total number of atoms or interaction centers in each molecule.

We shall now calculate the expansion coefficients $c_{\mu \mu^{\prime}}\left(\mathbf{X}_{n n^{\prime}}\right)$, assuming that the intermolecular potential $V_{n n^{\prime}}$ is the sum of two-body atom-atom terms $V_{\text {pair }}$ between atoms in each molecule. The form of this potential varies for interactions between different types of atom or ion. For simplicity we first calculate the interaction between two molecules each containing atoms of one type in symmetrically equivalent positions. The molecule is not necessarily linear. The interatomic potential between atoms $v$ and $v^{\prime}$ on different molecules $n$ and $n^{\prime}, V_{\text {pair }}\left(r_{v v^{\prime}}\right)$ depends on the type of atoms $v$ and $v^{\prime}$ and on the distance $\left(r_{v v^{\prime}}\right)$ between them. This distance can be reexpressed as a function of the molecular center of mass separation vector $\mathbf{X}_{n n^{\prime}}$ and the orientations of vectors $\left(\mathbf{r}_{n v}\right)$ and $\left(\mathbf{r}_{n^{\prime} v^{\prime}}\right)$ connecting each atom with the center of mass of its molecule (see Fig. 5). The orientationdependent terms can be expressed in terms of sitesymmetry-adapted functions $S_{\mu}$ [Eq. (2.6)] in which the label $\mu=(L, \tau)$ describes the $L$ value and the symmetry species in the site group and one can rewrite the interaction between the pair of atoms $v$ and $v^{\prime}$ as

$$
V_{\text {pair }}\left(r_{v v^{\prime}}\right)=\sum_{\mu \mu^{\prime}} c_{\mu \mu^{\prime}}\left(\mathbf{X}_{n n^{\prime}}\right) S_{\mu}(v) S_{\mu^{\prime}}\left(v^{\prime}\right),
$$

where we have written $S_{\mu}(v)$ for $S_{\mu}\left(\Omega_{v}\right)$. The coefficient $c_{\mu \mu^{\prime}}$ is obtained by integrating the pairwise interaction over all orientations of both radius vectors $\left(\mathbf{r}_{n v}\right)$ and $\left(\mathbf{r}_{n^{\prime} v^{\prime}}\right)$ :

$$
c_{\mu \mu^{\prime}}\left(\mathbf{X}_{n n^{\prime}}\right)=\iint V_{\text {pair }}\left(r_{v v^{\prime}}\right) S_{\mu}\left(\Omega_{v}\right) S_{\mu^{\prime}}\left(\Omega_{v^{\prime}}\right) d \Omega_{v} d \Omega_{v^{\prime}} .
$$

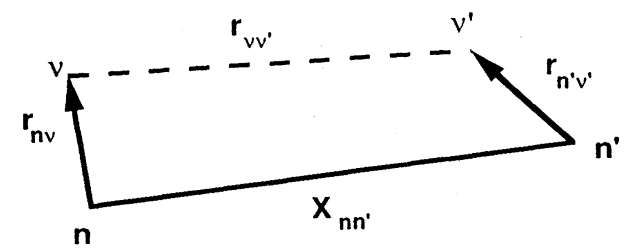

FIG. 5. The vectors used in the calculation of molecular interactions.

To obtain the interaction between the pair of molecules $n$ and $n^{\prime}$ we now sum over the interactions between all atoms in each molecule,

$$
V_{n n^{\prime}}=\sum_{\mu \mu^{\prime}} c_{\mu \mu^{\prime}}\left(\sum_{v} S_{\mu}(v)\right]\left[\sum_{v^{\prime}} S_{\mu}\left(v^{\prime}\right)\right] .
$$

The sum of $S_{\mu}$ over atoms in each molecule transforms as the totally symmetric symmetry species of the molecular point group. If the molecule is in its standard orientation, in which the molecular axis system coincides with the site axis system, this sum is equal to the form factor $g_{L}$, defined in Eq. (2.40). If the molecule is at an arbitrary orientation $\omega$, then

$$
\sum_{v} S_{\mu}(v)=g_{L} \Delta_{\mu}\left(\omega_{n}\right)
$$

where $\Delta_{\mu}$ is a rotator function [Eq. (2.21)] with label $\mu=\left(L, \tau, A_{1}\right)$ defining an $L$ value, a symmetry species $\tau$ in the site group, and symmetry species $A_{1}$ in the molecular group. Hence the instantaneous interaction between molecules with arbitrary orientation is

$$
V_{n n^{\prime}}=\sum_{\mu \mu^{\prime}} c_{\mu \mu^{\prime}}\left(\mathbf{X}_{n n^{\prime}}\right) \Delta_{\mu}(n) \Delta_{\mu^{\prime}}\left(n^{\prime}\right) g_{L} g_{L^{\prime}},
$$

where $\Delta_{\mu^{\prime}}\left(n^{\prime}\right)=\Delta_{\mu^{\prime}}\left(\omega_{n}^{\prime}\right)$. In the case of linear molecules this expression is still valid with $\Delta$ replaced by $S$. The interaction between a molecule and a spherical species is treated in exactly the same way, but the only coefficients are $c_{\mu 0}$. If there are several different kinds of atoms or interaction sites in each molecule one just adds the contributions from the different pair potentials when calculating $V_{n n^{\prime}}$.

The total potential energy of the crystal is the sum of such terms for all pairs of molecules and atomic ions in the lattice. The different terms in the expansion have different interpretations. For example, the crystal field potential $V^{R}$, which is the field on a molecule $n$ when all other molecules are at their lattice sites and spherically averaged, is obtained from the $L^{\prime}=0$ terms in the expansion, while the higher $L^{\prime}$ terms give rise to direct rotation-rotation coupling terms in the Hamiltonian, $V^{R R}$. The integrals in Eq. (3.3) are conveniently evaluated numerically. However, it is also possible to give analytical expressions for the coefficients $c_{\mu \mu^{\prime}}$ for some particular intermolecular potentials (Briels et al., 1986).

As a particular case we calculate the crystal field po- 
tential from Eq. (3.6). Molecules $n^{\prime}$ are treated in spherical approximation, so that $S_{L^{\prime}}^{\tau^{\prime}}$ is replaced by $S_{0}^{A}{ }_{1 g}=(4 \pi)^{-1 / 2}$. Since the field at site $n^{\prime}$ is totally symmetric under the site group operations, we need the expansion coefficients

$$
c_{a 0}\left(\mathbf{X}_{n n^{\prime}}\right)=\frac{1}{\sqrt{4 \pi}} \iint V_{\mathrm{pair}}\left(r_{v v^{\prime}}\right) S_{L}^{A g}(v) d \Omega_{v} d \Omega_{v^{\prime}}
$$

where $a$ stands for $\left(L, A_{1 g}, A_{1}\right)$. Observing that $\Delta_{L=0}=1$, we obtain for the total crystal field potential at site $n$

$$
V^{R}=\sum_{a} v_{a}^{R} \Delta_{a}(n)
$$

where

$$
v_{a}^{R}=\sum_{n^{\prime}} c_{a 0}\left(\mathbf{X}_{n n^{\prime}}\right) g_{L} g_{0} .
$$

The sum is taken over all species in the crystal, that is, both coions and counterions in ionic crystals. Due to translational invariance the crystal field is the same at each site. In the case of a linear molecule the $\Delta_{a}$ are cubic harmonics, while for nonlinear molecules they are cubic rotator functions (Press and Hüller, 1973a and 1973b). Crystal field potentials have been calculated for several systems, including the alkali metal cyanides (Michel and Rowe, 1985b) and neopentane (Breyman and Pick, 1989).

It is convenient to write the orientation-dependent intermolecular contributions to the potential energy of the crystal in Fourier space (reciprocal space). Using the definition of $g_{L}$ in Eq. (2.40) and making use of the translational invariance of the lattice, we obtain from Eq. (3.6)

$$
V^{R R}=\frac{1}{2} \sum_{\mathbf{k}} \tilde{\Delta}^{\dagger} \cdot J \cdot \widetilde{\Delta}
$$

where the coupling matrix for the direct rotationrotation coupling has elements

$$
J_{\mu \mu^{\prime}}(\mathbf{k})=\sum_{n^{\prime}} g_{L} g_{L^{\prime}} c_{\mu \mu^{\prime}}\left(\mathbf{X}_{n n^{\prime}}\right) \exp \left[i \mathbf{k} \cdot \mathbf{X}_{n n^{\prime}}\right]
$$

Similarly the crystal field potential may be written

$$
V^{R}=\sqrt{N} g_{0} v_{a}^{R} \widetilde{\Delta}_{a}(\mathbf{k}=0) .
$$

\section{B. Displacement variables}

The dependence of the potential energy on the centerof-mass coordinates is conveniently described using collective mass-weighted phonon displacement coordinates $\widetilde{u}(\mathbf{k})$. In order to set these up we consider the displacement $u_{i}(n)$ of molecule $n$ from its equilibrium position, $\mathbf{X}_{n}$ in direction $i$. Suppose that there are $s$ different mole- cules or ions in the unit cell, which we distinguish by labels $\kappa$. We label the molecule or molecular ion that is disordered by $\kappa=1$ and take its equilibrium position to be the origin of the unit cell (i.e., $\mathbf{r}_{1}=0$ ). We define $N_{u}=3 \mathrm{~s}$ mass-weighted collective variables by

$$
\widetilde{u}_{\rho}(\mathbf{k})=\sum_{n}\left(m_{\kappa} / N\right)^{1 / 2} u_{i}(n) \exp \left(i \mathbf{k} \cdot \mathbf{X}_{\mathbf{n}}\right),
$$

where $\rho=(\kappa, i)$ is a composite label denoting the type of molecule by $\kappa$ (e.g., ammonium or bromide in ammonium bromide) and the direction of displacement $(x, y$, or $z)$ by $i$. The sum over molecules $n$ is over all molecules of the type being considered $(\kappa)$ in the lattice, and $m_{\kappa}$ is the mass of this type of molecule.

It is then again convenient to define column and row vectors $\tilde{u}(\mathbf{k})$ and $\tilde{u}^{\dagger}(\mathbf{k})$ each with $3 s$ components $\tilde{u}_{\rho}(\mathbf{k})$ and $\tilde{u}_{\rho}^{*}(\mathbf{k})\left[=\tilde{u}_{\rho}(-\mathbf{k})\right]$, respectively. It is sometimes useful to take combinations corresponding to acoustic or optic modes, which can also be subsumed into the label $\rho$. Following Born and Huang (1956), we define three vectors, $e_{i}, i=x, y, z$ in the $3 s$-dimensional space of massweighted lattice displacements

$e_{i}=\left[\delta_{i x}\left(m_{1} / m\right)^{1 / 2}, \delta_{i y}\left(m_{1} / m\right)^{1 / 2}, \ldots, \delta_{i z}\left(m_{s} / m\right)^{1 / 2}\right]$,

where $m=\Sigma_{\kappa} m_{\kappa}$ is the mass per unit cell and $\delta$ is the Kronecker symbol. The acoustic coordinates are then given by

$$
\tilde{u}_{i}^{a c}(\mathbf{k})=\sum_{\rho} e_{i \rho} \tilde{u}_{\rho}(\mathbf{k})=\sum_{\kappa}\left(\frac{m_{\kappa}}{m}\right)^{1 / 2} \tilde{u}_{\rho}(\mathbf{k}) .
$$

If there is more than one atom of a given type in the primitive unit cell, the definitions of collective coordinates must be generalized and it is advantageous to choose combinations that transform under a given representation of the space group concerned. However, in many orientationally disordered crystals there is only one molecule of each type in the primitive unit cell, so we will not consider this complication.

\section{Orientational interactions on a deformable lattice}

In order to determine the translation-rotation coupling we need to extend the results of Sec. III.A by considering a nonrigid lattice. We start again from the expression (3.6) for the intermolecular potential of a pair of molecules. The instantaneous molecular center-of-mass positions are now given by $\mathbf{r}_{n}=\mathbf{X}_{n}+\mathbf{u}_{n}$. Expansion in terms of the lattice displacements yields to first order

$$
V=V_{0}+V^{(1)} \text {, }
$$

where $V_{0}$ is the rigid lattice contribution that has been studied in Sec. III.A, which includes the crystal field term and the direct rotation-rotation coupling term. The first-order contribution from a pair of molecules or from a molecule and a spherical ion is the derivative of Eq. 
(3.6) with respect to changes in the intermolecular displacement

$$
\begin{gathered}
V^{(1)}=\sum_{i} \sum_{n n^{\prime}} \sum_{\mu \mu^{\prime}} c_{i \mu \mu^{\prime}}^{\prime}\left(\mathbf{X}_{n n^{\prime}}\right) \Delta_{\mu}(n) \Delta_{\mu^{\prime}}\left(n^{\prime}\right) g_{L} g_{L^{\prime}} \\
\times\left[u_{i}(n)-u_{i}\left(n^{\prime}\right)\right],
\end{gathered}
$$

where $u_{i}(n)$ is the displacement of molecule (ion) $n$ in direction $i$ from its ideal position, the label $\mu=\left(L, \tau, A_{1}\right)$, and $c_{i \mu \mu^{\prime}}^{\prime}\left(\mathbf{X}_{n n^{\prime}}\right)$ is the derivative of $c_{\mu \mu^{\prime}}\left(\mathbf{X}_{n n^{\prime}}\right)$ with respect to a change of intermolecular separation in the direction $i, X_{i n n^{\prime}}$. This is calculated from Eq. (3.3). For practical calculations it is best first to take the derivative of the pair potential and then to do the integrals over orientations,

$$
\begin{aligned}
c_{i \mu \mu^{\prime}}^{\prime}\left(\mathbf{X}_{n n^{\prime}}\right)=\iint & \frac{d V_{\text {pair }}\left(r_{v v^{\prime}}\right)}{d r_{v v^{\prime}}} \frac{\partial\left(r_{v v^{\prime}}\right)}{\partial X_{i n n^{\prime}}} \\
& \times S_{\mu}\left(\Omega_{v}\right) S_{\mu^{\prime}}\left(\Omega_{v^{\prime}}\right) d \Omega_{v} d \Omega_{v^{\prime}} .
\end{aligned}
$$

Contributions to the first-order energy from different values of $L$ have different consequences. The term with $L, L^{\prime}=0$ is zero as the lattice is stable. The terms arising from $L, L^{\prime} \neq 0$ have no contributions from interactions between molecules and atomic ions. They give a trilinear interaction $V^{R R T}$, which generalizes the corresponding result for the well-known compressible Ising model (Wagner and Swift, 1970) and may cause anomalies near structural phase transitions (Ginzburg et al., 1980). Here we are mainly concerned with the translationrotation coupling term $V^{T R}$, which is obtained by taking all molecules except one (labeled $n$ ) in the spherical approximation, that is $L^{\prime}=0, L \neq 0$. This term contains contributions from both molecule-molecule interactions and from interactions of molecules with spherical ions. It can be written

$$
V^{T R}=\sum_{n, n^{\prime}} \sum_{\mu, i} c_{i \mu}^{\prime}\left(\mathbf{X}_{n n^{\prime}}\right) \Delta_{\mu}(n) g_{L} g_{0}\left[u_{i}(n)-u_{i}\left(n^{\prime}\right)\right]
$$

where we have written $c_{i \mu}^{\prime}$ for $c_{i \mu 0}^{\prime}$ to simplify the notation. In this case the expression (3.18) becomes

$$
\begin{array}{r}
c_{i \mu}^{\prime}\left(\mathrm{X}_{n n^{\prime}}\right)=\frac{1}{\sqrt{4 \pi}} \iint \frac{d V_{\mathrm{pair}}\left(r_{v v^{\prime}}\right)}{d r_{v v^{\prime}}} \frac{\partial\left(r_{v v^{\prime}}\right)}{\partial X_{i n n^{\prime}}} \\
\times S_{\mu}\left(\Omega_{v}\right) d \Omega_{v} d \Omega_{v^{\prime}} .
\end{array}
$$

The relative strengths of the translation-rotation interaction $c_{i \mu}^{\prime}\left(\mathbf{X}_{n n^{\prime}}\right)$ and the direct rotation-rotation interaction $c_{\mu \mu^{\prime}}\left(\mathbf{X}_{n n^{\prime}}\right)$ depend on the interatomic distance. Since in ionic molecular crystals such as the alkali metal cyanides or ammonium halides the distance between unlike ions is less than the distance between like ions, the bilinear translation-rotation coupling $V^{T R}$, which includes interactions between atomic ions and molecules, becomes dominant in comparison to direct cyanide-cyanide or ammonium-ammonium orientational coupling. On the other hand, in nonionic crystals the direct interaction is often more important, and the coupling $V^{R R T}$ may also play an important role. For instance, in solid $\mathrm{C}_{60}$ this coupling (Lamoen and Michel, 1993) causes a discontinuity of the cubic lattice constant (David et al., 1992; Heiney et al., 1992) at the orientational phase transition at $T_{c}=260 \mathrm{~K}$. In the rest of this article we concentrate on the bilinear translation-rotation coupling.

It is convenient to write the coupling $V^{T R}$ in Fourier space. Using the collective rotator functions defined in Eq. (2.45) and the displacement variables defined in Eq. (3.13) we obtain

$$
V^{T R}=\sum_{\mathbf{k}} \widetilde{u}(\mathbf{k})^{\dagger} \cdot v(\mathbf{k}) \cdot \widetilde{\Delta}(\mathbf{k}),
$$

where the translation-rotation coupling is described by an $N_{u} \times N_{\Delta}$ matrix $v$ with k-dependent elements that describe the strength of the coupling. We shall also define an $N_{\Delta} \times N_{u}$ matrix $v^{\dagger}$ that is related to $v$ by transposition and complex conjugation. The matrix $v$ is obtained by Fourier transforming Eq. (3.19) and adding the contributions from all pairs of molecules. We define Fourier transforms of the coefficients $c^{\prime}$ [Eq. (3.20)], describing the interaction of the molecule $n$ with species of type $\kappa$ by

$$
\widetilde{c}_{\rho \mu}^{\prime}(\mathbf{k})=\sum_{n^{\prime} \in \kappa} c_{i \mu}^{\prime}\left(\mathbf{X}_{n n^{\prime}}\right) \exp \left[i \mathbf{k} \cdot \mathbf{X}_{n n^{\prime}}\right]
$$

where the label $\rho=(i, \kappa)$ describes the direction of the displacement and the type of species concerned. In a molecular crystal with only one type of molecule with mass $m_{1}$, the matrix elements of $v$ are

$$
v_{\rho \mu}(\mathbf{k})=g_{L} g_{0}\left[\widetilde{c}_{\rho \mu}^{\prime}(\mathbf{k}=0)-\widetilde{c}_{\rho \mu}^{\prime}(\mathbf{k})\right] / m_{1}^{1 / 2}
$$

In an ionic crystal with molecules (label 1) and counterions (label 2) the coupling with the molecules is

$v_{\rho \mu}(\mathbf{k})=g_{L} g_{0}\left[\sum_{\rho^{\prime}} \widetilde{c}_{\rho^{\prime} \mu}^{\prime}(\mathbf{k}=0)-\widetilde{c}_{\rho \mu}^{\prime}(\mathbf{k})\right] / m_{1}^{1 / 2}$,

where the sum over $\rho^{\prime}$ is over displacements in the $i$ direction of species of all types (molecule and counterion). With the counterions there is no $\mathbf{k}=0$ contribution, and

$$
v_{\rho \mu}(\mathbf{k})=-g_{L} g_{0} \widetilde{c}_{\rho \mu}^{\prime}(\mathbf{k}) / m_{2}^{1 / 2} .
$$

In these expressions the label $\rho$ identifies the type of molecule ( 1 or 2 ) and the direction $i=x, y$, or $z$ of the displacement.

For linear molecules the situation is somewhat simpler. Michel and Rowe (1985a) published calculations of the translation-rotation interaction in alkali metal cyanides, thereby generalizing and extending earlier work by Michel and Naudts (1977a, 1977b), Mahanti and Sahu (1982), and Sahu and Mahanti (1982). Each cyanide ion is surrounded by a shell of six neighboring alkali ions arranged octahedrally at distance $a$, a second shell of twelve cyanide ions at $\sqrt{2} a$, a third shell of alkali ions, 
and so on. $v_{\rho \mu}$ is the sum of terms from each of these shells. The rotator functions with $L=2$ comprise a doublet of $E_{g}$ symmetry and a triplet of $T_{2 g}$ symmetry defined in Eqs. (2.8)-(2.12). The contribution from each shell to $v$ is the product (or sums of products) of an integral over orientations of molecule $n$ of the type shown in Eq. (3.20) and a matrix that contains k-dependent elements. The value of the integral is independent of the value of $\mathbf{k}$, but depends on the symmetry of the rotator function involved.

In the case of $E_{g}$ symmetry, the coupling matrix $v$ is a $6 \times 2$ matrix coupling the functions given in Eqs. (2.8) and (2.9) to $x, y$, and $z$ displacements of the alkali-metal ion (mass $m_{M}$ ) and the cyanide ion, respectively. The contribution to this matrix from the shell of nearest neighbors is

$$
v(\mathbf{k})=\frac{2 i A}{m_{M}^{1 / 2}}\left[\begin{array}{cc}
\sin k_{x} a & \sqrt{3} \sin k_{x} a \\
\sin k_{y} a & -\sqrt{3} \sin k_{y} a \\
-2 \sin k_{z} a & 0 \\
0 & 0 \\
0 & 0 \\
0 & 0
\end{array}\right]
$$

The $T_{2 g}$ coupling matrix is a $6 \times 3$ matrix and the contribution from the first shell has the form

$$
v(\mathbf{k})=\frac{2 i B}{m_{M}^{1 / 2}}\left[\begin{array}{ccc}
0 & \sin k_{z} a & \sin k_{y} a \\
\sin k_{z} a & 0 & \sin k_{x} a \\
\sin k_{y} a & \sin k_{x} a & 0 \\
0 & 0 & 0 \\
0 & 0 & 0 \\
0 & 0 & 0
\end{array}\right] .
$$

The constants $A$ and $B$ are integrals of the type shown in Eq. (3.20). The only contribution to $A$ comes from changes in which an alkali atom is displaced towards the cyanide, while $B$ arises from displacements of alkali atoms perpendicular to the direction of the central cyanide. This can be deduced by considering the vibrational normal modes of an octahedral complex (Herzberg, 1945) where the $E_{g}$ vibrations involve radial displacements while the $T_{2 g}$ vibrations have tangential displacements. The contributions from shells of ions that are further away can be found in a similar way. The integrals over molecular orientations are smaller and the matrix elements vary more rapidly with $\mathbf{k}$.

\section{The relative phases of the coupled displacement and orientational waves}

The relative phase of the interacting collective orientational and displacement variables is determined by symmetry unless the crystal or the molecule is optically active. We assume that the molecule is not optically active and the site is at a center of symmetry. As the singleparticle rotator functions $\Delta_{\mu}$ of the chosen molecule at site $n$ transform in the same way as a linear combination of spherical harmonics of order $L$, they are changed by a factor $(-1)^{L}$ when the coordinates are inverted in the site center of symmetry. For every molecule or atom $n^{\prime}$ at position $\mathbf{r}=\mathbf{X}_{\mathbf{n}}-\mathbf{X}_{\mathbf{n}^{\prime}}$ relative to the chosen one, there is another molecule of the same type at the inverse position $-r$. Since the displacements $u_{n^{\prime}}$ change sign under inversion, molecule $n^{\prime}$ and its inverse must move in opposite directions to preserve the center of symmetry and in the same direction to destroy it. Thus the coupling coefficients are obtained from Eq. (3.22),

$$
\begin{aligned}
& \widetilde{c}_{i \mu}^{\prime}(\mathbf{k})=i \sum_{n^{\prime}} c_{i \mu}^{\prime} \sin \left[\mathbf{k} \cdot \mathbf{X}_{n n^{\prime}}\right], \quad \text { even } L, \\
& \widetilde{c}_{i \mu}^{\prime}(\mathbf{k})=\sum_{n^{\prime}} c_{i \mu}^{\prime} \cos \left[\mathbf{k} \cdot \mathbf{X}_{n n^{\prime}}\right], \quad \text { odd } L .
\end{aligned}
$$

The phase difference between these expressions describes the fact that orientational variables of even $L$ couple out-of-phase with displacements, while those of odd $L$ couple with in-phase displacements. The coupling constant matrices for alkali cyanides given in Eqs. (3.26) and (3.27) provide an example of this property for even $L$.

The distinction between in-phase and out-of-phase coupling is illustrated in Fig. 6, which shows a transverse phonon in a one-dimensional system, with nodes and antinodes at successive molecules of type $X$. If the coupling is in phase, maximum and minimum values of the orientational coordinate occur at the antinodes (right-hand side of the figure) so that the displacement wave is in phase with the orientational order parameter. If the coupling is out-of-phase, the preferred orientational order will have maximum and minimum values at the nodes of the displacement as in the left-hand side of the figure. Thus an instantaneous fluctuation in the phonon coordinate is accompanied by a fluctuation in the orientational order.

If $\mathbf{k}$ is in a general direction, there are no further symmetry requirements for translation-rotation coupling to

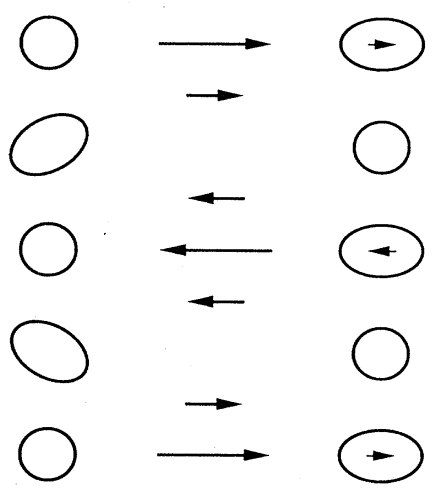

FIG. 6. Demonstration of in-phase coupling of an $L=1$ order parameter, such as a dipole (on the right), and the out-of-phase coupling of an $L=2$ order parameter, such as a quadrupole moment (on the left), with phonon displacements (center). 
exist, but in directions of higher symmetry (such as along $\langle 100\rangle$ in cubic crystals) and at special points, such as the $\Gamma$ point (zone center, $k=0$ ) and zone boundaries, there are more restrictions on coupling. As the order parameters for the ordered phases are commonly at points of higher symmetry, these extra restrictions are important and depend on the space group of the crystal structure concerned (Michel and Parlinski, 1985).

\section{E. Coupling at low $\mathbf{k}$ values}

The determination of the coupling between translational phonons and orientational variables at low $\mathbf{k}$ values is fairly straightforward and can be based on the $\Gamma$ point symmetry. Let us consider coupling terms of order $\mathbf{k}$ and $\mathbf{k}^{2}$.

It can be seen from Fig. 6 that out-of-phase coupling at long wavelengths occurs if the crystal field determining the preferred orientation at a particular site depends on the gradient of the displacement field, while in-phase coupling results when the local crystal field depends on the second derivative of the field. This result may also be found by performing a Taylor expansion of the coupling coefficients $v(\mathbf{k})$ about $\mathbf{k}=0$. Using the earlier result [Eq. (3.28)] that for rotator functions with $g$ (gerade) symmetry (those with even $L) v(\mathbf{k})$ is an odd function of $\mathbf{k}$ containing $\sin (\mathbf{k} \cdot \mathbf{X})$ terms, we obtain

$$
v_{\rho \mu}\left(k_{\alpha}\right)=i k_{\alpha} \frac{\partial v_{\rho \mu}(0)}{\partial k_{\alpha}}+\cdots \quad(L \text { even }),
$$

which is the expected coupling to the crystal-field gradient. The fact that $v$.is imaginary again implies that the coupled orientational variable is out-of-phase with the displacement wave. For odd $L$ variables that have $u$ (ungerade) symmetry, on the other hand,

$$
v_{\rho \mu}(\mathbf{k})=v_{\rho \mu}(0)-\frac{1}{2} k_{\alpha} k_{\beta} \frac{\partial^{2} v_{\rho \mu}(0)}{\partial k_{\alpha} \partial k_{\beta}}+\cdots(L \text { odd }) .
$$

\section{F. Coupling to acoustic phonons}

Acoustic displacement coordinates were defined in Eq. (3.15); from Eq. (3.21) we can write

$$
V_{a c}^{T R}=\widetilde{u}^{a c}(\mathbf{k})^{\dagger} \cdot v^{a c}(\mathbf{k}) \cdot \widetilde{\Delta}(\mathbf{k}),
$$

where the coupling constants in the matrix $v^{a c}$ are found from Eqs. (3.24) and (3.25) to be

$$
v_{i \mu}^{a c}=m^{-1 / 2} \sum_{\kappa}\left[\widetilde{c}_{i \kappa \mu}^{\prime}(\mathbf{k}=0)-\widetilde{c}_{i \kappa \mu}^{\prime}(\mathbf{k})\right] g_{L} g_{0}
$$

The sum over $\kappa$ is over different types of molecule and atomic ion in the crystal, and $m$ is the sum of the masses per unit cell. From this equation it can be seen that there is no coupling of orientational order to the acoustic phonons at the zone center, $\mathbf{k}=0$. The physical reason for this is that the $\mathbf{k}=0$ limit of an acoustic phonon is a uniform translation of the whole crystal lattice. At small but nonzero values of $k$ the coupling depends on the $L$ value of the interaction. From the argument in the previous section we see that it is linear in $\mathbf{k}$ for $L$ even, while for $L$ odd the lowest-order terms are quadratic in $\mathbf{k}$.

In the long wavelength limit we shall show (Sec. VI) that any terms that are linear in $k$ in the coupling of orientational waves to acoustic phonons lead to a coupling to lattice strains at $\mathbf{k}=0$ with a consequent softening of some of the elastic constants. The symmetry arguments just given show that only orientational variables with even values of $L$ can contribute to this.

\section{G. Coupling at larger $\mathbf{k}$ values}

Away from the neighborhood of the zone center the possibility or not of coupling depends on the direction of $\mathbf{k}$ relative to the crystal axes (or to the axes of the reciprocal lattice). If $\mathbf{k}$ lies in a general direction there are no symmetry restrictions on translation-rotation coupling, although coupling to $u$ type variables (odd $L$ ) will be in-phase and even in $\mathbf{k}$, while coupling to $g$ variables (even $L$ ) will be out-of-phase and odd in $\mathbf{k}$. However, if $\mathbf{k}$ is along a special direction of high symmetry, then there are symmetry restrictions on coupling, which may change at the zone boundary if the zone boundary point has higher symmetry. Translation-rotation coupling can only occur between displacement variables and rotator functions, which transform in the same way under the full space group.

The symmetry of wave functions in crystals with a definite $\mathbf{k}$ vector was first considered by Bouckaert et al. (1936). The examples that they used were cubic crystals. As many orientationally disordered crystals are cubic, we now consider these in greater detail. The axes of reciprocal space (containing the $\mathbf{k}$ vectors) are parallel to those of the real crystal. Directions of high symmetry occur if $\mathbf{k}$ is $[\xi 00]$ (the $\Delta$ directions), $[\xi \xi 0](\Sigma)$ or the $[\zeta \zeta \zeta]$ directions $(\Lambda)$. Note we use the notation that square brackets denote the star of equivalent $\mathbf{k}$ vectors. Table II shows the symmetries of collective variables in an fcc lattice with $F m \overline{3} m$ symmetry. In the column on the left-hand side are the various side point-group symmetry species (group $O_{h}$ ), together with symmetries of possible singlemolecule rotator functions or displacement variables. If one then constructs collective variables from linear combinations of these single-molecule functions with coefficients of the form $\exp [-i \mathbf{k} \cdot \mathbf{X}]$ as in Eq. (2.45), then these collective variables transform according to some symmetry species of the full space group. At the zone center $(\mathbf{k}=0)$ the relevant symmetry species are denoted by ${ }^{*} \Gamma$ with appropriate subscripts and superscripts. There is a one-to-one correspondence between the single-molecule symmetry species and the zone-center full group species. Away from the zone center the symmetry of the collective functions can be found from the compatibility tables (Boyle, 1990) for symmetries at 
TABLE II. Relation of symmetries of single molecules and collective variables in an fcc lattice.

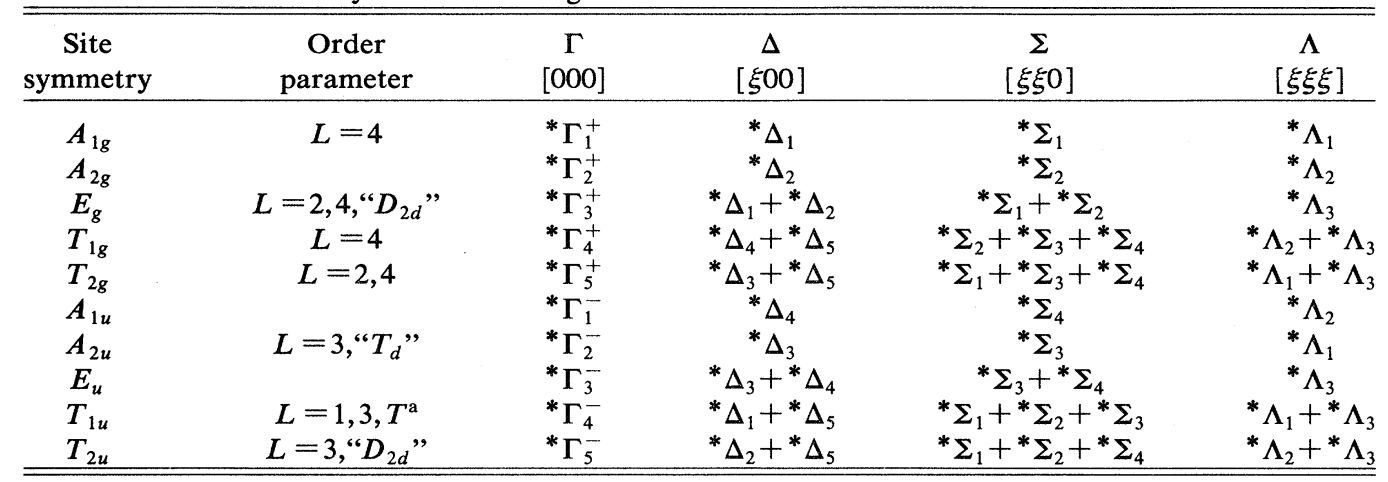

${ }^{\text {a }}$ Translational displacement of center of mass. LA phonons transform as $\Delta_{1} ; \Sigma_{1} ; \Lambda_{1}$.

different $\mathbf{k}$ values and are shown in Table II. To use this table one identifies the symmetry of the single-molecule displacement or orientational variable concerned in the site group (left-hand column) and then finds the symmetry of the collective variables for the value of $\mathbf{k}$ that one is interested in. If the phonon and orientational variables for this $\mathbf{k}$ value have the same symmetry, they may couple, otherwise not. For example, consider a collective variable made from $A_{2 u}$ orientational functions. At the zone center such a variable transforms as ${ }^{*} \Gamma_{2}^{-}$and does not couple to any displacements that transform as ${ }^{*} \Gamma_{4}^{-}$. However, if $\mathbf{k}$ is in a $\Sigma$ direction or in a $\Lambda$ direction, coupling to phonon modes is possible. One may also deduce whether a particular orientational variable couples to longitudinal or transverse phonons as the longitudinal phonon mode always transforms as the most symmetric irreducible representation of the space group. For example, it can be seen from Table II that in the $\Lambda$ direction a collective rotator function made from $L=3$ variables (which transform as $A_{2 u}$ in $O_{h}$ ) transforms as ${ }^{*} \Lambda_{1}$ and so couples with the longitudinal mode, but as the corresponding function in the $\Sigma$ direction transforms as ${ }^{*} \Sigma_{3}$ rather than ${ }^{*} \Sigma_{1}$, it couples with one of the transverse modes rather than with the longitudinal mode.

\section{H. Pseudospin description of translation-rotation coupling}

Translation-rotation coupling may also be formulated using the discrete or pseudospin variables $\widetilde{\Xi}(\mathbf{k})$ introduced in Sec. II.C. We consider as an example the orthorhombic disordered phase of sodium nitrite (Heine et al., 1984). Since the crystal field exerted by the surrounding $\mathrm{Na}^{+}$ions is strong, the molecular dipole moment of each nitrite molecule points in either the $+b$ or the $-b$ direction. The site symmetry is $D_{2 h}$. In analogy with the discussion in Sec. II.E we define variables $\xi_{+}$and $\xi_{-}$, which have values 1 or 0 according to the direction of the nitrite dipole. Symmetry-adapted discrete variables, $\Xi_{A_{1 g}}=\xi_{+}+\xi_{-}$and $\Xi_{B_{1 u}}=\xi_{+}-\xi_{-}$, can be formed in exactly the same way as described in Sec. II.C for ammonium bromide. The variable $\Xi_{B_{1 u}}$ has values \pm 1 depending on which way the molecule is oriented and behaves like a spin variable. We can form collective variables, $\widetilde{\Xi}_{B_{1 u}}$, from these [cf. Eq. (2.57)], and write the translationrotation coupling as

$$
V^{T R}=\sum_{\mathbf{k}} \tilde{u}^{\dagger}(\mathbf{k}) \cdot v(\mathbf{k}) \cdot \widetilde{\Xi}(\mathbf{k})
$$

where $\widetilde{\Xi}$ is a column vector containing in this example the single element $\Xi_{B_{1 u}}(\mathbf{k}) ; u$ is a row vector containing six elements, the $x, y$, and $z$ displacements of $\mathrm{NO}_{2}^{-}$and $\mathrm{Na}^{+}$ions, respectively.

To determine the values of $v(\mathbf{k})$ in terms of intermolecular interactions, it is necessary to follow the same arguments in terms of orientational variables as in the previous section (III.C). We can restrict ourselves to displacements of the center of mass of the molecules and of the sodium counterions in the $y$ direction, parallel to the b axis. In the linear approximation we again consider only the orientation of a particular nitrite $n$ and average all other nitrite ions over their two configurational states. When this averaging has been performed, the energy of a molecule in an ideal lattice does not depend on whether the molecule is facing in the $\pm \mathbf{b}$ direction, that is, which of the discrete variables $\xi_{+}$or $\xi_{-}$is equal to one, but the change in its energy with displacement does depend on the direction in which it is oriented. The energy $V^{+}$of a molecule facing in the $+b$ direction is, to first order in the lattice displacements,

$$
V_{n}^{+}=V_{0}+\sum_{i} \sum_{n^{\prime}} \frac{\partial V^{+}\left(\mathbf{X}_{n n^{\prime}}\right)}{\partial X_{i n n^{\prime}}}\left[u_{i}(n)-u_{i}\left(n^{\prime}\right)\right]+\cdots
$$

If it is facing in the other direction, a similar equation with $V^{-}$for $V^{+}$obtains, so that we can write

$$
V_{n}=\sum_{n^{\prime}} \sum_{i} c_{i}^{\prime}\left(\mathbf{X}_{n n^{\prime}}\right) \Xi_{B_{1 u}}\left[u_{i}(n)-u_{i}\left(n^{\prime}\right)\right]
$$

This equation has exactly the same form as Eq. (3.19), which we derived for the rotator function treatment, if we replace the rotator function $\Delta$ by the pseudospin vari- 
able $\Xi$, and set all form factors equal to 1 . In the pseudospin treatment the coefficients $c^{\prime}$ are determined from sums rather than integrals. In this example

$$
c_{i}^{\prime}\left(X_{n n^{\prime}}\right)=\frac{1}{2}\left[\frac{\partial V^{+}}{\partial X_{i n n^{\prime}}}-\frac{\partial V^{-}}{\partial X_{i n n^{\prime}}}\right]
$$

The coupling matrix elements $v(\mathbf{k})$ are determined from the $c^{\prime}$ by Eqs. (3.23)-(3.25). The matrix $v$ in this example contains only two terms, a coupling of the $B_{1 u}$ pseudospin variable to both the nitrite displacements in the $y$ direction and the sodium $y$ displacements. As the pseudospin variable is odd under inversion, the coupling of orientational order and phonon displacement is in phase, and the exp term in the definition of $\widetilde{c}^{\prime}$ [Eq. (3.22)] may be replaced by a cosine. The matrix elements are for the nitrite displacements

$$
\begin{aligned}
v_{\rho B_{1 u}}(\mathbf{k})=\frac{1}{m_{\mathrm{NO}_{2}}^{1 / 2}}\left\{\sum_{n^{\prime}=\mathrm{NO}_{2}} c_{y}^{\prime}\left(\mathbf{X}_{n n^{\prime}}\right)\left[1-\cos \mathbf{k} \cdot \mathbf{X}_{\mathrm{nn}}\right]\right. \\
\left.\quad+\sum_{n^{\prime}=\mathrm{Na}} c_{y}^{\prime}\left(\mathbf{X}_{n n^{\prime}}\right)\right\}
\end{aligned}
$$

and for the sodium ion displacements

$$
v_{\rho B_{1 u}}(\mathbf{k})=-\frac{1}{m_{\mathrm{Na}}^{1 / 2}} \sum_{n^{\prime}=\mathrm{Na}} c_{y}^{\prime}\left(\mathbf{X}_{n n^{\prime}}\right) \cos \mathbf{k} \cdot \mathbf{X}_{\mathrm{nn}^{\prime}}
$$

These expressions are more general than previous results (Michel, 1981) as they take into account the interaction of a central nitrite molecule at site $(n)$ with all the ions in the crystal, sodium and nitrite, rather than just the surrounding sodium ions.

Coupling to the acoustic phonons is just

$$
v_{y B_{1 u}}^{a c}=m^{-1 / 2} \sum_{n^{\prime}} c_{y}^{\prime}\left(\mathbf{X}_{n n^{\prime}}\right)\left[\cos \mathbf{k} \cdot \mathbf{X}_{n n^{\prime}}-1\right],
$$

which in the long wavelength limit reduces to

$$
v_{y B_{1 u}}^{a c}=\frac{1}{2 m^{-1 / 2}} \sum_{n^{\prime}} c_{y}^{\prime}\left(\mathbf{X}_{n n^{\prime}}\right)\left(\mathbf{k} \cdot \mathbf{X}_{n n^{\prime}}\right)^{2} .
$$

The fact that this is of order $k^{2}$ rather than $k$ means that the translation-rotation coupling does not facilitate the zone-center phase transition to the ferroelectric phase. It is sufficiently strong, however, to cause a transition from the disordered phase to an incommensurate phase with a finite but small value of $\mathbf{k}$ that is stable for a few degrees above the ferroelectric phase (Heine and McConnell, 1981; Heine et al., 1984; Lynden-Bell et al., 1984).

Since $\mathrm{NaNO}_{2}$ contains only two ions per primitive unit cell, it is instructive to consider the coupling of optical phonons to the pseudospin states at the zone center (Ehrhardt and Michel, 1981a, 1981b). Restricting ourselves again to displacements along the $b$ axis, the optical basis vector $w_{2}$ [compare with Eq. (3.14) for the acoustic basis vector] has two nonzero components

$$
w_{y}^{\text {opt }}=\left[0,\left(\frac{\mu}{m_{1}}\right]^{1 / 2}, 0,0,-\left[\frac{\mu}{m_{2}}\right]^{1 / 2}, 0\right] \text {, }
$$

where $\mu=m_{1} m_{2} / m$ is the reduced mass. The optical displacements are given by

$$
\widetilde{u}_{y}^{\mathrm{opt}}(\mathbf{k})=\sum_{\rho} w_{y \rho}^{\mathrm{opt}} \widetilde{u}_{\rho}(\mathbf{k}),
$$

with $\rho=\left(\mathrm{NO}_{2}^{-}, y\right)$ and $\left(\mathrm{Na}^{+}, y\right)$. The coupling with $\mathrm{NO}_{2}^{-}$ orientations then reads

$$
V^{T R}=\sum_{\mathbf{k}} \tilde{u}_{\mathrm{opt}, y}^{\dagger}(\mathbf{k}) \cdot v_{y B_{1 u}}^{\mathrm{opt}} \cdot \widetilde{\Xi}_{B_{1}}(\mathbf{k})
$$

with

$$
v_{y B_{1}}^{\mathrm{opt}}=\sum_{\rho} w_{\rho}^{\mathrm{opt}} v_{\rho B_{1 u}}(\mathbf{k}) .
$$

In particular, at the zone center we obtain by substitution in Eqs. (3.37) and (3.38)

$v_{y B_{1 u}}^{\mathrm{opt}}(0)=\mu^{1 / 2}\left[\frac{1}{m_{\mathrm{Na}}}-\frac{1}{m_{\mathrm{NO}_{2}}}\right] \sum_{n^{\prime}=\mathrm{Na}} c_{y}^{\prime}\left(\mathbf{X}_{n n^{\prime}}\right)$.

We see that only counterions contribute to the translation-rotation coupling at $\mathbf{k}=0$. This is understandable since all the nitrites move in phase at $\mathbf{k}=0$ and therefore do not influence their relative orientations.

\section{Single-molecule description of translation-rotation coupling}

In the last few sections we have concentrated on a collective description of the translation-rotation coupling process. This is the most appropriate description for phenomena that depend on the crystal as a whole, such as elastic constants, phase transitions, and phonon spectra. However, the physical interactions causing the translation-rotation coupling are local, and it is instructive at this point to return to a molecule-based description in order to discuss the effects of symmetry on the translation-rotation coupling at a particular site. Most experimental methods probe collective rather than local (single-molecule) properties, but there are some, such as NMR, which measure averages of local properties. In computer simulations one can measure the details of the dynamics of a single molecule as well as averages of both single-molecule and collective properties.

The translation-rotation coupling at a particular molecule $n$ is given by Eq. (3.19), which reads

$$
V^{T R}=\sum_{n, n^{\prime}} \sum_{\mu, i} c_{i \mu}^{\prime}\left(\mathbf{X}_{n n^{\prime}}\right) \Delta_{\mu}(n) g_{L} g_{0}\left[u_{i}(n)-u_{i}\left(n^{\prime}\right)\right]
$$

If the molecule $n$ is at a site with a center of symmetry, then there are molecules at both $+\mathbf{X}$ and $-\mathbf{X}$ that interact in the same way. It has already been argued in Sec. III.D that molecule $n^{\prime}$ and its inverse must move by equal amounts in opposite directions to preserve the center of symmetry of the site. If they move by equal amounts in the same direction the change in potential is antisymmetric under the inversion of the site coordi- 
nates. As $\Delta$ functions with even values of $L$ are even, and those of odd values of $L$ are odd under this operation, we see that a single-molecule rotator function with an even- $L$ value couples with a local distortion that has a node at the molecule concerned, while rotator functions with odd- $L$ values couple with local distortions whose maxima are at the molecule concerned. This behavior is clear in Fig. 6 when viewed from a single-molecule point of view and results in the fact that even- $L$ rotator functions of a particular molecule never couple to the displacement of the same molecule. The argument may be extended as, at least for small displacements, the displacement field at a molecule transforms as $L=1$, its gradient as $L=2$, and its second derivative as $L=3$, and these terms can only couple to the rotator functions of the same $L$ values.

\section{J. Form of the potential energy}

The result of this section can be summarized by saying that the potential energy can be written

$$
V=V^{T T}+V^{R}+V^{T R}+V^{R R}+V^{R R T} \cdots,
$$

where $V^{T T}$ describes the center-of-mass vibrational potential energy, $V^{R}$ describes the crystal field potential felt by one molecule in a rigid ideal lattice with the orientations of all other molecules averaged over their distribution functions, $V^{T R}$ is the translation-rotation coupling describing the interaction between orientations and displacements, and $V^{R R}$ describes the direct interaction between the orientations of pairs of molecules, while the final term is a trilinear term in orientations and displacements. This expansion can be extended to include higher-order coupling terms if required. In the harmonic or renormalized harmonic approximation (see, e.g., Horner, 1974) the phonon term $V^{T T}$ is

$$
V^{T T}=\sum_{\mathbf{k}} \frac{1}{2} \widetilde{u}(\mathbf{k})^{\dagger} \cdot M(\mathbf{k}) \cdot \widetilde{u}(\mathbf{k}),
$$

where $M$ is the dynamical matrix describing the phonon frequencies in the absence of translation-rotation coupling (the bare frequencies).

The total expression for the potential energy to second order can be written

$$
V=V^{R}+\sum_{\mathbf{k}} \frac{1}{2}\left[\tilde{u} \cdot{ }^{\dagger} M \cdot \widetilde{u}+\widetilde{u} \cdot{ }^{\dagger} v \cdot \widetilde{\Delta}+\widetilde{\Delta}^{\dagger} \cdot v^{\dagger} \cdot \widetilde{u}+\widetilde{\Delta}^{\dagger} \cdot J \cdot \widetilde{\Delta}\right],
$$

where the sum is over all $\mathbf{k}$ values and, to simplify the notation, we have omitted the explicit $\mathbf{k}$ dependence of all the terms in the square brackets. The values of the matrices $M, v$, and $J$ depend on the details of the intermolecular forces in the crystal, and, if these are known, the matrices $v$ and $J$ can be calculated using the techniques explained in this section. In the following section we will use the potential $V$ [Eq. (3.49)] as a starting point for a derivation of the free energy and other thermodynamic quantities that describe the thermoelastic behavior of orientationally disordered crystals.

\section{FREE ENERGIES AND SUSCEPTIBILITIES}

\section{A. The Landau free energy}

The most important thermodynamic quantity for a system is its free energy. It can be used to determine other macroscopic thermodynamic properties and to investigate phase transitions as these occur when two phases with different structures attain the same free energy. The Helmholtz free energy $A$ is the free energy of a system at equilibrium and is related to the partition function of the system in a canonical ensemble. This corresponds to macroscopic thermodynamic conditions of constant volume and temperature $T$. The Landau free energy $F$ is the free energy of the system when it is constrained so that the mean value of some order parameter $Y$ is equal to $\bar{Y}$. The Landau theory of phase transitions, which can be applied to the order-disorder phase transitions in orientationally disordered crystals, is based on an expansion of the free energy $F$ in a power series in order parameters (Landau, 1937; Landau and Lifschitz, 1980)

$$
F=F_{0}+A \bar{Y}^{2}+B \bar{Y}^{3}+C \bar{Y}^{4} .
$$

The actual value of $\bar{Y}$ in an unconstrained system is the value for which $F$ is a minimum. In a disordered crystal all other parameters are equal to zero, so that the condition for such a phase to be stable is that $F$ is a minimum at $\bar{Y}=0$. In the expression (4.1) this is true if the coefficient $A$ is greater than or equal to zero. If the coefficient $B$ is identically zero and $C>0$, the Landau expansion leads to a second-order phase transition at a temperature $T_{c}$ where $A$ changes sign. On the other hand, if $B$ is still zero but $C<0$, the phase transition is of first order and the expansion has to be extended to sixth-order terms. If $B \neq 0$, the phase transition is always of first order. Whether $B$ is different from zero or not depends on the symmetry of the order parameter. When $B=0$, the magnitude and sign of $C$ can sometimes be tuned by external pressure. This effect is due to a coupling that is quadratic in the order-parameter fluctuations and linear in the lattice displacements, i.e., terms of the form $V^{R R T}$ in the potential energy. These terms give a negative contribution to the coefficient $C$. The mechanism is well known in compressible magnetic lattices (Wagner and Swift, 1970; Bergman and Halperin, 1976) and is also relevant in molecular crystals (Michel and Theuns, 1989). Here we shall restrict ourselves to a consideration of the bilinear coupling $V^{T R}$, which affects the value of the coefficient $A$ in the Landau expansion. Indeed, if this coupling is sufficiently strong, it is dominant in driving the structural phase transition irrespective of whether it is first or second order. In orientationally disordered crystals there are many possible order parameters, and the Landau free energy is written as a power series in all 
the order parameters and includes cross terms between them. Each term must be totally symmetric under the symmetry operations of the crystal space group. It is convenient to use the mean values of the phonon displacement coordinates, $\bar{u}(\mathbf{k})=\langle\tilde{u}(\mathbf{k})\rangle$, and the mean values of the rotator coordinates, $\bar{\Delta}(\mathbf{k})=\langle\widetilde{\Delta}(\mathbf{k})\rangle$, as order parameters. We shall show that, in a mean-field approximation,

$$
\begin{array}{r}
F=F_{0}+\sum_{\mathbf{k}} \frac{1}{2}\left[\bar{u}^{\dagger} \cdot M \cdot \bar{u}+\bar{u}^{\dagger} \cdot v \cdot \bar{\Delta}+\bar{\Delta}^{\dagger} \cdot v^{\dagger} \cdot \bar{u}\right. \\
\left.+\bar{\Delta}^{\dagger} \cdot\left(\chi_{0}^{-1}+J+j\right) \cdot \bar{\Delta}\right]+\cdots,
\end{array}
$$

where $M$ and $v$ are the $\mathbf{k}$-dependent coupling-constant matrices appearing in the Hamiltonian [Eq. (3.49)], $j$ is a self-energy matrix that is independent of $\mathbf{k}$, and $\chi_{0}$ is a single-particle susceptibility matrix. The criterion for stability of the disordered phase is that $F$ is a minimum when all the order parameters are zero. This can be found by imposing the condition $(\partial F / \partial \bar{u})_{\bar{\Delta}, T, V}=0$ and eliminating $\bar{u}$ from the equation for the Landau free energy to give

$$
F=F_{0}+\sum_{\mathbf{k}} \frac{1}{2} \bar{\Delta}^{\dagger} \cdot\left(\chi_{0}^{-1}+J+j-v^{\dagger} \cdot M^{-1} \cdot v\right) \cdot \bar{\Delta}
$$

which shows that the disordered phase is stable provided that all the eigenvalues of the matrix $\left(\chi_{0}^{-1}+J\right.$ $\left.+j-v^{\dagger} M^{-1} v\right)$ for each $\mathbf{k}$ value are greater than zero. We see from this that the effect of the translationrotation coupling term $v$ is to replace the coupling matrix $J$ by an effective coupling

$$
J^{\prime}=\left(J+j-v^{\dagger} \cdot M^{-1} \cdot v\right) .
$$

Thus translation-rotation coupling provides an indirect mechanism for coupling between the orientational order parameters that acts in addition to the direct coupling and may even be the dominant coupling term. The physical origin of this effect is the enhancement of orientational order in a lattice with a permanent distortion $\bar{u}$ and is similar to the time-dependent ordering associated with fluctuations in the phonon displacements discussed in Sec. III. Instability sets in when $J^{\prime}$ (or its largest eigenvalue) is more negative than $\chi_{0}^{-1}$. While the direct coupling $J$ may have either sign, $j$ is always positive, and the indirect term $v^{\dagger} \cdot M^{-1} \cdot v$ always reduces $J^{\prime}$, increasing the tendency for instability.

In many respects the bilinear coupling of orientational modes to lattice deformations in molecular crystals is analogous to the coupling of electronic degrees of freedom of Jahn-Teller ions to lattice deformations (Kanamori, 1960; Elliott et al., 1972; Gehring and Gehring, 1975). In general, phase transitions where an order parameter is coupled to strain components are known as ferroelastic transitions. Their symmetry properties have been extensively discussed by Toledano and Toledano (1980).

Expressions for the Landau free energy have been de- rived previously for an orientationally disordered crystal with translation-rotation coupling (Michel and Courtens, 1981; Michel and Parlinski, 1985) using a Lagrange multiplier method. That procedure was inspired from earlier work of Feder and Pytte (1973) on Jahn-Teller phonon coupled systems. Here we will present an alternative derivation of the free energy based on a variational method. The final result of these two methods is the same and gives an expression for the free energy as a functional of the displacive and orientational variables. Other derivations of expressions for the free energy include that of Sahu and Mahanti (1984), who started from an effective rotational Hamiltonian in which the linear coupling to the phonon variables had been removed by a canonical transformation, and that of De Raedt et al. (1981) from a series expansion of the partition function. Earlier, Press and Hüller (1973b) derived an expression for the Landau free energy of $\mathrm{CD}_{4}$ on a rigid lattice.

\section{B. Calculation of the Helmholtz free energy and the susceptibilities from the microscopic Hamiltonian}

The susceptibilities of the system provide a convenient route for connecting the microscopic Hamiltonian with the macroscopic Landau free energy. Susceptibilities are also important as they can be measured from equilibrium fluctuations either in computer simulations or by experimental techniques such as diffuse $x$-ray and neutron scattering.

Suppose that the system has a pair of order parameters $\bar{u}$ and $\bar{\Delta}$. To determine the susceptibilities of the system, we find the equilibrium Helmholtz free energy $A\left(G_{u}, G_{\Delta}\right)$ in the presence of fictitious fields $G_{u}$ and $G_{\Delta}$, which interact with the phonon and rotator order parameters, respectively. The resulting Hamiltonian is

$$
H=T+V+\sum_{\mathbf{k}} \tilde{u}^{\dagger} \cdot G_{u}+\sum_{\mathbf{k}} \widetilde{\Delta}^{\dagger} \cdot G_{\Delta},
$$

where $V$, Eq. (3.49), is the potential energy of the crystal in the absence of the fields and $T$ is the kinetic energy. $G_{u}(\mathbf{k})$ and $G_{\Delta}(\mathbf{k})$ are vectors in the same space as $u$ and $\Delta$, respectively.

The Helmholtz free energy of a classical system is related to the Hamiltonian through the canonical partition function, which has momentum- and position-dependent parts. All position-dependent properties are determined by the configuration integral $Z$, defined by an integral over all position coordinates

$$
Z=\int \exp \left(-V_{\text {tot }} / k_{B} T\right) d^{N} \mathbf{r},
$$

where $V_{\text {tot }}$ is the potential energy term in the Hamiltonian, including any field-dependent terms. In particular, the configuration part of the Helmholtz free energy is given by (Landau and Lifschitz, 1980).

$$
A=-k_{B} T \log Z \text {. }
$$

The average value of any position-dependent quantity $q$ is 
given by

$$
\bar{q}=Z^{-1} \int q \exp \left(-V_{\text {tot }} / k_{B} T\right) d^{N} \mathbf{r} .
$$

From Eqs. (4.5) to (4.8) it can be seen that differentiating $A$ with respect to the fields gives the order parameters, so that differentiating twice gives susceptibilities; thus,

$$
\begin{aligned}
& \frac{\partial A}{\partial G_{u}(-\mathbf{k})}=\bar{u}(\mathbf{k}), \quad \frac{\partial A}{\partial G_{\Delta}(-\mathbf{k})}=\bar{\Delta}(\mathbf{k}),-\frac{\partial^{2} A}{\partial G_{u}(\mathbf{k}) \partial G_{u}(-\mathbf{k})}=\chi_{u u}=\langle\widetilde{u}(\mathbf{k}) \tilde{u}(-\mathbf{k})\rangle / k_{B} T \\
& -\frac{\partial^{2} A}{\partial G_{u}(\mathbf{k}) \partial G_{\Delta}(-\mathbf{k})}=\chi_{u \Delta}=\langle\widetilde{u}(-\mathbf{k}) \widetilde{\Delta}(\mathbf{k})\rangle / k_{B} T,-\frac{\partial^{2} A}{\partial G_{\Delta}(\mathbf{k}) \partial G_{\Delta}(-\mathbf{k})}=\chi_{\Delta \Delta}=\langle\widetilde{\Delta}(\mathbf{k}) \widetilde{\Delta}(-\mathbf{k})\rangle / k_{B} T .
\end{aligned}
$$

In order to evaluate $A\left(G_{u}, G_{\Delta}\right)$, we use the Hamiltonian of Eq. (4.5), which includes the fields to obtain

$$
Z=\int \exp -\left[V^{R}+\sum_{\mathbf{k}} \frac{1}{2}\left[\widetilde{u}^{\dagger} \cdot M \cdot \widetilde{u}+\tilde{u}^{\dagger} \cdot v \cdot \Delta+\widetilde{\Delta}^{\dagger} \cdot v^{\dagger} \cdot \widetilde{u}+\widetilde{\Delta}^{\dagger} \cdot J \cdot \widetilde{\Delta}\right]+G_{u}^{\dagger} \cdot \widetilde{u}+G_{\Delta}^{\dagger} \cdot \widetilde{\Delta}\right] / k_{B} T d^{N} \mathbf{r}
$$

We now eliminate the cross terms between phonon and orientational variables by defining a set of modified phonon variables forming a column vector $\widetilde{U}$ with k-dependent components

$$
\widetilde{U}=\tilde{u}+M^{-1} \cdot v \cdot \widetilde{\Delta}+M^{-1} \cdot G_{u}
$$

Note that the quantities $M, v$, and $G_{u}$, which appear in this equation, are $\mathbf{k}$ dependent. Substituting this in Eq. (4.10) gives

$$
\begin{aligned}
Z=-\int \exp \sum_{\mathbf{k}} \frac{U^{\dagger} \cdot M \cdot U}{2 k_{B} T} \exp \sum_{\mathbf{k}} \frac{G_{u}^{\dagger} \cdot M^{-1} \cdot G_{u}}{2 k_{B} T} \\
\quad \times \exp -\left\{V^{R}+\frac{1}{2} \sum_{\mathbf{k}}\left[\widetilde{\Delta}^{\dagger} \cdot\left(J-v^{\dagger} M^{-1} v\right) \cdot \widetilde{\Delta}+\left(G_{\Delta}^{\dagger}-G_{u}^{\dagger} \cdot M^{-1} \cdot v\right) \cdot \widetilde{\Delta}\right]\right\} / k_{B} T d^{N} \mathbf{r} .
\end{aligned}
$$

The integration over the $3 N$ coordinates can be resolved into integrations over the center-of-mass positions and over molecular orientations. Integrating over the center-of-mass coordinates is equivalent to integrating over the modified phonon variables and results in the replacement of the first exponential term in the integral by products of terms of the form $\left[2 \pi k_{B} T / M(\mathbf{k})\right]^{1 / 2}$, which are the same as one would obtain from the phonon term in the absence of translationrotation coupling. Thus the Helmholtz free energy is the sum of an unmodified phonon term, a field-dependent term and a rotational term

$$
A=A_{\mathrm{ph}}^{0}-\frac{1}{2} \sum_{\mathbf{k}} G_{u}^{\dagger} \cdot M^{-1} \cdot G_{u}-k_{B} T \log Z_{\mathrm{rot}}
$$

The rotational partition function is

$$
Z_{\text {rot }}=\int \exp -\left\{V^{R}+\sum_{\mathbf{k}}\left[\frac{1}{2} \widetilde{\Delta}^{\dagger} \cdot\left(J-v^{\dagger} M^{-1} v\right) \cdot \widetilde{\Delta}+G_{1}^{\dagger} \cdot \widetilde{\Delta}\right]\right\} / k_{B} T d^{N} \omega,
$$

where $G_{1}$ is a new field defined as

$$
G_{1}=G_{\Delta}-v^{\dagger} \cdot M^{-1} \cdot G_{u}
$$

To evaluate $Z_{\text {rot }}$ one must evaluate Eq. (4.14) by integrating over the orientation of each molecule.

\section{Mean-field approximation}

This rotational configuration integral cannot readily be evaluated, and we seek an approximation to $A$. We shall use a variational method equivalent to the mean-field approximation. First, we take the self-interaction, which is really a single-particle term (cf. the discussion in Sec. III.A), out of the term that is quadratic in the rotator functions and put it into a new effective single-particle potential $W^{R}$ 


$$
\begin{aligned}
W^{R} & =V^{R}-\frac{1}{2} \Delta\left(\omega_{n}\right)^{\dagger} \cdot\left\langle v^{\dagger} M^{-1} v\right\rangle_{\mathrm{k}} \cdot \Delta\left(\omega_{n}\right) \\
& =V^{R}-\frac{1}{2} \Delta^{\dagger} \cdot j \cdot \Delta
\end{aligned}
$$

where $j$ is a self-coupling matrix that is the average of the indirect-coupling matrix $v^{\dagger} \cdot M^{-1} \cdot v$ over all $3 N \mathbf{k}$ values

$$
j=\frac{1}{3 N} \sum_{\mathbf{k}} v^{\dagger} \cdot M^{-1} \cdot v
$$

This gives

$$
Z_{\mathrm{rot}}=\int \exp -\left[W^{R}+\sum_{\mathbf{k}}\left(\frac{1}{2} \tilde{\Delta}^{\dagger} \cdot J^{\prime} \cdot \widetilde{\Delta}+G_{1}^{\dagger} \cdot \widetilde{\Delta}\right)\right] / k_{B} T d^{N} \omega
$$

where the effective orientational coupling matrix $J^{\prime}$ is given by Eq. (4.4) and contains the direct coupling, the self-coupling, and the indirect contribution.

The rotational free energy is then estimated using the Bogoliubov inequality

$$
A_{\text {rot }} \leq A_{\text {trial }}+\left\langle H_{\text {rot }}-H_{\text {trial }}\right\rangle_{\text {trial }}
$$

with a trial Hamiltonian $H_{\text {trial }}=W^{R}+\Sigma_{\mathrm{k}} G_{2}^{\dagger} \cdot \widetilde{\Delta}$, where $G_{2}(\mathbf{k})$ are variational parameters to be determined. Expanding the configuration integral, we obtain (to quadratic order in $G_{2}$ )

$$
Z_{\text {trial }}=Z_{\text {rot }}^{0}\left[1-\sum_{\mathbf{k}} \frac{G_{2}^{\dagger}\langle\widetilde{\Delta}\rangle_{0}}{k_{B} T}+\sum_{\mathbf{k}} \frac{\left(G_{2}\right)^{2}\left\langle\widetilde{\Delta}^{2}\right\rangle_{0}}{2\left(k_{B} T\right)^{2}} \cdots\right],
$$

where

$$
Z_{\text {rot }}^{0}=\int \exp -\left[W^{R} / k_{B} T\right] d^{N} \omega .
$$

The difference in Hamiltonians is

$$
H_{\text {rot }}-H_{\text {trial }}=\sum_{\mathbf{k}}\left\{\frac{1}{2} \widetilde{\Delta} \cdot J^{\prime} \cdot \widetilde{\Delta}+\left(G_{1}^{\dagger}-G_{2}^{\dagger}\right) \cdot \widetilde{\Delta}\right\}
$$

while the value of $A_{\text {trial }}$ is

$$
A_{\text {trial }}=A_{\text {rot }}^{0}-\sum_{\mathbf{k}} \frac{1}{2} G_{2}^{\dagger} \cdot \chi_{0} \cdot G_{2}
$$

where $A_{\text {rot }}^{0}=-k_{B} T \log Z_{\text {rot }}^{0}$, and $\chi_{0}$ is the susceptibility matrix of the $\widetilde{\Delta}$ rotator functions calculated with the single-particle effective Hamiltonian $W^{R}$. The average values of the rotator functions are found by differentiating Eq. (4.23) with respect to $G_{2}$

$$
\bar{\Delta}=\frac{\partial A_{\text {trial }}}{\partial G_{2}}=-\chi_{0} \cdot G_{2},
$$

which can be used to compute the second term in the Bogoliubov inequality (4.19) to give

$$
A_{\text {rot }} \leq A_{\text {rot }}^{0}+\sum_{\mathbf{k}}\left\{\frac{1}{2} G_{2}^{\dagger} \cdot\left(\chi_{0}+\chi_{0} \cdot J^{\prime} \cdot \chi_{0}\right) \cdot G_{2}-G_{1}^{\dagger} \cdot \chi_{0} \cdot G_{2}\right\}
$$

Minimizing this with respect to the variational parameter $G_{2}$ gives

$$
A_{\text {rot }} \approx-\sum_{\mathbf{k}}\left\{\frac{1}{2} G_{1}^{\dagger} \cdot \chi_{0} \cdot\left[1+J^{\prime} \chi_{0}\right]^{-1} \cdot G_{1}\right\}
$$

so that our estimate of the total free energy is

$$
\begin{aligned}
A=A^{0}-\sum_{\mathbf{k}}\{ & \frac{1}{2} G_{u}^{\dagger} \cdot M^{-1} \cdot G_{u} \\
& \left.-\frac{1}{2} G_{1}^{\dagger} \cdot \chi_{0} \cdot\left[1+J^{\prime} \cdot \chi_{0}\right]^{-1} \cdot G_{1}\right\} .
\end{aligned}
$$

$\bar{\Delta}(\mathbf{k})$ and $\bar{u}(\mathbf{k})$ are determined from this expression by differentiation with respect to $G_{\Delta}(\mathbf{k})$ and $G_{u}(\mathbf{k})$ at fixed $\mathbf{k}$ using Eq. (4.15) to relate $G_{1}$ to these two fields. This gives

$$
\begin{aligned}
& \bar{\Delta}(\mathbf{k})=\frac{\partial A}{\partial G_{\Delta}(\mathbf{k})}=-\chi_{0}\left[1+J^{\prime} \chi_{0}\right]^{-1} G_{1}, \\
& \bar{u}(\mathbf{k})=\frac{\partial A}{\partial G_{u}(\mathbf{k})}=-M^{-1} \cdot G_{u}-M^{-1} \cdot v \cdot \bar{\Delta},
\end{aligned}
$$

where the k-dependent quantities $J^{\prime}, v, M, G_{1}$, and $G_{u}$ are evaluated at the chosen value of $\mathbf{k}$. The $\mathbf{k}$-dependent collective susceptibilities are determined from the second derivatives of $A$ with respect to the fields [Eqs. (4.9)] to be

$$
\begin{aligned}
\chi_{\Delta \Delta} & =\chi_{0} \cdot\left[1+J^{\prime} \cdot \chi_{0}\right]^{-1} \\
\chi_{\Delta u} & =-\left[1+J^{\prime} \chi_{0}\right]^{-1} \cdot v^{\dagger} \cdot M^{-1}, \\
\chi_{u u} & =M^{-1}+M^{-1} \cdot v \cdot \chi_{0}\left[1+\chi_{0} \cdot J^{\prime}\right]^{-1} \cdot v^{\dagger} \cdot M^{-1} \\
& =M^{-1}+M^{-1} \cdot v \cdot \chi_{\Delta \Delta} \cdot v^{\dagger} \cdot M^{-1} .
\end{aligned}
$$

It should be noted that there are no cross terms in susceptibilities between functions of different symmetry. In this mean-field approximation the orientational susceptibilities are well behaved unless $\left(\chi_{0}^{-1}+J^{\prime}\right)$ has a zero eigenvalue, the same criterion for the stability of the disordered phase that we have already determined from the Landau free energy (Sec. IV.A). It includes the effect of translation-rotation terms in the effective coupling constant $J^{\prime}$. When the orientational susceptibility diverges at some value of $\mathbf{k}$, Eqs. (4.29)-(4.31) show that $\chi_{u u}$ and $\chi_{u \Delta}$ diverge at the same value of $\mathbf{k}$. 
Susceptibilities and mean-square fluctuations are a useful way of finding precursor effects of the ordering phase transition in computer simulations. One may look for an anomalous temperature dependence or an abnormally large susceptibility, but a particularly useful quantity that is easy to compute is the correlation coefficient of fluctuations in phonon and rotator variables

$$
C_{\kappa \mu}=\frac{\left\langle\tilde{u}_{\kappa} \widetilde{\Delta}_{\mu}\right\rangle}{\left(\left\langle\tilde{u}_{\kappa}^{\dagger} \tilde{u}_{\kappa}\right\rangle\left\langle\widetilde{\Delta}_{\mu}^{\dagger} \widetilde{\Delta}_{\mu}\right\rangle\right)^{1 / 2}}
$$

If there is only one rotator variable and one phonon coordinate involved in the phase transition, then Eqs. (4.29)-(4.31) can be used to show that the modulus of this correlation coefficient is equal to unity at the phase transition. The determination of the positions of the maxima and minima of this function in $\mathbf{k}$ space and the size of the correlation coefficients at these points compared to unity have proved to be useful diagnostics of incipient ordering (Lynden-Bell et al., 1983, 1989; Dove and Lynden-Bell, 1986).

To obtain the Landau free energy $F$ from the Helmholtz free energy $A$, one uses the fact that $A$ can be written as a power series in the fields, $G_{u}$ and $G_{\Delta}$, together with the thermodynamic relation

$$
d A=-S d T-p d V+\sum_{\mathbf{k}}\left(\bar{u} d G_{u}+\bar{\Delta} d G_{\Delta}\right) .
$$

$F$ is a function of the order parameters and has the property that it is a minimum as a function of changes in order parameter at constant temperature and volume. Consider the combination $\left[A-\Sigma_{\mathbf{k}}\left(G_{u}^{\dagger} \bar{u}+G_{\Delta}^{\dagger} \bar{\Delta}\right)\right]$, whose differential is given by

$$
\begin{aligned}
d\left[A-\sum_{\mathbf{k}}\left(G_{u}^{\dagger} \bar{u}+G_{\Delta}^{\dagger} \bar{\Delta}\right)\right]= & -S d T-p d V \\
& -\sum_{\mathbf{k}}\left(G_{u}^{\dagger} d \bar{u}+G_{\Delta}^{\dagger} d \bar{\Delta}\right) .
\end{aligned}
$$

In the absence of external fields this satisfies the Landau free energy criterion that it is a minimum at constant $T$ and $V$ when expressed as a function of the order parameters. Thus we identify the Landau free energy as

$$
F=A-\sum_{\mathbf{k}}\left(G_{u} \bar{u}+G_{\Delta} \bar{\Delta}\right)
$$

$F$ must now be expressed in terms of order parameters. By inverting Eqs. (4.28), which relate the order parameters to the fields, we obtain

$$
\begin{array}{r}
F=F_{0}+\sum_{\mathbf{k}} \frac{1}{2}\left[\bar{u}^{\dagger} \cdot M \cdot \bar{u}+\bar{u}^{\dagger} \cdot v \cdot \bar{\Delta}+\bar{\Delta}^{\dagger} \cdot v^{\dagger} \cdot \bar{u}\right. \\
\left.+\bar{\Delta}^{\dagger} \cdot\left(\chi_{0}^{-1}+J+j\right) \cdot \bar{\Delta}\right]+\cdots .
\end{array}
$$

This expression for the Landau free energy as a bilinear function of order parameters depending on the co- efficients in the microscopic Hamiltonian is the goal of this section.

\section{PHASE TRANSITIONS}

There are many examples in which the phase transition from the disordered phase to the orientationally ordered phase is affected by translation-rotation coupling. If the order parameter for the ordered phase has the same symmetry as a phonon displacement coordinate, this coupling will occur at least to some extent, and the transition temperature will be raised. Another result of the involvement of translation-rotation coupling in the phase transition is that the ordered structure differs from the disordered structure by displacements of the centers of mass in addition to having orientational order. The phase transition may then be classified as a mixed displacive and order-disorder transition.

The data in Table I show that it is common for translation-rotation coupling to be involved in phase transitions of ionic lattices and rare in phase transitions of simple molecular crystals. This is because there is a large direct interaction between molecular orientations in molecular crystals, while in ionic compounds the molecular ions are separated by a lattice of counterions, so that the direct interaction $J$ is usually small and the indirect interaction arising from translation-rotation coupling via the counter ions becomes important. A similar situation arises in urea inclusion compounds in which long chain paraffins are confined in a honeycomb lattice made of urea or thiourea (Chatani et al., 1978; Forst et al., 1986). At high temperatures the relative orientations of the paraffin molecules within the honeycomb are random; at lower temperatures they order to a honeycomb structure with a simultaneous distortion of the lattice. Again the direct interaction between adjacent paraffin chains is small, and the ordering is enhanced by an indirect coupling via the distortion of the host lattice (Lynden-Bell, 1993).

From the discussion in Sec. IV.A, we recall that the criterion for the disordered phase to be stable is that the eigenvalues of the $\mathbf{k}$-dependent matrix $\left(1+\chi_{0} \cdot J^{\prime}\right)$ are greater than zero at all $3 N \mathbf{k}$ values. As the temperature is lowered the crystal undergoes a second-order phase transition when one of these eigenvalues becomes equal to zero. The structure of the ordered phase depends on which $\mathbf{k}$ value and which $\Delta_{\mu}$ correspond to this eigenvalue. The single-particle susceptibility matrix $\chi_{0}$ is diagonal and can be written as $\chi_{0}=y_{0} / T$ where $y_{0}$ is a diagonal matrix that depends only weakly on temperature. This means that Curie-Weiss behavior in which

$$
\chi=y_{0} /\left(T-T_{c}\right)
$$

is expected when $J^{\prime}$ has a negative eigenvalue. The transition temperature $T_{c}$ is given by

$$
\begin{aligned}
T_{c} & =\text { Largest }\left\{-y_{0} \cdot J^{\prime}\right\} \\
& =\text { Largest }\left\{y_{0} \cdot\left(v^{\dagger} \cdot M^{-1} \cdot v-J-j\right)\right\},
\end{aligned}
$$


where Largest means the largest eigenvalue of the matrix. The actual phase transition that occurs will be to the phase with symmetry corresponding to the symmetry and $\mathbf{k}$ value of the eigenvalue with the largest $T_{c}$. For example, in $\mathrm{KCN}$ the maximum eigenvalue of any of these matrices is for $T_{2 g}$ symmetry at $\mathbf{k}=0$, while for $\mathrm{NH}_{4} \mathrm{Br}$, the maximum is at a zone boundary value of $\mathbf{k}$, and for sodium nitrite the maximum is an at incommensurate value of $\mathbf{k}$. Both the direct coupling $J$ and the indirect coupling $v^{\dagger} \cdot M^{-1} \cdot v$ are $\mathbf{k}$ dependent. In molecular crystals variations in the direct coupling tend to be the most important, while in ionic lattices the molecules are, to some extent, isolated from each other by the lattice of counterions so the indirect coupling tends to dominate. There are some exceptions to this generalization, for example, $\mathrm{NaO}_{2}$ in which the transition occurs from an orientationally disordered phase to a $\mathrm{Pa} \overline{3}$ ordered phase with no translation-rotation coupling (see the discussion in Sec. V.A), and the urea-paraffin inclusion compounds mentioned above in which translation-rotation coupling is important.

The $\mathbf{k}$ dependence of the indirect coupling near the zone center depends on whether the principal order parameter has an even or odd value of $L$. As $\mathbf{k} \rightarrow 0$, the inverse of the dynamical matrix for acoustic modes, $M^{-1}$, tends to infinity as $k^{-2}$ at low $k$ values. The translationrotation coupling matrix $v$ increases with $k$. We have seen that, for rotator functions with even $L, v$ is linear in $k$ at small $k$, while for rotator functions with odd values of $L, v$ depends quadratically on $k$ in this limit. Thus in the low $\mathbf{k}$ limit, the indirect coupling matrix $v^{\dagger} \cdot M^{-1} v$ in Eq. (5.2) vanishes if $L$ is odd and tends to a constant if $L$ is even. We deduce that rotation-translation coupling cannot facilitate a phase transition at the zone center if the principal order parameter concerned has an odd value of $L$. If $L$ is even, a zone center phase transition will occur if this is the point at which the indirect coupling is a maximum. This depends on the crystal structure and, to some extent, on the details of the intermolecular interactions. The indirect coupling is a maximum at the zone center for coupling to the $L=2$ order parameter in alkali metal cyanides, but is a maximum at the zone boundary for the very different system of urea/alkane inclusion compounds even though the order parameter is also $L=2$ symmetry (Lynden-Bell, 1993). We should emphasize that for even $L$ the limiting value of the indirect coupling $v^{\dagger} \cdot M^{-1} \cdot v$ at $\mathbf{k}=0$ depends on the direction from which the limit is approached. This is a consequence of the fact that the elastic forces are long-ranged, and suggests that there may be a shape dependence of system properties in the low temperature phase for monodomain samples, while in a real crystal there is a breakup of the ordered phase in domains. For additional discussion of this point the reader is referred to Cowley (1976), Folk et al. (1976), and De Raedt et al. (1981). Normally the principal order parameter is a rotator function with the lowest value of $L$ that is totally symmetric in the molecular point group. We have also seen that all the order parameters of centrosymmetric molecules are rotator functions with even $L$, while for other molecular symmetries the lowest allowed value of $L$ is odd. Thus we expect in general that centrosymmetric molecules in ionic lattices will order at the $\Gamma$ point and that noncentrosymmetric molecules will order at some other point in $\mathbf{k}$ space with a resulting enlargement of the unit cell on ordering. In such cases the increase of $v$ and $v^{\dagger}$ with $\mathbf{k}$ outweighs the decrease in $M^{-1}$ and ordering takes place elsewhere, often at a zone boundary point.

In some examples there is clear evidence for competing order parameters. We shall see in the next section that in the alkali cyanides, both $E_{g}$ and $T_{2 g}$ elastic constants behave anomalously and there is a competition between $E_{g}$ and $T_{2 g}$ ordering. Molecular-dynamics calculations of sodium nitrate (Lyndell-Bell et al., 1989) show clear evidence for competition between ordering at the zone boundary $Z$ point (which gives the observed $R \overline{3} c$ structure) and a translation-rotation mediated process at the $F$ point, which would give a monoclinic structure. There is experimental evidence to support monoclinic fluctuations and one can interpret the anomalously small critical exponent in the ordered phase in terms of this competition. For further discussion see Lynden-Bell et al. (1989).

Normand et al. (1990) have shown that materials with multiwell potentials may show a second anomaly or quasitransition (in addition to the order/disorder phase transition) when the temperature is equal to the well depth. This shows up in the specific heat and in spectra. Most orientationally disordered crystals have a barrier between preferred sites that is lower than, or comparable to, the transition temperature for the order/disorder phase transition, but where the crystal field is strong (as in sodium nitrite, for example), one would expect to see such an anomaly. It is a local phenomenon and not affected by translation-rotation coupling.

\section{A. The alkali metal cyanides and superoxides}

In the alkali metal cyanides the disordered cubic phase transforms initially into a phase in which the ions still show head-to-tail disorder. The orientational probability distribution function is sharply peaked in two directions separated by $180^{\circ}$, but is identical in these two directions. Complete order can only occur if order parameters of the lowest value of $L$ are involved. In the case of the cyanides, the order parameter for the first phase transition is a rotator function with $L=2$ rather than $L=1$. There is another transition at lower temperatures to a completely ordered antiferroelectric state (Rowe et al., 1977; Fontaine, 1975). The higher-temperature phase transition involves an even- $L$ rotator function, and as expected from the arguments given above, the intermediate ordered phase occurs at $k=0$. The magnitude of the translation-rotation coupling depends on the details of the intermolecular forces, as follows from Sec. III. In this case we wish to know which is the dominant cou- 
pling, that is, whether the coupling to the $\left(L=2, T_{2 g}\right)$ modes is larger or smaller than the coupling to the ( $L=2, E_{g}$ ) modes. The larger coupling will give the ordered structure. Often a guess can be made by considering the symmetries of the pseudospin variables based on the preferred orientations in the disordered phase. However, the dangers of relying too much on pseudospin states defined by preferred orientations in the disordered state is illustrated by the alkali metal cyanides. In $\mathrm{KCN}$ and $\mathrm{RbCN}$ the preferred orientations in the disordered state are along [111] directions (Rowe et al., 1973; Ehrhardt et al., 1983), giving four pseudospin states with $A_{1 g}$ and $T_{2 g}$ symmetry. There is no pseudospin state with $E_{g}$ symmetry. This is consistent with the observation that order parameters of $T_{2 g}$ are involved in the phase transition. However, in $\mathrm{NaCN}$ the preferred direction of the cyanide ion is along the [100] directions (Fig. 3; see also Fontaine et al., 1977), suggesting that the low-temperature structure should be monoclinic. This is contrary to observation, and computations with model potentials (Mahanti and Sahu, 1982; Sahu and Mahanti, 1982; Michel and Rowe, 1985b) show that translationrotation coupling to $E_{g}$ and $T_{2 g}$ modes depends on a delicate balance of electrostatic and repulsive forces. It is also true that in $\mathrm{NaCN}$ the orientational distribution function shown in Fig. 3, as determined from neutron diffraction data (Rowe et al., 1973), is fairly uniform, with only weak maxima in the [100] directions so that the pseudospin description is even less appropriate here than in many other crystals. On the other hand, the direct calculation of the coupling matrices $v$ from microscopic theory is a difficult task. One has to calculate the singleparticle distribution in a deformed lattice in which collective properties play an important role (Michel and Rowe, 1985b). In addition, our knowledge of intermolecular potentials is incomplete; for example, the electronic charge distribution of the $\mathrm{CN}^{-}$ion in the crystal depends on the instantaneous orientation (Klein and McDonald, 1983).

The principal order parameter of the superoxide ion is a rotator function with $L=2$, so that one would expect from the arguments given above that the ordered phase would occur at $\mathbf{k}=0$. In sodium superoxide the preferred directions are along the [111] axes, giving pseudospin variables of $T_{2 g}$ symmetry. As one would predict from this, the TA phonon dispersion curve in sodium superoxide (Wakabayashi et al., 1982) shown in Fig. 1 is softened near the zone center. However, in spite of this, the phase transition is at the zone boundary and does not involve translation-rotation coupling. Mahanti and Kemeny (1978) have emphasized the importance of the direct electrostatic coupling of the quadrupole moments of the oxygen ions in this phase transition.

\section{B. Ammonium halides and sodium nitrite}

In both these cases the principal order parameter is odd and, as the previous arguments suggest, the ordering transition does not occur at the zone center. Ammonium bromide has a phase transition from the disordered $\beta$ state into the ordered $\gamma$ state that involves the $L=3$ rotator function with $A_{2 u}$ symmetry at $\mathbf{k}=\mathbf{k}_{M}$, where $\mathbf{k}_{M}$ is the $\mathbf{k}$ vector at the $M$ point of the simple cubic lattice. The unit cell in the $\gamma$ phase is therefore twice the size as that of the disordered phase. The involvement of translation-rotation coupling in this transition is manifest by a frozen-in distortion of the ionic lattice (Yamada et al., 1972).

Sodium nitrite provides a particularly interesting example as the phase transition from the disordered state leads to an incommensurate phase. The site symmetry of the nitrite ion is $D_{2 h}$, which allows in-phase coupling of the $L=1$ rotator function describing the dipole orientation to the acoustic mode. In the long-wavelength limit this coupling is proportional to $\mathbf{k}^{2}$ [Eqs. (3.30), (3.40)], so that the indirect orientational coupling matrix $v^{\dagger} M^{-1} v$ is zero at $\mathbf{k}=0$. However, it rises rapidly with $k$ to reach a maximum and then falls as the zone boundary is approached. The formation of an incommensurate phase with a long wavelength $[\mathbf{k} \approx(\pi / 9 a, 0,0)]$ corresponds to the maximum of this function and leads to a freezing-in of coupled displacement and $u$ symmetry orientation variables. The fact that the lowest-order term in this coupling is proportional to $k^{2}$, and that there is no term linear in $k$ involving the relevant orientational variables, is one of the reasons for the existence of an incommensurate phase (Heine et al., 1984).

\section{C. $\mathrm{C}_{60}$ and related systems}

The molecules in the fullerite $\mathrm{C}_{60}$ have the shape of a truncated icosahedron (Kroto et al., 1985) with point group $I_{h}$. At ambient temperatures the crystal structure is fcc (Fleming et al., 1991) with orientational disorder. This disorder was first measured by NMR (Yannoni et al., 1991; Tycko et al., 1991) and by neutron scattering (Neumann et al., 1991). At the unexpectedly high temperature of about $250 \mathrm{~K}$, a first-order phase transition occurs (Dworkin et al., 1991; Heiney et al., 1991) to an orientationally ordered structure with space group $\mathrm{Pa} \overline{3}$ (David et al., 1991; Sachidanandam and Harris, 1991; Harris and Sachidanandam, 1992). The $\mathrm{Pa} \overline{3}$ structure corresponds to a zone boundary ordering at the $X$ point of the disordered fcc structure, rather than the $\Gamma$ point ordering that would arise from bilinear translationrotation coupling. The phase transition is driven by the simultaneous condensation of the three components of a $T_{2 g}$ orientational mode, which is a linear combination of rotator functions belonging to the $L=10$ and $L=6$ manifolds. The mode with $L=10$ is dominant (Michel et al., 1992). The contraction of the lattice at the phase transition (David et al., 1992; Heiney et al., 1992) can be explained by a $V^{R R T}$ term describing the coupling of the square of the zone boundary orientational order parameter to longitudinal acoustic modes at the zone center (Lamoen and Michel, 1993), a particular case of Eq. (3.17). For a review of the structure and dynamics of 
solid $\mathrm{C}_{60}$, we refer the reader to the article by Axe et al. (1994).

Bilinear coupling between orientational modes of $T_{2 g}$ and $E_{g}$ symmetry and acoustic displacements does, of course, occur. This coupling would lead to a ferroelastic phase transition at the zone center as in the cyanides, but the direct repulsive interaction is stronger and the phase transition to the $\mathrm{Pa} \overline{3}$ structure intervenes. The situation is, to a large extent, similar to that encountered in $\mathrm{NaO}_{2}$. There the quadrupole-quadrupole interaction between the $\mathrm{O}_{2}^{-}$ions is responsible for the transition to the $\mathrm{Pa} \overline{3}$ structure (Mahanti and Kemeny, 1979; Zielinski and Parlinski, 1984). However, $C_{60}$ has no quadrupole moment and the relative importance of the direct and indirect interactions depends on the details of the intermolecular potential. Molecular-dynamics studies of this substance have shown that an intermolecular potential that is built up from a sum of atom-atom terms leads to an incorrect tetragonal structure (Cheng and Klein, 1992). In order to stabilize the $\mathrm{Pa} \overline{3}$ structure it is necessary to include interaction centers in the double bonds as well as on the atoms (Sprik et al., 1992a). Preliminary work (Lamoen and Michel, unpublished) suggests that the double bond centers and atomic sites give contributions of opposite sign to the bilinear translation-rotation coupling for rotator functions belonging to the manifold $L=6$, decreasing the importance of this coupling.

In addition to $\mathrm{C}_{60}$, the doped alkali metal compounds $M_{x} \mathrm{C}_{60}$, in which $M$ is an alkali metal ion and $x=0-6$, have attracted much attention (Zhou et al., 1991), especially as some of these compounds are superconducting (Hebard et al., 1991; Stephens et al., 1991). These systems form solid solutions and their stability depends on the nature of the alkali metal, its concentration, and the temperature. See, for instance, Stephens et al. (1992) for $\mathrm{Rb}: \mathrm{C}_{60}$, Weaver et al. (1993) for $\mathrm{K}: \mathrm{C}_{60}$, and Yildirim et al. (1993) for $\mathrm{Na}: \mathrm{C}_{60}$. Of particular interest is the existence or absence of structural phase transitions ( $\mathrm{Zhu}$ et al., 1993). The compounds $M_{1} \mathrm{C}_{60}$ have hightemperature phases with rocksalt structure. In analogy with the alkali-cyanides, we expect ferroelastic phase transitions to occur at lower temperatures. Experimentally the situation is not yet fully explored at the present time. Some compounds, such as $\mathbf{K}_{1} \mathbf{C}_{60}$ (Winter and Kuzmany, 1992), are only stable at high temperatures and decompose to a mixture of $\mathrm{C}_{60}$ and $\mathrm{K}_{3} \mathrm{C}_{60}$ at low temperatures, so that the temperature where a ferroelastic phase transition would occur cannot be reached. In $\mathrm{K}_{3} \mathrm{C}_{60}$, no transition has yet been seen (Christides et al., 1992), although the compound is stable at low temperatures. The absence of a phase transition can be explained by the very strong crystal field, which is due to the cations. This crystal field tends to decrease the singleparticle expectation value $y_{0}(T)$ so that Eq. (5.2) for $T_{c}$ has no solution (Lamoen and Michel, unpublished). Finally, we mention $\mathrm{Rb}_{1} \mathrm{C}_{60}$, in which recently the existence of an orthorhombic phase below $T=350 \mathrm{~K}$ has been reported (Chauvet et al., 1994). We recall that transitions to orthorhombic phases occur in the alkali-cyanides as a consequence of translation-rotation coupling. Given the solid solution character of $M_{x} \mathrm{C}_{60}$, we expect that these systems have analogies with mixed crystals (see Sec. VI.E) and will show orientational glass phases for certain concentrations.

In solid $\mathrm{C}_{70}$, which contains approximately spheroidal molecules, there is evidence for a rhombohedral and a monoclinic phase at temperatures below the fcc disordered phase (Sprik et al., 1992b; Christides et al., 1993; Van Tendeloo et al., 1993; Vaughan et al., 1993). We expect that the phase transition is driven by a coupling of acoustic phonons to the three components of an orientation mode of $T_{2 g}$ symmetry (see Sachidanandam and Harris, 1994). The current status of research is reviewed in a paper by Fischer and Heiney (1993).

\section{SOFTENING OF ELASTIC CONSTANTS}

Elastic constants in orientationally disordered crystals are reduced (or softened) by the indirect coupling of rotator functions $v^{\dagger} \cdot M^{-1} \cdot v$ at the zone center resulting from translation-rotation matrix $v$, coupling rotator functions to long wavelength acoustic displacements. If this coupling results in a phase transition to a more ordered state, then the softening of the corresponding elastic constant is strongly and anomalously temperature dependent, decreasing as the temperature is reduced towards $T_{c}$, but even in orientationally disordered phases where the phase transition does not occur at the zone center, elastic constants may still be significantly softened by translationrotation coupling to rotator functions that are not involved in the phase transition. In this case the temperature dependence is usually normal with small anomalies at the phase transition due to higher-order coupling terms such as $V^{T R R}$ (see Sec. III). In this section we shall discuss the elastic energy of a cubic crystal, taking into account its symmetry. We investigate the effects of bilinear coupling translation-rotation coupling in these crystals. The theory can readily be generalized to crystals of lower symmetry. Higher-order coupling terms could be included in a similar way, but are usually less important causes of softening.

\section{A. Symmetry of elastic constants}

Elastic constants are used to describe the response of a crystal to a small stress applied uniformly over the crystal. They are defined as the ratio of the stress to the strain, where the strain is a measure of the crystal distortion. Elastic constants are softened if the free energy of the crystal is lowered by a change in the orientational probability function when the strain is applied. Such an effect, which is an aspect of translation-rotation coupling, may only occur with orientational variables of the correct symmetry.

In the theory of elasticity (Eshelby, 1956; Landau and Lifschitz, 1986) the strain tensor $\varepsilon$ is defined by 


$$
\mathbf{r}^{\prime}=(1+\varepsilon) \cdot \mathbf{r},
$$

where $\mathbf{r}$ is the position of a point in the crystal before the distortion, and $\mathbf{r}^{\prime}$ is the position of the displaced point. $\varepsilon$ is a symmetric tensor. It is convenient to define six strain components

$$
\begin{aligned}
& \varepsilon_{1}=\varepsilon_{x x}, \quad \varepsilon_{2}=\varepsilon_{y y}, \quad \varepsilon_{3}=\varepsilon_{z z}, \\
& \varepsilon_{4}=\varepsilon_{y z}+\varepsilon_{z y}, \quad \varepsilon_{5}=\varepsilon_{z x}+\varepsilon_{x z}, \quad \varepsilon_{6}=\varepsilon_{x y}+\varepsilon_{y x} .
\end{aligned}
$$

For small distortions the elastic free energy per unit volume, $F_{\text {elas }}$, is given by

$$
F_{\mathrm{elas}}=\frac{1}{2} \sum_{i=1}^{6} \sum_{j=1}^{6} \varepsilon_{i} c_{i j} \varepsilon_{j}
$$

Here we have used the conventional notation $c_{11} \equiv c_{x x, x x}$, $c_{44} \equiv c_{x y, x y}$, etc. for the elastic constants. In principle there are 36 such constants, but the number that are nonzero and independent is restricted by crystal symmetry as $F_{\text {elas }}$ is totally symmetric (Leibfried, 1955). The elastic constants $c_{i j}$ are numbers and so must be equal to zero unless $\varepsilon_{i}$ and $\varepsilon_{j}$ transform as the same irreducible representation of the crystal space group. As we are considering uniform distortions of the crystal, the strains transform as the zone center symmetry species. In cubic groups these are the representations of $O_{h}$, in which any symmetric second-rank tensor has $A_{1 g}, E_{g}$, and $T_{2 g}$ components, giving three independent nonzero elastic constants. The elastic free energy is given by

$$
F_{\text {elas }}=\frac{1}{2}\left[C_{A_{1 g}} \varepsilon_{A_{1 g}}^{2}+C_{E_{g}} \sum_{a=1}^{2} \varepsilon_{E_{g a}}^{2}+C_{T_{2 g}} \sum_{a=1}^{3} \varepsilon_{T_{2 g a}}^{2}\right]
$$

In terms of the conventional labeling

$$
\begin{aligned}
& \varepsilon_{A_{1 g}}=\left(\varepsilon_{1}+\varepsilon_{2}+\varepsilon_{3}\right) / \sqrt{3}, \\
& \varepsilon_{E_{g, a}}=\left(2 \varepsilon_{3}-\varepsilon_{1}-\varepsilon_{2}\right) / 2 \sqrt{3}, \\
& \varepsilon_{E_{g, b}}=\left(\varepsilon_{1}-\varepsilon_{2}\right) / 2, \\
& \varepsilon_{T_{2 g, a}}=\varepsilon_{4}, \\
& \varepsilon_{T_{2 g, b}}=\varepsilon_{5}, \\
& \varepsilon_{T_{2 g, c}}=\varepsilon_{6},
\end{aligned}
$$

and the elastic constants are

$$
\begin{aligned}
& C_{A_{1 g}}=c_{11}+2 c_{12}=c_{x x, x x}+2 c_{x x, y y}=3 B, \\
& C_{E_{g}}=\left(c_{11}-c_{12}\right) / 2=\left(c_{x x, x x}-c_{x x, y y}\right) / 2, \\
& C_{T_{2 g}}=c_{44}=c_{x y, x y},
\end{aligned}
$$

where $B$ is the bulk elastic modulus. In an isotropic medium $C_{E_{g}}=C_{T_{2 g}}$.

One should note that there is a difference between the isothermal elastic constants, which are measured in an experiment in which the stressed crystal is in complete thermal equilibrium with its surroundings, and adiabatic elastic constants, which are measured at constant entropy, as measured in an experiment in which the time scale of the change of stress is short compared with the rate of thermal relaxation. This distinction is present in atomic crystals in which the time scale for thermal relaxation depends on the thermal conductivity of the crystal. In orientationally disordered crystals there is an additional relaxation process, namely the rate of change of molecular orientation. The constants in the free energy expression (6.3) are isothermal in the sense that they correspond to an experiment that is performed slowly enough that the molecular orientational probability distribution changes with the distortion, rather than an experiment that is performed more rapidly than the time scale for molecular reorientation in which the orientational probability distribution remains the same as in andistorted crystal. Thus the observed consequences of translationrotation coupling on both phonons and elastic constants depends on the time scale of the change of distortion compared with the time scale of molecular reorientation. No softening of elastic constant is observed in a dynamic experiment in which the molecules reorient on a slower time scale than the vibrational period. Values of elastic constants are often measured from phonon dispersion curves or Brillouin scattering and are often found to show softening due to translation-rotation coupling, although such measurements are usually termed adiabatic. The time scale for molecular reorientation is usually short compared to that for full thermal relaxation, so that one must consider the regime in which only orientational order reaches thermal equilibrium. We shall return to this question of the time scale of orientational relaxation in the next section on phonon dynamics.

\section{B. Softening of elastic constants}

Softening of the isothermal elastic constants $C_{A_{1 g}}$, $C_{T_{2 g}}$, and $C_{E_{g}}$ of a cubic crystal occurs if there is a significant change in the amount of the orientational order of the corresponding symmetry when the crystal is distorted. There are always orientational variables of the correct symmetry as all rotator functions with even $L$ values have some components of $E_{g}$ and $T_{2 g}$ symmetries, and those with $L=4,6, \ldots$ have $A_{1 g}$ components in addition. Thus $\left(c_{11}-c_{12}\right)$ and $c_{44}$ are softened by changes in the contribution of rotator functions of the corresponding symmetry in the orientational distribution function as the crystal is distorted. Similarly, the bulk modulus is reduced by changes in the $A_{1 g}$ components of $L=4,6$, etc. variables. The points of interest are how large the softening is, which elastic constant is softened most, and what is the temperature dependence of the softening. These questions may be addressed by considering the changes in the orientational distribution functions as consequences of translation-rotation coupling to long wavelength phonons. 
One can see that there is a close connection between the elastic constants and the acoustic phonons by considering the distortion of a crystal caused by an acoustic phonon displacement with a very long wavelength. If such an acoustic phonon has $\mathbf{k}$ in direction $\alpha$ and atomic displacements in direction $\beta$, the local strain in the crystal has an $\alpha \beta$ component $\varepsilon_{\alpha \beta}$. To calculate the isothermal elastic constants from microscopic considerations we shall use the expression for the Landau free energy $F(\bar{u}(\mathbf{k}), \bar{\Delta}(\mathbf{k}))$ derived in Sec. IV [Eq. (4.2)]. We first rewrite this in terms of the lattice-displacement order parameters $\bar{u}(\mathbf{k})$, alone, and then equate the longwavelength limit of the resulting equation with Eq. (6.2) for the free energy of the crystal under a uniform elastic deformation.

To obtain an expression for the Landau free energy in terms of the phonon displacement variables, we minimize $F(\bar{u}, \bar{\Delta})$

$$
\begin{gathered}
F(\bar{u}, \bar{\Delta})=F_{0}+\sum_{\mathbf{k}} \frac{1}{2}\left[\bar{u}^{\dagger} \cdot M \cdot \bar{u}+\bar{u}^{\dagger} \cdot v \cdot \bar{\Delta}+\bar{\Delta}^{\dagger} \cdot v^{\dagger} \cdot \bar{u}\right. \\
\left.+\bar{\Delta}^{\dagger} \cdot\left(\chi_{0}^{-1}+J+j\right) \cdot \bar{\Delta}\right]
\end{gathered}
$$

with respect to the orientational order parameters $\bar{\Delta}$ at constant displacements $\bar{u}$ in an analogous way to the derivation of its dependence on the orientational order parameters [Eq. (4.36)]. This gives

$$
\begin{aligned}
F & =F_{0}+\sum_{\mathbf{k}} \frac{1}{2}\left[\bar{u}^{\dagger} \cdot M \cdot \bar{u}-\bar{u}^{\dagger} \cdot v \cdot\left(\chi_{0}^{-1}+J+j\right)^{-1} \cdot v^{\dagger} \cdot \bar{u}\right] \\
& =F_{0}+\sum_{\mathbf{k}} \frac{1}{2} \bar{u}(\mathbf{k})^{\dagger} \cdot D(\mathbf{k}) \cdot \bar{u}(\mathbf{k})
\end{aligned}
$$

where the matrix $D(\mathbf{k})$ is known as the dressed dynamical matrix and is equal to

$$
D(\mathbf{k})=M(\mathbf{k})-v(\mathbf{k}) \cdot\left[\chi_{0}^{-1}+J(\mathbf{k})+j\right]^{-1} \cdot v^{\dagger}(\mathbf{k})
$$

This shows that the effect of the translation-rotation coupling matrix $v$ is to soften the dressed dynamical matrix $D$ relative to the bare dynamical matrix $M$. If the coupling is sufficiently strong, an eigenvalue of $D$ becomes equal to zero, in which case there is no restoring force and the crystal becomes unstable with respect to a distortion along the corresponding eigenfunction $\bar{u}$. It can be shown after some algebraic manipulation that $D \equiv \chi_{u u}^{-1}$ [Eq. (4.31)].

To obtain the elastic constants we now compare the long-wavelength limit of this with Eq. (6.2), to obtain

$$
L t_{k \rightarrow 0} D_{i j}^{a c}(\mathbf{k})=\rho^{-1} \sum_{p, q} c_{i p, j q} k_{p} k_{q},
$$

where $\rho$ is the mass density. The reader is referred to Ashcroft and Mermin (1976, Chap. 22) for more details of this derivation. Next we have to solve Eq. (6.9) where $D(\mathbf{k})$ is given by Eq. (6.8). Due to the summation over indices $p$ and $q$, this solution is not trivial. It can be achieved by choosing $\mathbf{k}$ in particular directions. For instance, for the case of cubic crystals [compare with Eq. (6.12) below], we have for $\mathbf{k}=\left(k_{x}, 0,0\right)$, the diagonal elements $\rho D_{x x}(\mathbf{k})=c_{x x, x x} k_{x}^{2}, \rho D_{y y}(\mathbf{k})=c_{y x, y x} k_{x}^{2}$, etc. We also remind the reader of the discussion of the $\mathbf{k} \rightarrow 0$ limit in the introductory part of Sec. V. In expression (6.8), the bare dynamical matrix for the acoustic modes is quadratic in $k$ near $\mathbf{k}=0$. The matrix $\left[\chi_{0}^{-1}+J(\mathbf{k})+j\right]^{-1}$ is diagonal at $\mathbf{k}=0$ in the space of rotator functions and has eigenvalues $\left[\chi_{\mu}^{-1}+J_{\mu}(0)+j_{\mu}\right]^{-1}$, which depend on the symmetry and $L$ value of the rotator function $\Delta_{\mu}$ involved. As $\mathbf{k}$ tends to zero, $v$ is linear in $k$ for coupling to rotator functions with even- $L$ values and quadratic for odd- $L$ values. The softening is quadratic in $v$ and so will be either quadratic or quartic in $\mathbf{k}$. We are looking for terms that are quadratic in $\mathbf{k}$, so it follows that only translation-rotation coupling to rotator functions with even $L$ can soften the elastic constants. We have already argued on general symmetry grounds that as strains can only couple to rotator functions of the same symmetry species, the elastic constant $C_{\Gamma}$ is softened by translation-rotation coupling to rotator functions of symmetry species $\Gamma$ alone.

We shall now demonstrate this for a particular example, the alkali metal cyanides. A microscopic calculation of the elastic constants on the basis of these equations was first carried out for the orientationally disordered phase of KCN (Michel and Naudts, 1977a, 1977b; Mahanti and Sahu, 1982). Since this example is a model case for many of the concepts we are developing in the present paper, we shall now derive the results.

We start by taking the long-wavelength limit of Eqs. (3.26) and (3.27) for the coupling matrices for an alkali metal cyanide. To first order in $\mathbf{k}$ one obtains for the $E_{g}$ coupling matrix to the acoustic modes (using Eq. 3.14)

$$
v(\mathbf{k})^{\dagger}=-\frac{2 i A a}{m^{1 / 2}}\left[\begin{array}{ccc}
k_{x} & k_{y} & -2 k_{z} \\
\sqrt{3} k_{x} & -\sqrt{3} k_{y} & 0
\end{array}\right],
$$

and for the $T_{2 g}$ coupling matrix

$$
v(\mathbf{k})^{\dagger}=-\frac{2 i B a}{m^{1 / 2}}\left[\begin{array}{ccc}
0 & k_{z} & k_{y} \\
k_{z} & 0 & k_{x} \\
k_{y} & k_{x} & 0
\end{array}\right]
$$

If one includes only the nearest-neighbor interactions, the integrals $A$ and $B$ are the same as those in Eqs. (3.26) and (3.27); but even if longer-range interactions are added, the matrices have the same form with modified values of $A$ and $B . a$ is half the length of the side of the cubic unit cell. In the case of a cubic crystal, the longwavelength limit of the bare dynamical matrix, $M(k)$, for coupling to acoustic modes is 


$$
M(\mathbf{k})=\rho^{-1}\left[\begin{array}{ccc}
k_{x}^{2} c_{11}^{0}+\left(k_{y}^{2}+k_{z}^{2}\right) c_{44}^{0} & k_{x} k_{y}\left(c_{12}^{0}+c_{44}^{0}\right) & k_{x} k_{z}\left(c_{12}^{0}+c_{44}^{0}\right) \\
k_{y} k_{x}\left(c_{12}^{0}+c_{44}^{0}\right) & k_{y}^{2} c_{11}^{0}+\left(k_{z}^{2}+k_{x}^{2}\right) c_{44}^{0} & k_{y} k_{z}\left(c_{12}^{0}+c_{44}^{0}\right) \\
k_{z} k_{x}\left(c_{12}^{0}+c_{44}^{0}\right) & k_{z} k_{y}\left(c_{12}^{0}+c_{44}^{0}\right) & k_{z}^{2} c_{11}^{0}+\left(k_{x}^{2}+k_{y}^{2}\right) c_{44}^{0}
\end{array}\right]
$$

where $\rho=m /\left(2 a^{3}\right)$ is the mass density. The dressed dynamical matrix $D$ has the same form but with the dressed elastic constants $c$ replacing the bare constants $c^{0}$. We now calculate the contribution to the softening of $M$ due to coupling to the $L=2, E_{g}$ rotator functions. For these functions the matrix $\left[\chi_{0}^{-1}+J(\mathbf{k})+j\right]$ is a constant times the unit matrix, and $v^{\dagger}$ and $v$ are given by Eq. (6.10) and its conjugate transpose. Substituting this into Eq. (6.9) for the dressed dynamical matrix, we obtain

$$
D-M=-\frac{4 A^{2} a^{2}}{m\left[\chi_{E_{g}}^{-1}+J_{E_{g}}(0)+j_{E_{g}}\right]}\left[\begin{array}{ccc}
4 k_{x}^{2} & -2 k_{x} k_{y} & -2 k_{x} k_{z} \\
-2 k_{x} k_{y} & 4 k_{y}^{2} & -2 k_{y} k_{z} \\
-2 k_{z} k_{x} & -2 k_{z} k_{y} & 4 k_{z}^{2}
\end{array}\right] .
$$

To determine the elastic constants, we use Eq. (6.9), and after taking the $\mathbf{k} \rightarrow 0$ limit and using $\rho=m /\left(2 a^{3}\right)$, we find

$$
\begin{aligned}
& c_{11}=c_{11}^{0}-\frac{8 A^{2}}{a\left[\chi_{E_{g}}^{-1}+J_{E_{g}}(0)+j_{E_{g}}\right]}, \\
& c_{12}=c_{12}^{0}+\frac{4 A^{2}}{a\left[\chi_{E_{g}}^{-1}+J_{E_{g}}(0)+j_{E_{g}}\right]},
\end{aligned}
$$

and $c_{44}$ is unchanged by this term. A similar calculation for the $T_{2 g}$ variables yields no further change in $c_{11}$ and $c_{12}$, and

$$
c_{44}=c_{44}^{0}-\frac{2 B^{2}}{a\left[\chi_{T_{2 g}^{-1}}^{-1}+J_{T_{2 g}}(0)-j_{T_{2 g}}\right]} .
$$

$c_{44}$ is the $T_{2 g}$ elastic constant, which is, as expected, softened by coupling to the $T_{2 g}$ rotator variable. The $E_{g}$ elastic constant is softened,

$$
\left(c_{11}-c_{12}\right) / 2=\left(c_{11}^{0}-c_{12}^{0}\right) / 2-\frac{6 A^{2}}{a\left[\chi_{E_{g}}^{-1}+J_{E_{g}}(0)+j_{E_{g}}\right]},
$$

while the $A_{1 g}$ combination $c_{11}+2 c_{12}$ is unchanged.

Equation (6.8) shows that the effects of coupling to other rotator functions can just be added. In particular, coupling to $L=4$ variables, which includes a rotator function of $A_{1 g}$ symmetry, does give a small softening of the bulk compressibility as well as additional softening of $C_{E_{g}}$ and $C_{T_{2 g}}$ due to $L=4$ rotator functions of $E_{g}$ and $T_{2 g}$ symmetry, respectively.

In general, we have for an elastic constant for strains of symmetry species $\Gamma$

$$
C_{\Gamma}=C_{\Gamma}^{0}\left(1-\sum_{L} \frac{A_{L \Gamma}^{2}}{\left[\chi_{L, \Gamma}^{-1}+J_{L, \Gamma}(0)+j_{L, \Gamma}\right]}\right),
$$

where the sum is over all rotator functions of that symmetry species, and $A_{L \Gamma}$ is a coupling coefficient. However, there is usually one dominant contribution. Where this is true, the elastic constant is reduced to zero when the corresponding orientational susceptibility diverges, that is, when $\left(1+J_{\Gamma}^{\prime} \chi_{\Gamma}^{0}\right)$ is equal to zero (see Sec. IV.A).

\section{Temperature dependence}

The dominant temperature dependence of the elastic constants arises from the single particle susceptibilities introduced in Sec. II.D, which can be written $\chi_{\Gamma}=y_{\Gamma} / T$, where $y_{\Gamma}$ is only weakly temperature dependent (Michel and Naudts, 1977a, 1977b; Mahanti and Sahu, 1982). If there is one dominant contribution to the softening, the equation above may be rewritten as

$$
C=C^{0} \frac{\left(T-T_{c}\right)}{\left(T-T_{0}\right)},
$$

with the transition temperature $T_{c}$ obtained from solution of the equation

$$
T_{c}=-\left(J+j-A^{2} / C_{\Gamma}^{0}\right) y_{\Gamma}\left(T_{c}\right),
$$

and the temperature $T_{0}$ determined by

$$
T_{0}=-(J+j) y_{\Gamma}\left(T_{c}\right) \text {. }
$$

For the interpretation of experimental data on the temperature behavior of elastic constants near phase transitions, one sometimes uses a formula of the form of Eq. (6.19) (Rehwald, 1973; Rehwald et al., 1977; Ginzburg et al., 1980), interpreting $T_{c}$ as the phase transition temperature and $T_{0}$, known as the clamped crystal transition temperature, as the temperature at which the orientational order would occur in the absence of translationrotation coupling. Although this has the same form as the general expression derived above, there is no restriction in the derivation that either $T_{c}$ or $T_{0}$ need be positive. If $T_{c}$ is positive, then we agree that it should be interpreted as a real transition temperature or, if negative, as a virtual transition temperature. The usual interpretation of $T_{0}$, however, is not quite correct in our opinion. The conventional interpretation of $T_{0}$ ignores the role of the self-interaction $j$, which is a consequence of the translation-rotation coupling. The self-term is always 
positive, while the strength and sign of $J$ depends on the structure of the crystal. For instance, in the cubic phase of $\mathrm{KCN}, J_{E_{g}}$ is negative at $\mathbf{k}=0$, while $J_{T_{2 g}}$ is positive but smaller than $j$ (Michel and Rowe, 1985b). Since $y$ is always positive, one finds in both cases that $T_{0}<0$ in agreement with experiment (Rehwald, 1973; Rehwald et al., 1977; Ginzburg et al., 1980).

\section{Some examples}

In this section some examples of the effect of translation-rotation coupling on elastic constants are discussed in order of the $L$ value concerned.

In all the alkali metal cyanides, the cyanide ions (symmetry $C_{\infty v}$ ) behave in the high-temperature disordered phase as if they were centrosymmetric (symmetry $D_{\infty h}$ with a principal order parameter of $L=2$ ). The site symmetry is octahedral and the cubic disordered phase has three independent elastic constants: the bulk compressibility $B\left(A_{1 g}\right.$ symmetry), the shear constant $c_{44}$ with $T_{2 g}$ symmetry, and the uniaxial stress constant $C_{E_{g}}=\left(c_{11}-c_{12}\right) / 2$. Both the latter are smaller than for the corresponding alkali halides and decrease as the temperature is lowered as shown in Fig. 7. The bulk
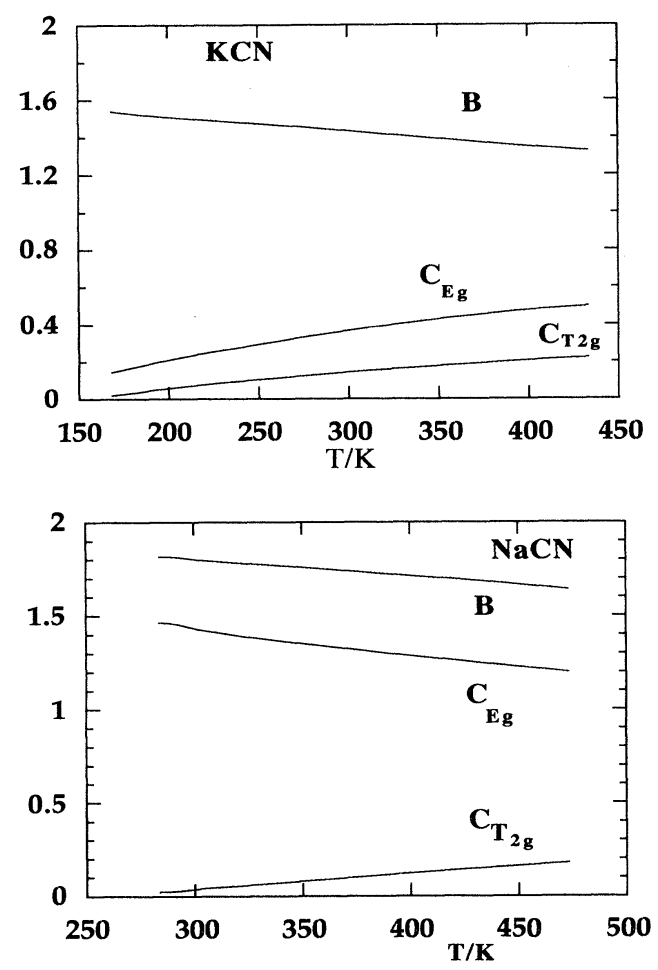

FIG. 7. Temperature dependence of the elastic constants of $\mathrm{NaCN}$ and $\mathrm{KCN}$ in their orientationally disordered phases. $B$ is the bulk compressibility and $C_{E_{g}}, C_{T_{2 g}}$ are the elastic constants for uniaxial and shear distortions, respectively (see text). Values are in units of $10^{10} \mathrm{Jm}^{-3}$. The phase transitions are at $280 \mathrm{~K}$ and $168 \mathrm{~K}$, respectively. Data from Haussühl et al. (1977). compressibility behaves normally, decreasing slightly as the temperature is raised. This shows the coupling of the $L=2$ rotator functions to $E_{g}$ and $T_{2 g}$ strains, and the absence of significant coupling (which could only arise from higher- $L$ values) to $A_{1 g}$ strains. $c_{44}$ tends to zero at the phase transition temperature, while $C_{E_{g}}$ tends to zero at a lower temperature. This is because $y_{E_{g}} J_{E_{g}}^{\prime}<y_{T_{2 g}} J_{T_{2 g}}^{\prime}$ so that $T_{c}=y_{T_{2 g}} J_{T_{2 g}}^{\prime}$. The elastic behavior in the hightemperature phase is similar for Rb (Ehrhardt, Press, and Lefebvre, 1983), K, and Na cyanides (Rowe et al., 1975). The transition temperature, however, decreases with increasing size of cation, the disordered phase in $\mathrm{RbCN}$ being most stable because the $\mathrm{CN}^{-}$ion has the most room for reorientation in this compound. In the theory that has been outlined before, the influence of the cations enters not only in the coefficients of the bilinear coupling matrix, but also in the expressions for the crystal field, which determines the parameter $y_{0}$ in the expressions for the orientational susceptibility [Eqs. (4.29) and (2.54)] and transition temperature [Eq. (5.2)].

The role of cations is even more pronounced in the alkali metal nitrites $\mathrm{MNO}_{2}$. We have already seen (Sec. III.H) that in $\mathrm{NaNO}_{2}$, the $\mathrm{NO}_{2}^{-}$behaves as a pseudospin with symmetry $B_{1 u}$, and that translation-rotation coupling does not lead to softening of the elastic constants. On the other hand, in the compounds with larger cations (Richter and Pistorius, 1972; Hirotsu et al., 1981; Hohlwein et al., 1986), the nitrite ion has much more orientational freedom, and so has additional orientational variables of even $L$ and the correct symmetry to soften the elastic constants. For example, one can imagine that rapid rotation of the $\mathrm{NO}_{2}^{-}$around an axis parallel to the O-O vector leads to an effective $D_{\infty h}$ symmetry, with $L=2$ rotator variables. The coupling to acoustic lattice modes would then be similar to the one already encountered in the alkali metal cyanides. Softening of the elastic constant $c_{44}$ with $T_{2 g}$ symmetry has been observed in $\mathrm{RbNO}_{2}$ by Hirotsu et al. (1981).

In the materials containing tetrahedral molecules, ammonium halides, $\mathrm{CH}_{4}$, and the carbon tetrahalides, the rotator functions with lowest- $L$ values have $L=3$, which are the wrong parity to soften the elastic constants, although they do form the order parameters for the phase transition. Nevertheless, softening of the elastic constants by rotation-translation coupling at the $\Gamma$ point may occur as a result of coupling to $L=4$ rotator functions. One anticipates that this should be comparatively small due to the high value of $L$ involved. It should also show no connection with the phase transition as it involves a completely different set of rotator functions. Earlier we have shown how the symmetry of pseudospin states may be used to provide an indication of which symmetry is most involved in translation-rotation coupling. As already stated in Sec. II.E there are two pseudospin variables in ammonium chloride and ammonium bromide that have $A_{1 g}$ and $A_{2 u}$ symmetry. This suggests that any bilinear coupling effect on the elastic con- 


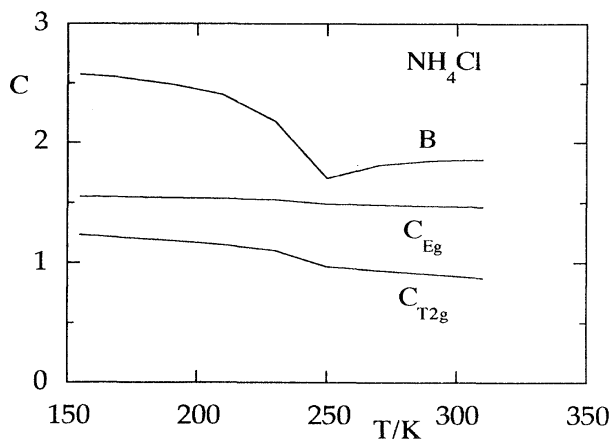

FIG. 8. Temperature dependence of the elastic constants of $\mathrm{NH}_{4} \mathrm{Cl}$ above and below the order/disorder phase transition that occurs at $240 \mathrm{~K} . B$ is the bulk compressibility and $C_{E_{g}}, C_{T_{2 g}}$ are the elastic constants for uniaxial and shear distortions, respectively (see text). Values are in units of $10^{10} \mathrm{Jm}^{-3}$. Data from Garland and Renard (1966c).

stants will be very small as there are no pseudospin variables of the required symmetry. The anomalous behavior of the elastic constants of ammonium chloride near $T_{c}$ shown in Fig. 8 can be well explained (Garland and Renard, $1966 \mathrm{a}, 1966 \mathrm{~b})$ by a theory in which the coupling is quadratic in the orientational order parameters and linear in the acoustic phonons. When we go to higher order in the order parameter coupling, the conditions governing the $\mathbf{k}$ values of the order parameters involved are less restrictive. For example, pairs of zone-boundary order parameters can couple to functions at the $\Gamma$ point. However, it is by no means always necessary to invoke such higher-order coupling, and it was found that the compressible Ising model used for ammonium chloride is not sufficient to explain the temperature dependence of the elastic constants in $\mathrm{NH}_{4} \mathrm{Br}$ (Garland and Yarnell, 1966). There is a small change at $T_{c}$ due to trilinear terms, but, in addition, $C_{E_{g}}$ already shows a marked decrease at $15^{\circ}$ above $T_{c} \approx 230 \mathrm{~K}$. This suggests the possibility of a contribution from the bilinear coupling to $L=4$ rotator functions with $E_{g}$ symmetry. So far the orientational distribution function has only been analyzed (Seymour and Pryor, 1970) for diffraction data taken at $296 \mathrm{~K}$. In order to check our conjecture, one would need diffraction data close to $T_{c}$.

In the plastic phase of $\mathrm{CD}_{4}$ the phase transition concerns ordering of $L=3$ variables and occurs away from the zone center (odd $L$ ), while the zone-center softening of the elastic constants is due to the lowest even- $L$ orientational variable, that is, $L=4$. The coupling of $L=4$ rotator functions to acoustic displacements has been used (Wonneberger and Hüller, 1987) to explain the reduction of the elastic constants. The theoretical results are in satisfactory agreement with experiment (Marx and Simmons, 1984). Similar arguments have been used to explain the elastic constants in the carbon tetrahalides. In the rotator-function description, the lowest- $L$ rotator functions have $L=3$, and it is primarily these that are involved in the phase transition that occurs at a zoneboundary point. However, in the discrete variable description of these crystals (see Sec. II.E) pseudospin variables of $E_{g}$ symmetry are involved in addition to the $T_{2 u}$ functions, suggesting that when the $L=4$ rotator functions are considered, $C_{E_{g}}$ will be softened more than $C_{T_{2 g}}$. This has indeed been observed for $\mathrm{CD}_{4}, \mathrm{CBr}_{4}$, and $\mathrm{CCl}_{4}$ (Zuk et al., 1989, 1990). Although these constants are softened compared with the values for argon, in which there is no possibility of translation-rotation coupling, their temperature dependence is normal and they are not involved with the phase transition.

Adamantane provides another example in which the effective symmetry is higher than the true molecular symmetry. The molecule has symmetry $T_{d}$ so that the primary orientational order parameters would be expected to have $L=3$. However, examination of a molecular model shows that the symmetry is nearly octahedral, so that $L=4$ variables are probably the most important. The disordered phase has fcc symmetry and the tetragonal ordered phase has a unit cell that is derived from the fcc structure by an $E_{g}$ distortion in the (100) direction. It is not a zone-center transition, however, as the unit cell contains two adamantane molecules (Donohue and Goodman, 1965; Nordman and Schmitkons, 1967). The importance of translation-rotation coupling to the $L=4$, $E_{g}$ variables is shown in the temperature dependence of the elastic constants (Damien, 1975), which is shown in Fig. 9. In the disordered phase the bulk compressibility and $c_{44}$ both behave normally, i.e., they increase as the temperature is lowered towards the phase transition, but $\left(c_{11}-c_{12}\right) / 2$ behaves anomalously, decreasing as the temperature is lowered. However, the apparent limit of stability $T_{c}$ is considerably lower than the phase transition temperature. We may interpret this as showing that there is a strong zone-center coupling to the $L=4, E_{g}$ variables, which would lead to a zone-center ordering at

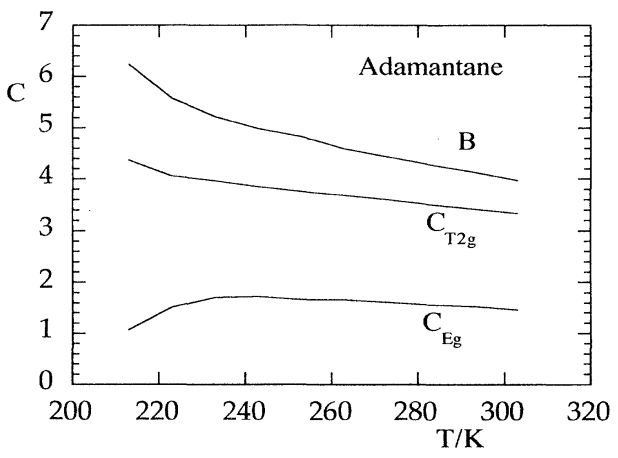

FIG. 9. Temperature dependence of the elastic constants of adamantane in the disordered phase. $B$ is the bulk compressibility and $C_{E_{g}}, C_{T_{2 g}}$ are the elastic constants for uniaxial and shear distortions, respectively (see text). Values are in units of $10^{9} \mathrm{Jm}^{-3}$. The order/disorder phase transition is at $208.6 \mathrm{~K}$. Data from Damien (1975). 
a temperature about $30-50 \mathrm{~K}$ lower than the observed zone-boundary phase transition if the latter could be avoided.

Recent experiments on $\mathrm{KPF}_{6}$ (Knorr, 1992, private communication) have shown that in this crystal a drastic softening of the elastic constant $c_{44}$ occurs near the transition from the disordered cubic phase to a low symmetry structure. The octahedral symmetry of the $\mathrm{PF}_{6}^{-}$ions implies that the lowest-order rotator functions belong to the $L=4$ manifold. Since these include a $T_{2 g}$ representation in the cubic site group, the softening of $c_{44}$ is in full agreement with our theoretical considerations. Sulphur hexafluoride $\left(\mathrm{SF}_{6}\right)$ possesses an orientationally disordered phase above $96 \mathrm{~K}$ with a body-centered-cubic lattice structure. The molecular symmetry is again octahedral, but since there are no counterions, the coupling of the $L=4$ orientational modes to acoustic lattice displacements, although symmetry allowed, is expected to be weak. Measurements of the elastic constants have only been published for temperatures above $187 \mathrm{~K}$ (Kiefte et al., 1988). It would be desirable to extend these measurements to lower temperatures to check whether there is a softening of $c_{44}$.

So far there are only a few experiments on the elastic properties of solid $\mathbf{C}_{60}$. Measurements of Young's Modulus on single crystals of $\mathrm{C}_{60}$ (Shi et al., 1992) reveal an upward jump in the sound velocity at $T \approx 260 \mathrm{~K}$ with decreasing temperature and a sharp increase in the corresponding sound attenuation. More recent experiments (Schranz et al., 1993a, 1993b), performed at much lower frequencies $(0.1 \mathrm{~Hz}-50 \mathrm{~Hz})$, measure an effective elastic constant $C(\omega)=C^{\prime}(\omega)+i C^{\prime \prime}(\omega)$ for sound waves propagating in the [111] direction. These experiments can be described qualitatively (Lamoen, 1993) on the basis of the $V^{R R T}$ coupling mentioned before. More complete information exists about the acoustic phonons that have been measured in inelastic neutron scattering from a single crystal of $\mathrm{C}_{60}$ (Pintschovius et al., 1992). As already mentioned in Sec. V.C, the direct repulsive interaction between $\mathrm{C}_{60}$ molecules is dominant (Michel et al., 1992). On the other hand, since we have seen that the existence of a rhombohedral phase in $\mathrm{C}_{70}$ and an orthorhombic phase in $\mathbf{R b C}_{60}$ is a consequence of translation-rotation coupling with condensation of the $T_{2 g}$ variables at the zone center, we expect softening of the elastic constant $C_{T_{2 g}}=c_{44}$ in these latter compounds.

In deriving expression (6.10) for the softening of elastic constants, we have restricted ourselves to a cubic crystal. However, the same approach may be extended to crystals of other symmetries. For example, Sahu (1986) has calculated the softening of the elastic constants in $\beta-\mathrm{NaN}_{3}$, an orientationally disordered crystal with a rhombohedral structure. A good fit to the experimental data (Kushida and Terhune, 1984) can be obtained with a suitable intermolecular potential. Another example of a ferroelastic crystal is aniline hydrobromide $\left(\mathrm{C}_{6} \mathrm{H}_{5} \mathrm{NH}_{3} \mathrm{Br}\right)$. Here the elastic constant $c_{55}$ is found to soften in the high-temperature orthorhombic phase as the temperature is lowered towards the transition point for the phase change to the low temperature monoclinic structure (Sawada et al., 1980).

\section{E. Mixed crystals}

So far we have considered the softening of elastic constants due to translation-rotation coupling in ideal periodic crystals. In mixed crystals with substitutional disorder it is possible to vary the importance of translation-rotation coupling by changing the substitutional disorder. The most prominent examples of this category of mixed crystals are the alkali metal halidescyanides $M(\mathrm{CN})_{x} X_{1-x}$ and the mixed alkali metal cyanides $\left(M_{1}\right)_{x}\left(M_{2}\right)_{1-x} \mathrm{CN}$. Here $X$ is a halogen and $M$ are alkali metal ions. Depending on the nature of the substitutional ions and on the concentration, these systems exhibit very rich phase diagrams (Lüty, 1981; Elschner et al., 1985; Knorr and Loidl, 1985; Rowe et al., 1986). A theoretical explanation in terms of translation-rotation coupling has been given by Michel and Theuns (1989). At a sufficiently large degree of substitutional disorder, the ferroelastic phase transition that appears in the ideal compounds $M C N$ is suppressed (Lüty, 1981). The temperature evolution of the elastic constant $c_{44}$ exhibits a characteristic minimum (Satija and Wang, 1978). A similar behavior (softening and subsequent hardening as a function of decreasing $T$ ) is found in the transverse acoustic phonon frequencies of $\mathrm{K}(\mathrm{CN})_{x} X_{1-x}$ near $x=0.5$ as measured by inelastic neutron scattering (Rowe et al., 1979), and in addition the neutron scattering law reveals a central peak. These phenomena can be interpreted as being caused by the transition from a normal orientationally disordered crystal at high temperatures to an orientational glass at low temperatures. Substitutional disorder generates static random strain fields that strongly influence the molecular orientations (Michel, 1986; Fossum and Garland, 1988). In the glassy phase the time scale for molecular reorientation is very slow, so that only static effects of translation-rotation coupling are important. As we have seen earlier in this section, the softening of elastic constants is only manifest if the time scale for molecular orientation is fast compared with that of the measuring technique; hence we see softening followed by hardening as the temperature is lowered. We shall show in the next section that a similar argument applies to the phonon spectra. In the glass phase, the characteristic shape of the elastic line in $\mathbf{Q}$ space, as shown by equal intensity contours, is a direct consequence of bilinear translationrotation coupling (Michel and Rowe, 1980). These concepts have also been found to be relevant for the understanding of diffuse $x$-ray scattering in new organic conductors based on TMDTDSF (tetramethyldithiadiselenafulvalene) molecules (Liu et al., 1993). Orientational glasses are today the subject of intense research ac- 
tivity. For recent reviews, we refer the reader to Höchli et al. (1990) and Binder and Reger (1992).

We expect that many concepts that have been introduced and used for the characterization and description of orientational glasses will be useful for our understanding of mixed crystals of $\mathrm{C}_{60}$ and $\mathrm{C}_{70}$ and of fullerides $M_{x} \mathrm{C}_{60}$ and $\left(M_{1}\right)_{x}\left(M_{2}\right)_{y} \mathrm{C}_{60}$ in which $M_{1}$ and $M_{2}$ are two different alkali metals. There are experimental indications of orientational glass properties in solid $\mathrm{C}_{60}$ below $T \approx 90 \mathrm{~K}$ (David et al., 1992; Gugenberger et al., 1992). Undoubtedly this will be a very active field of research in the coming years.

\section{DYNAMIC EFFECTS AND PHONONS}

Since the elastic constants and the acoustic phonons are determined by dynamic measurements, and the phonons can be investigated in neutron scattering experiments, it is important to investigate the frequency dependence of the effects of translation-rotation coupling. In general the response of a many body system to a timedependent perturbation can be formulated in terms of time- and space-dependent correlation functions. The knowledge of the latter functions is equivalent to the knowledge of dynamic equations for the relevant physical quantities (Kadanoff and Martin, 1963). We first derive the dynamical equations for the system of coupled displacements and orientational variables. There are two methods of deriving these equations: a direct one that starts from the microscopic Hamiltonian and uses Green's-function methods (Michel and Naudts, 1978), and a second, more macroscopic one that starts with the free energy (Yamada et al., 1974b; Courtens, 1976). We will use here the latter method, which goes back to Onsager (1931a, 1931b) and Machlup and Onsager (1953).

We write the Lagrangian density $\mathcal{L}$ for the coupled system in the form

$$
\mathcal{L}=\mathcal{T}-F,
$$

where $\mathcal{T}$ is the kinetic energy of the displacements

$$
\mathcal{T}=\frac{1}{2} \sum_{\mathbf{k}} \dot{u}^{\dagger}(\mathbf{k}) \dot{u}(\mathbf{k}),
$$

and $F=F[\bar{\Delta}, \bar{u}]$ is the Landau free energy of the crystal [see Eq. (4.36)]. In this section we allow the order parameters, that is, the mean values of the rotator functions $\bar{\Delta}$ and the phonon displacements $\bar{u}$, to be time dependent. In the remainder of this section we shall simplify the notation by writing $u$ for $\bar{u}$. We recall that $\dot{u}$ stands for the time derivative $\partial u / \partial t$. In Eq. (7.1) we only consider the contribution of $\dot{u}$ to the kinetic energy as the orientational contribution is usually overdamped (Courtens, 1976). The equations of motion are

$$
\frac{\partial}{\partial t}\left[\frac{\partial \mathcal{L}}{\partial \dot{a}_{i}}\right]-\frac{\partial \mathcal{L}}{\partial a_{i}}=\frac{\partial f}{\partial \dot{a}_{i}},
$$

where the set of variables $\left\{a_{i}\right\}$ include both the $u(\mathbf{k})$ and the $\Delta(\mathbf{k})$, and $f$ is a dissipative potential describing the relaxation of the variables. The most important contribution to relaxation arises from the orientational variables and is written

$$
f=\frac{1}{2} \Gamma \sum_{\mathbf{k}} \dot{\Delta}^{\dagger}(\mathbf{k}) \dot{\Delta}(\mathbf{k}),
$$

where $\Gamma$ is an orientational relaxation coefficient. Using the expression for the free energy from Eq. (4.36), Eq. (7.3) gives

$$
\begin{aligned}
& \ddot{u}+M \cdot u+v \cdot \Delta=0, \\
& v^{\dagger} \cdot u+X \cdot \Delta=-\Gamma \dot{\Delta},
\end{aligned}
$$

where $X$ is a matrix

$$
X=\left[\chi_{0}^{-1}+J+j\right] .
$$

These equations describe a system of oscillators $u$, which are coupled to orientational variables with the same $\mathbf{k}$ vector. If there were no coupling between the displacement and orientational variables $(v=0)$, then Eq. (7.5) shows that the displacements would oscillate harmonically with frequencies given by the square roots of the eigenvalues of the dynamical matrix $M$, while the rotator variables would not oscillate, but would decay exponentially with rates given by the eigenvalues of the matrix $\lambda$ defined by

$$
\dot{\Delta}=-\Gamma^{-1} \cdot X \cdot \Delta=-\lambda \cdot \Delta .
$$

In order to solve Eqs. (7.5) and (7.6) and to describe the relaxation behavior in a convenient way (Kadanoff and Martin, 1963), we introduce the Laplace transforms of the variables,

$$
a_{i}(\mathbf{k}, z)=-i \int_{0}^{\infty} e^{i z t} a_{i}(\mathbf{k}, t) d t
$$

where $z=\omega+i \epsilon$ with $\omega$ being the frequency and $\epsilon \rightarrow 0^{+}$. With the boundary conditions $a_{i}=a_{i, 0} \neq 0$, and $\dot{a}_{i}=0$ at $t=0$, we obtain for each value of $\mathbf{k}$

$$
\begin{aligned}
& \left(z^{2}-M\right) u(z)-v \cdot \Delta(z)=z u_{0}, \\
& (z+i \lambda) \Delta(z)+i v^{\dagger} \lambda X^{-1} u(z)=\Delta_{0} .
\end{aligned}
$$

Here the first equation describes the oscillatory behavior of the displacement field density $u$ (the translational phonons), while the second equation describes the relaxation of the orientational density. These motions are coupled by the translation-rotation coupling matrix $v$. Eliminating the rotator variables, we obtain an equation for the displacements

$$
\left[z^{2}-D(\mathbf{k}, z)\right] u(\mathbf{k}, z)=I(\mathbf{k})
$$

where

$$
D(\mathbf{k}, z)=M(\mathbf{k})-i \frac{v \cdot \lambda \cdot X^{-1} \cdot v^{\dagger}}{z+i \lambda}
$$

and

$$
I(\mathbf{k})=u_{0}(\mathbf{k})+v(\mathbf{k}) \Delta_{0}(\mathbf{k}) /(z+i \lambda) .
$$


These solutions show the effects of translation-rotation coupling on the phonon spectrum, which is described by the displacement response function $u(\mathbf{k}, z)$. Peaks in the spectrum occur when the real part of $\omega^{2}-D(\mathbf{k}, \omega)$ is zero. One can distinguish two limiting regimes of behavior (Yamada et al., 1974b). In the fast-relaxation regime when $\lambda$, the inverse of the relaxation time of the rotator variables is much larger than $\omega$, the frequency of the observation, Eq. (7.13), leads to the replacement $D(\mathbf{k}, z) \rightarrow D(\mathbf{k})$, where $D(\mathbf{k})$ is the dressed dynamical matrix given in Eq. (6.8). As we discussed in Sec. VI, the effect of the translation-rotation coupling is to reduce the values of the eigenvalues of this matrix, and hence to soften the elastic constants. In the present context we deduce that in the fast relaxation limit, the resonances correspond to the eigenvalues of the dressed dynamical matrix, so that the modes appear to be softened. Figure 10(b) shows the form of the resulting spectrum. In the other limit of slow relaxation where $\lambda<\omega$, $D(\mathbf{k}, z) \rightarrow M(\mathbf{k})$ and the phonon resonances are not shifted by the translation-rotation coupling. In this limit the scattering law exhibits a narrow central "relaxational" peak [see Fig. 10(a)] in addition to the unshifted Brillouin doublet. This is due to orientational relaxation and has its origin in the $z=-i \lambda$ pole of the second term on the right hand side of Eq. (7.13). A given material may show both regimes in different regions of $\mathbf{k}$ space as the bare

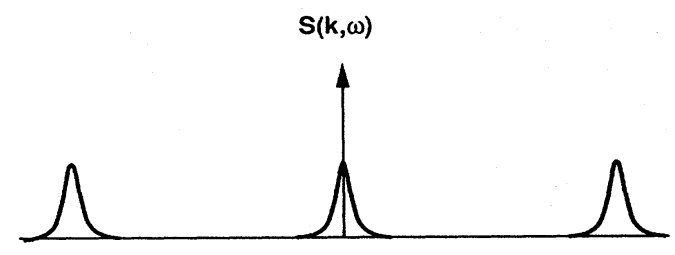

(a)

$\omega$

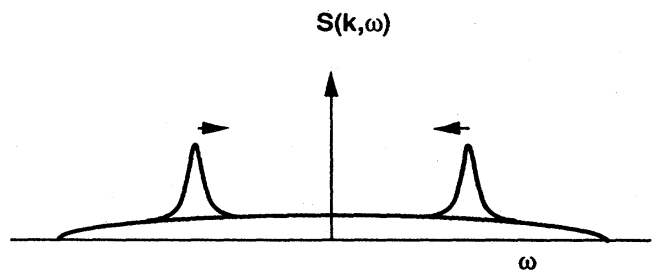

(b)

FIG. 10. Form of the phonon spectrum in the presence of translation-rotation coupling: (a) the slow relaxation limit where the Brillouin lines are unshifted, and a central relaxation peak appears; (b) the fast relaxation limit where the phonon resonances are softened and the relaxation peak is too broad to be seen. resonance frequencies change relative to $\lambda$. For a more complete discussion of the frequency dependence of the scattering law, the reader is referred to the previously quoted papers. An extensive discussion of the frequency dependence of ultrasonics and Brillouin scattering is given in a recent review on structurally incommensurate crystal phases (Cummins, 1990).

Inelastic neutron scattering (Yamada et al., 1974a) shows that $\mathrm{NH}_{4} \mathrm{Br}$ exhibits the triple peak structure characteristic of the slow relaxation regime. The narrow central band is due to the slowly reorienting ammonium ions that are coupled to the zone-boundary $\mathrm{TA}_{2}$ phonon modes. These give the unsoftened outer pair of peaks. Notice that the general theoretical considerations apply to all parts of $\mathbf{k}$ space; in this case the zone-boundary phonon mode is coupled to an orientational mode of the same symmetry, which can be described as an antiferrorotational mode. $\mathrm{NaNO}_{2}$ is another example of an order-disorder phase transition, in which the dynamics are characterized by the presence of critical slowing down of orientational motion (Hatta et al., 1966; Sakurai et al., 1970) and the absence of phonon softening (Durand et al., 1982). For a derivation of orientational and translational motion in the paraelectric and incommensurate phases, we refer the reader to Fivez and Michel (1983).

The fast relaxation regime has been seen in the alkali cyanides, in which the inelastic neutron-scattering spectrum exhibits a soft phonon doublet (Rowe et al., 1975; Ehrhardt et al., 1983). Here the orientational relaxation is fast enough that no central peak is found in the inelastic spectrum, and all the information on the orientational dynamics is hidden in the details of the widths and softening of the phonon peaks. It is possible to pass from the fast relaxation regimes to the (almost) slow relaxation limit in $\mathrm{KCN}$ by increasing the momentum transfer $\mathbf{Q}$ in the neutron scattering (Rowe et al., 1978). Another experimental procedure for changing from the fast to the slow relaxation regime has recently been used in the case of ammonium deuterium oxalate hemihydrate $\left(\mathrm{ND}_{4} \mathrm{DC}_{2} \mathrm{O}_{4}, 1 / 2 \mathrm{D}_{2} \mathrm{O}\right)$. There the application of a pressure of 5 kbar brings about a change in the relaxation regime and consequently in the neutron scattering spectrum (Krauzman et al., 1992).

From the discussion in this section it is clear that the measured phonon frequencies depend on the frequency regime of the method of measurement. Phonon branches that appear hard in neutron scattering may be soft when measured by Brillouin scattering in which the condition $\lambda>\omega$ is more easily realized.

\section{DIELECTRIC BEHAVIOR OF CRYSTALS WITH DEFORMABLE IONS}

The anomalous dielectric behavior found in the orientationally disordered $\alpha$ phase of ammonium iodide and in solid solutions of ammonium and potassium iodide provides an interesting illustration of the way in which col- 
lective rotator coordinates can be related to macroscopic properties. This phase has the sodium chloride structure with ammonium ions on sites with $\mathrm{O}_{h}$ symmetry. Neutron scattering experiments on $\mathrm{K}_{1-x}\left(\mathrm{NH}_{4}\right)_{x} \mathrm{I}$ at low ammonium ion concentration reveal that there is a large orientation-dependent interaction between the $\mathrm{NH}_{4}^{+}$ions (Bostoen et al., 1989). Since symmetry arguments indicated that elastic interactions are negligible, these authors suggested that the crystal field potential might induce a dipole in the ammonium ion. Subsequently, Fehst et al. (1990) measured the dielectric constants of these materials as a function of temperature, composition, and frequency. At room temperature the dielectric constant $\kappa$ of the pure compound is equal to $11 \pm 1$, which is somewhat larger than that of sodium chloride $(\kappa=6.1)$ or potassium iodide $(\kappa=5.1)$. Over the accessible temperature range it increases as the temperature is lowered according to a Curie-Weiss law. At a lower temperature the substance undergoes a first-order phase transition to the $\beta$ phase similar to the $\beta$ phase of ammonium bromide discussed earlier.

The dielectric constant of a material is a measure of the dipole susceptibility at $\mathbf{k}=0$. We have seen in Sec. IV that Curie-Weiss behavior,

$$
\chi=\frac{C_{\mathrm{CW}}}{T-T_{c}},
$$

where $C_{\mathrm{CW}}$ and $T_{c}$ are constants, is typical behavior of a crystal with coupled rotator functions [Eq. (5.1)]. The critical temperature $T_{c}$ is given by Eq. (5.2). If we use these equations for coupling to the molecular dipole moment (a rotator function with $L=1$ ), $T_{c}$ and the CurieWeiss constant $C_{\mathrm{CW}}$ are given by

$$
\begin{aligned}
& T_{c}=-J^{\prime}\left\langle\mu^{2}\right\rangle_{0}, \\
& C_{\mathrm{CW}}=y_{0}=\left\langle\mu^{2}\right\rangle_{0} / k_{B} .
\end{aligned}
$$

Fehst et al. (1990) fitted their data with this expression and obtained a value of the effective molecular dipole moment $\mu$ of $1.4 D$ and a negative value for $T_{c}$, which implies a positive effective coupling constant $J^{\prime}$.

It is surprising that there is a molecular contribution of this kind, as an ammonium ion has no intrinsic dipole moment. The observed dipole moment is thought to arise from distortions of the molecular framework, and an induced electronic dipole caused by the crystal field. An alternative possibility is that it is the result of displacements of the ions from the center of their sites. We think that this latter possibility is unlikely, although not impossible, and we know of no examples in which random off-center displacements of ions are found in orientationally disordered crystals.

If the molecule is in a " $T_{d}$ " orientation (Fig. 2) where its planes of symmetry coincide with those of the site, no dipole can be induced, but if it rotates about one bond by $60^{\circ}$ into one of the " $C_{3 v}$ " orientations, a dipole is induced in the [111] direction in which the unique bond points. In this orientation three bonds point near iodide atoms and one in a [111] direction. As the molecule rotates between different " $C_{3 v}$ " orientations, the direction of the induced dipole rotates by a larger angle than the molecule moves.

We write the induced dipole moment $\mu$ of a molecule (both electronic and due to distortions of the nuclear framework), which is due to the perturbation of the molecule by the crystal field $V^{R}$ as

$$
\mu=\alpha(\omega) V^{R},
$$

where $\alpha$ is a generalized polarizability that may be expanded in terms of site-symmetry-adapted functions with different $L$ values. The instantaneous value of $\alpha$ depends on the orientation of the molecule relative to the site axes that may be expressed in terms of the Euler angles $\omega$. We may write the polarizability in crystal axes in terms of the polarizability in molecular axes using rotator functions

$$
\alpha(\omega)=\sum_{\mu} \Delta_{\mu}(\omega) \alpha^{L}
$$

where the molecular polarizabilities $\alpha^{L}$ are constants for a particular molecule that transform according to the totally symmetric representation of the molecular group. The label $\mu=L, \tau, A_{1}$ on the rotator function is a composite label as used earlier [Eq. (2.21)], which shows the symmetry species in both the site and molecular point groups ( $\tau, A_{1}$, and the $L$ value). We note that the $L$ values of the polarizabilities concerned are restricted by the rules for coupling angular momenta (Edmonds, 1960; Brink and Satchler, 1968). As the resultant dipole moment has $L=1$, the Clebsch-Gordan series shows that a term in the crystal field with $L=L_{c}$ may only interact with polarizabilities with $L=L_{c}+1, L_{c}$, and $L_{c}-1$. The lowest possible $L$ value for the ammonium ion is $L=3$, so that polarizabilities exist for this $L$ value and higher ones. The crystal-field potential in the $\mathrm{O}_{h}$ site group has terms with $L=4,6, \ldots$, each of which transforms as the totally symmetric representation of the site group. When the $L=3$ polarizability is coupled with the $L=4$ terms in $V^{R}$, the resulting dipole moment does indeed transform as $L=1$. In a dielectric experiment, the total dipole moment of the sample is observed. This is the $\mathbf{k}=0$ function

$$
\widetilde{\mu}=\widetilde{\Delta}_{\mu}(\mathbf{k}=0) \alpha^{(3)} V^{(4)}
$$

The dielectric constant is determined by the mean-square fluctuation in the quantity

$$
\left\langle\tilde{\mu}^{2}(\mathbf{k}=0)\right\rangle=\left(V^{(4)} \alpha^{(3)}\right)^{2}\left\langle\left[\widetilde{\Delta}_{L=3, T_{1 u}, A_{1}}(\mathbf{k}=0)\right]^{2}\right\rangle .
$$

Thus the constants in the Curie-Weiss temperature dependence of the dielectric constant [Eqs. (8.1) and (8.2)] are proportional to the susceptibility of these $L=3$ zone-center rotator functions. In the earlier part of this article we saw that translation-rotation coupling does not affect orientational coupling of rotator functions with odd values of $L$ at the zone center, so the value of $J^{\prime}$ in 
Eq. (8.2) is solely due to direct coupling of the $L=3$ rotator functions as discussed in Sec. II, and is unaffected by translation-rotation coupling.

The frequency dependence of the real and imaginary parts of the dielectric constant is determined by the Fourier transform of the time correlation function of the total dipole moment $\tilde{\mu}$. In orientationally disordered crystals this will be affected by molecular reorientation, and although the dipole moment is a $L=1$ property, it is the effect of molecular reorientation on the rotator functions in Eq. (8.4) that matters. Thus in the dielectric experiment, one is monitoring the decay of the $L=3$ Legendre function $\left\langle P_{3}(\cos \theta)\right\rangle$ rather than the more usual $\langle\cos \theta\rangle$.

A similar effect should occur in the $\beta$ phase with $\mathrm{CsCl}$ structure, although the mean-square effective dipole moment is likely to be considerably lower as the preferred orientations in this phase are " $T_{d}$ " in which there is no induced dipole moment. Experimentally the dielectric constant does seem to increase as the temperature is lowered, but less rapidly than in the $\alpha$ phase. The magnitude of the dielectric constant, however, does not drop as much as one might expect from this argument.

\section{CONCLUDING REMARKS}

The aim of this article has been to show how translation-rotation coupling in orientationally disordered crystals can influence phase transitions, affect the value of elastic constants, and change the appearance of phonon spectra. We have shown how these effects can be calculated quantitatively from a knowledge of intermolecular potentials, and we have demonstrated how the use of symmetry arguments can lead to qualitative explanations. Symmetry, however, only tells one whether a coupling is allowed, not how large it is. For example, the symmetry of the order parameter associated with the phase transition from the disordered to the ordered phase depends on the $\mathbf{k}$ value and the symmetry of the orientational order parameters corresponding to the maximum value of the total coupling, direct and indirect (mediated by translation-rotation coupling). Symmetry tells us whether the latter is zero at particular points such as the zone center and the zone boundary, but not where it is a maximum. More detailed calculations are needed to predict which order parameter condenses at the phase transition. Once this is known (from experiment or calculation), one can use symmetry arguments to predict which, if any, elastic constant tends to vanish near the transition temperature.

We have shown how it is possible to establish a link between macroscopic thermodynamic quantities (elastic constants, free energies) and microscopic intermolecular potentials. The latter are not always well known, especially in ionic compounds. In the next few years it is likely that $a b$ initio calculations that take into account the electronic structure of the molecule in the crystal and the way in which it changes with molecular displacement will improve our knowledge of intermolecular potentials. The concepts and methods we have used are not restricted to potentials that are the sum of atom-atom terms but can readily be generalized to other forms of potential. In particular the expressions in terms of symmetry-adapted rotator functions remain valid.

Although we have not discussed the trilinear coupling terms between displacements and orientation variables in detail, the treatment in Sec. III can readily be extended to such terms. In particular we have mentioned some examples in which the $V^{T R R}$ terms between principal orientation order parameters at zone-boundary points can couple with elastic strains, giving anomalous expansion (e.g., in $\mathrm{C}_{60}$, sodium nitrate and calcium carbonate) and changes in the lattice constants and elastic parameters at the order/disorder phase transition.

We hope that our treatment has shown some universal aspects-due to molecular symmetry-of translationrotation coupling. We have illustrated these features with both linear and nonlinear molecules. As materials of increasing complexity are synthesized [such as the fullerites and related compounds, anisotropic organic conductors (Moret et al., 1982), etc.] in which translation rotation is important, it is expected that the concepts that have been developed in the present article will find further useful applications in the near future. In particular we believe that these concepts are essential to the understanding of the role of orientational degrees of freedom in such materials.

\section{REFERENCES}

Allen, M. P., and D. J. Tildesley, 1987, Computer Simulation of Liquids (Clarendon, Oxford).

Ashcroft, N. W., and N. D. Mermin, 1976, Solid State Physics (Holt, Reinhart and Winston, New York).

Atoji, M., 1958, Acta Crystallogr. 11, 827.

Axe, J. D., S. C. Moss, and D. A. Neumann, 1994, in Solid State Physics, edited by H. Ehrenreich and F. Spaepen (Academic, New York), Vol. 48, p. 150.

Bergman, D. J., and B. I. Halperin, 1976, Phys. Rev. B 13, 2145. Bethe, H., 1929, Ann. Phys. (Germany) 3, 133.

Binder, K., and J. D. Reger, 1992, Adv. Phys. 41, 547.

Bischofsberger, T., and E. Courtens, 1975, Phys. Rev. 35, 1451.

Boissier, M., R. Vacher, D. Fontaine, and R. M. Pick, 1978, J. Phys. (France) 39, 161.

Born, M., and K. Huang, 1956, Dynamical Theory of Crystal Lattices (Clarendon, Oxford).

Bösch, M., and W. Känzig, 1975, Helv. Phys. Acta 48, 743.

Bostoen, C., G. Coddens, and W. Wegener, 1989, J. Chem. Phys. 91, 6337.

Bouckaert, L. P., R. Smoluchowski, and E. Wigner, 1936, Phys. Rev. 50, 58.

Bounds, D. G., M. L. Klein, and I. R. McDonald, 1981, Phys. Rev. Lett. 46, 1682.

Boyle, L., 1990, private communication.

Bradley, C. J., and A. P. Cracknell, 1972, The Mathematical

Theory of Symmetry in Solids (Clarendon, Oxford).

Breymann, W., and R. M. Pick, 1988, Europhys. Lett. 6, 227. 
Breymann, W., and R. M. Pick, 1989, Europhys. Lett. 8, 429.

Briels, W. J., A. P. J. Jansen, and A. van der Avoird, 1986, Adv. Quantum Chem. 18, 131.

Brink, D. M., and G. R. Satchler, 1968, Angular Momentum (Clarendon, Oxford).

Carpenter, M. A., 1988, in Physical Properties and Thermodynamic Behavior of Minerals, edited by E. Salje (Reidel, Dordrecht), p. 265.

Chatani, Y., H. Anraku, and Y. Taki, 1978, Mol. Cryst. Liq. Cryst. 48, 219.

Chauvet, O., G. Oszlànyi, L. Forro, R. W. Stephens, M. Teyze, G. Faigel, and A. Jànossy, 1994, Phys. Rev. Lett. 72, 2721.

Cheng, A., and M. L. Klein, 1992, Phys. Rev. B 45, 1889.

Christides, C., D. A. Neumann, K. Prassides, J. R. D. Copley, J. J. Rush, M. J. Rosseinsky, D. W. Murphy, and R. C. Haddon, 1992, Phys. Rev. B 46, 12088.

Christides, C., I. M. Thomas, T. J. S. Dennis, and K. Prassides, 1993, Europhys. Lett. 22, 611.

Coulon, G., and M. Descamps, 1980, J. Phys. C 13, 2947.

Courtens, E., 1976, J. Phys. Lett. 37, L21.

Cowley, R. A., 1976, Phys. Rev. B 13, 4877.

Cummins, H. Z., 1990, Phys. Rep. 185, 211.

Damien, J. C., 1975, Solid State Commun. 16, 1271.

David, W. I. F., R. W. Ibberson, T. J. S. Dennis, J. P. Hare, and K. Prassides, 1992, Europhys. Lett. 18, 219.

David, W. I. F., R. M. Ibberson, J. C. Matthewman, K. Prassides, T. J. S. Dennis, J. P. Hare, H. W. Kroto, R. Taylor, and D. R. M. Walton, 1991, Nature 35, 147.

De Raedt, B., K. Binder, and K. H. Michel, 1981, J. Chem. Phys. 75, 2977.

Devonshire, A. F., 1936, Proc. R. Soc. London A 153, 601.

Dolling, G., B. M. Powell, and V. F. Sears, 1979, Mol. Phys. 37, 1859.

Donohue, J., and S. H. Goodman, 1967, Acta Crystallogr. 22, 352.

Dove, M. T., and R. M. Lynden-Bell, 1986, J. Phys. C 19, 3343.

Dultz, W., 1974, Solid State Commun. 15, 595.

Durand, D., F. Denoyer, M. Lambert, L. Bernard, and R. Currat, 1982, J. Phys. (France) 43, 149.

Dworkin, A., H. Szwarc, S. Leach, J. P. Hare, T. J. S. Dennis, H. W. Kroto, R. Taylor, and D. R. M. Walton, 1991, C. R. Acad. Sci. Sér. II 312, 979.

Edmonds, A. R., 1960, Angular Momentum in Quantum Mechanics (Princeton University, Princeton).

Ehrhardt, K. D., and K. H. Michel, 1981(a), Phys. Rev. Lett. 46, 1197.

Ehrhardt, K. D., and K. H. Michel, 1981b, Z. Phys. B 41, 329.

Ehrhardt, K. D., W. Press, and G. Heger, 1983, Acta Crystallogr. B 39, 171.

Ehrhardt, K. D., W. Press, and J. Lefebvre, 1983, J. Chem. Phys. 78, 1476.

Elliott, R. J., R. T. Horley, W. Hayes, and S. R. P. Smith, 1972, Proc. R. Soc. Lond. A 328, 217.

Elschner, S., K. Knorr, and A. Loidl, 1985, Z. Phys. B 61, 209.

Eshelby, D., 1956, Solid State Physics, Vol. III (Academic, New York), p. 79.

Feder, J., and E. Pytte, 1973, Phys. Rev. B 8, 3978.

Fehst, I., R. Böhmer, W. Ott, A. Loidl, S. Haussühl, and C. Bostoen, 1990, Phys. Rev. Lett. 64, 3139.

Ferrario, M., M. L. Klein, R. M. Lynden-Bell, and I. R. McDonald, 1987, J. Chem. Soc. Faraday Trans. II 83, 2097.

Fivez, J., and K. H. Michel, 1983, Z. Phys. B 51, 127.

Fischer, J. E., and P. A. Heiney, 1993, J. Phys. Chem. Solids (in press).
Fleming, R. M., T. Siegrist, P. M. Marsh, B. Hessen, A. R. Kortan, D. W. Murphy, R. C. Haddon, R. Tycko, G. Dabbagh, A. M. Mujsce, M. L. Kaplan, and S. M. Zahurak, 1991, Mater. Res. Soc. Symp. Proc. 206, 691.

Folk, R., H. Iro, and F. Schwabl, 1976, Z. Phys. B 25, 69.

Fontaine, D., 1975, C. R. Acad. Sci. Ser. B 281, 443.

Fontaine, D., R. Pick, and M. Yvinec, 1977, Solid State Commun. 21, 1095.

Fontaine, D., and R. M. Pick, 1979, J. Phys. (France) 40, 1105.

Forst, R., H. Boysen, F. Frey, and H. Jagodzinski, 1986, J. Phys. Chem. Solids 47, 1098.

Frenkel, D., and B. M. Mulder, 1985, Mol. Phys. 55, 1171.

Fossum, J. O., and C. W. Garland, 1988, Phys. Rev. Lett. 60, 592.

Garland, C. W., and R. Renard, 1966a, J. Chem. Phys. 44, 1120. Garland, C. W., and R. Renard, 1966b, J. Chem. Phys. 44, 1125. Garland, C. W., and R. Renard, 1966c, J. Chem. Phys. 44, 1130. Garland, C. W., and C. F. Yarnell, 1966, J. Chem. Phys. 44, 1112.

Gehring, G. A., and K. A. Gehring, 1975, Rep. Prog. Phys. 38, 1.

Ginzburg, V. L., A. P. Levanyuk, and A. A. Sobyanin, 1980, Phys. Rep. 57, 151.

Gugenberger, F., R. Heid, C. Meingart, P. Adelmann, M. Braun, H. Wühl, M. Haluska, and H. Kuzmany, 1992, Phys. Rev. Lett. 69, 3774.

Harris, A. B., and R. Sachidanandam, 1992, Phys. Rev. B 46, 4944.

Hatta, I., T. Sakudo, and S. Sawada, 1966, J. Phys. Soc. Jpn. 21, 1612.

Haussühl, S., 1973, Solid State Commun. 13, 147.

Haussühl, S., J. Eckstein, K. Recker, and F. Wallrafen, 1977, Acta Crystallogr. A 33, 847.

Hebard, A. F., M. J. Rosseinsky, R. C. Haddon, D. W. Murphy, S. H. Glarum, T. T. M. Palstra, A. P. Ramirez, and A. R. Kortan, 1991, Nature 350, 660.

Heine, V., R. M. Lynden-Bell, J. D. C. McConnell, and I. R. M. McDonald, 1984, Z. Phys. B 56, 1199.

Heine, V., and J. D. C. McConnell, 1981, Phys. Rev. Lett. 46, 1092.

Heiney, P. A., J. E. Fischer, A. R. McGhie, W. J. Romanov, A. M. Denenstein, J. P. McCauley, A. B. Smith, and D. E. Cox, 1991, Phys. Rev. Lett. 66, 2911.

Heiney, P. A., G. B. M. Vaughan, J. E. Fischer, N. Coustel, D. E. Cox, J. R. D. Copley, D. A. Neumann, W. A. Kamitakahara, K. M. Creegan, D. M. Cox, J. P. McCauley, and A. B. Smith, 1992, Phys. Rev. B 45, 4544.

Herzberg, G., 1945, Infra-Red and Raman Spectra of Polyatomic Molecules (Van Nostrand, New York), p. 122.

Hirotsu, S., M. Miyamoto, and I. Yamamoto, 1981, Jpn. J. Appl. Phys. 2, Lett. 20, 917.

Höchli, U. T., K. Knorr, and A. Loidl, 1990, Adv. Phys. 39, 405.

Hohlwein, D., A. Hoser, and W. Prandl, 1986, Z. Kristallogr. 177, 93.

Horner, H., 1974, in Dynamical Properties of Solids, edited by G. K. Horton and A. A. Maradudin (North-Holland, Amsterdam), p. 474.

Hüller, A., and J. W. Kane, 1974, J. Chem. Phys. 61, 3599.

Hüller, A., and J. C. Raich, 1982, J. Chem. Phys. 77, 2038. International Crystallographic Tables, 1992, Vol. C, edited by A. J. C. Wilson (Kluwer, Academic, Dordrecht), p. 503. James, H. M., and T. A. Keenan, 1959, J. Chem. Phys. 31, 12. Kadanoff, L. P., and P. C. Martin, 1963, Ann. Phys. (N.Y.) 24, 
419.

Kanamori, J., 1960, J. Appl. Phys. 31, 145.

Kataoka, Y., K. Okada, and T. Yamamoto, 1973, Chem. Phys. Lett. 19, 365.

Kiefte, H., R. Penney, and M. J. Clouter, 1988, J. Chem. Phys. 88, 5846.

Klein, M. L., and I. R. McDonald, 1983, J. Chem. Phys. 79, 2333.

Klein, M. L., I. R. McDonald, and Y. Ozaki, 1982, Phys. Rev. Lett. 48, 1197.

Klein, M. L., I. R. McDonald, and Y. Ozaki, 1983, J. Chem. Phys. 79, 5579.

Knorr, K., 1992, private communication.

Knorr, K., and A. Loidl, 1985, Phys. Rev. B 31, 5387.

Kobashi, K., R. D. Etters, and T. Yamamoto, 1984, J. Phys. C $17,13$.

Krasser, W., U. Buchenau, and S. Haussüh1, 1976, Solid State Commun. 18, 287.

Krauzman, M., M. Debeau, R. M. Pick, M. Quilichini, P. Launois, and F. Moussa, 1992, J. Phys. I (France) 2, 329.

Krätschmer, W., L. D. Lamb, K. Fostiropoulos, and D. R. Huffman, 1990, Nature 347, 354.

Kroto, H. W., J. R. Heath, S. C. O'Brien, R. F. Curl, and R. E. Smalley, 1985, Nature 318, 162.

Kurki-Suonio, K., 1967, Ann. Acad. Sci. Fenn. A VI, 263.

Kushida, T., and R. W. Terhune, 1984, Phys. Rev. B 30, 3554.

Lamoen, D., 1993, Ph.D. thesis (University of Antwerp).

Lamoen, D., and K. H. Michel, 1993, Phys. Rev. B 48, 807.

Landau, V. L., 1937, Phys. Z. Sowjetunion 11, 26 (Part I); 11, 545 (Part II).

Landau, L. D., and E. M. Lifschitz, 1980, Statistical Physics (Pergamon, New York).

Landau, L. D., and E. M. Lifschitz, 1986, Theory of Elasticity, 3rd Edition (Pergamon, New York).

Leibfried, G., 1955, in Handbuch der Physik-Encyclopedia of Physics, Vol. 7, Part I, edited by S. Flügge (Springer-Verlag, Heidelberg), p. 104.

Liu, Q., S. Ravy, J. P. Pouget, I. Johanssen, and K. Bechgaard, 1993, J. Phys. I (France) 3, 803 (Part I); 3, 821 (Part II).

Loidl, A., K. Knorr, J. Daubert, W. Dultz, and W. J. Fitzgerald, 1980, Z. Phys. 38, 153.

Longuet-Higgins, H. C., 1962, Mol. Phys. 6, 445.

Lüty, F., 1981, in Defects in Insulating Crystals, edited by V. M. Turkevivich and K. K. Shvarts (Springer-Verlag, Heidelberg), p. 69.

Lynden-Bell, R. M., 1993, Mol. Phys. 79, 313.

Lynden-Bell, R. M., M. Ferrario, I. R. McDonald, and E. Salje, 1989, J. Phys. Condens. Matter 1, 6523.

Lynden-Bell, R. M., M. L. Klein, and I. R. McDonald, 1984, Z. Phys. B 54, 325.

Lynden-Bell, R. M., I. R. McDonald, and M. L. Klein, 1983, Mol. Phys. 48, 1093.

Lynden-Bell, R. M., and A. J. Stone, 1989, Mol. Simul. 3, 271.

Machlup, S., and L. Onsager, 1953, Phys. Rev. 91, 1512.

Mahanti, S. D., and G. Kemeny, 1979, Phys. Rev. B 20, 2105.

Mahanti, S. D., and D. Sahu, 1982, Phys. Rev. Lett. 48, 936.

Marx, S. V., and R. O. Simmons, 1984, J. Chem. Phys. 81, 944.

Michel, K. H., 1981, Phys. Rev. B 24, 3998.

Michel, K. H., 1986, Phys. Rev. Lett. 57, 2188.

Michel, K. H., J. R. D. Copley, and D. A. Neumann, 1992, Phys. Rev. Lett. 68, 2929.

Michel, K. H., and E. Courtens, 1981, Phys. Rev. B 23, 513.

Michel, K. H., and H. De Raedt, 1976, J. Chem. Phys. 65, 977.

Michel, K. H., and J. Naudts, 1977a, Phys. Rev. Lett. 39, 212.
Michel, K. H., and J. Naudts, 1977b, J. Chem. Phys. 67, 547.

Michel, K. H., and J. Naudts, 1978, J. Chem. Phys. 68, 216.

Michel, K. H., and K. Parlinski, 1985, Phys. Rev. B 31, 1823.

Michel, K. H., and J. M. Rowe, 1980, Phys. Rev. B 22, 1417.

Michel, K. H., and J. M. Rowe, 1985a, Phys. Rev. B 32, 5818.

Michel, K. H., and J. M. Rowe, 1985b, Phys. Rev. B 32, 5827.

Michel, K. H., and T. Theuns, 1989, Phys. Rev. B 40, 5761.

More, M., and R. Fouret, 1980, Faraday Discuss. 69, 75.

More, M., J. Lefebvre, and B. Hennion, 1984, J. Phys. (France) 45, 303.

More, M., J. Lefebvre, B. Hennion, B. M. Powell, and C. M. E. Zeyen, 1980, J. Phys. C 13, 2833.

Moret, R., J. P. Pouget, R. Comès, and K. Bechgaard, 1982, Phys. Rev. Lett. 49, 1008.

Neumann, D. A., J. R. D. Copley, R. L. Cappelletti, W. A. Kamitakahara, R. M. Lindstrom, K. M. Creegan, D. M. Cox, W. J. Romanow, N. Coustel, J. P. McCauley, N. C. Maliszewskyj, J. E. Fischer, and A. B. Smith, 1991, Phys. Rev. Lett. 67, 3808.

Nordman, C. E., and D. L. Schmitkons, 1965, Acta Crystallogr. 18, 764.

Normand, B. G. A., A. P. Giddy, M. T. Dove, and V. Heine, 1990, J. Phys. Condens. Matter 2, 3737.

Onsager, L., 1931a, Phys. Rev. 37, 1105.

Onsager, L., 1931b, Phys. Rev. 38, 2265.

Parsonage, N., and L. A. K. Staveley, 1978, Disorder in Crystals (Clarendon, Oxford).

Pick, R. M., and M. Yvinec, 1980, J. Phys. (France) 41, 1053.

Pintschovius, L., B. Renker, F. Gompf, R. Heid, S. L. Chaplot, M. Haluska, and H. Kuzmany, 1992, Phys. Rev. Lett. 69, 2662.

Press, W., 1973, Acta Crystallogr. A 29, 257.

Press, W., 1981, Single Particle Rotations in Molecular Crystals (Springer Tracts in Modern Physics No. 92) (Springer, New York).

Press, W., H. Grimm, and A. Hüller, 1979, Acta Crystallogr. A 35, 881.

Press, W., and A. Hüller, 1973a, Acta Crystallogr. A 29, 252.

Press, W., and A. Hüller, 1973b, Phys. Rev. Lett. 30, 1207.

Press, W., and A. Hüller, 1974, in Anharmonic Lattices, Structural Transitions and Melting, edited by T. Riste (Noordhoff, Leiden), p. 185.

Rand, S. C., and B. P. Stoicheff, 1982, Can. J. Phys. 60, 287.

Rehwald, W., 1973, Adv. Phys. 22, 721.

Rehwald, W., J. R. Sandercock, and M. Rossinelli, 1977, Phys. Status Solidi A 42, 832.

Richter, P. W., and C. W. F. T. Pistorius, 1972, J. Solid State Chem. 5, 276.

Rowe, J. M., J. Bouillot, J. J. Rush, and F. Luty, 1986, Physica B 136, 498.

Rowe, J. M., D. G. Hinks, D. L. Price, S. Susman, and J. J. Rush, 1973, J. Chem. Phys. 58, 2039.

Rowe, J. M., J. J. Rush, N. J. Chesser, K. H. Michel, and H. Naudts, 1978, Phys. Rev. Lett. 40, 455.

Rowe, J. M., J. J. Rush, D. G. Hinks, and S. Susman, 1979, Phys. Rev. Lett. 43, 1158.

Rowe, J. M., J. J. Rush, and E. Prince, 1977, J. Chem. Phys. 66, 5149.

Rowe, J. M., J. J. Rush, N. Vagelatos, D. L. Price, D. G. Hinks, and S. Susman, 1975, J. Chem. Phys. 62, 4551.

Sachidanandam, R., and A. B. Harris, 1991, Phys. Rev. Lett. 67, 1467.

Sachidanandam, R., and A. B. Harris, 1994, Phys. Rev. B 49, 2878. 
Sahu, D., 1986, J. Chem. Phys. 85, 5251.

Sahu, D., and S. D. Mahanti, 1982, Phys. Rev. B 26, 2981.

Sahu, D., and S. D. Mahanti, 1984, Phys. Rev. B 29, 340.

Sakurai, J., R. A. Cowley, and G. Dolling, 1970, J. Phys. Soc. Jpn. 28, 1426.

Salje, E., 1985, Phys. Chem. Miner. 12, 93.

Satija, S. K., and C. H. Wang, 1978, Solid State Commun. 28, 617.

Sawada, A., A. Hattori, and Y. Ishibashi, 1980, J. Phys. Soc. Jpn. 49, 423 (Lett.).

Sawada, S., S. Nomura, S. Fuji, and Y. Yoshida, 1958, Phys. Rev. Lett. 1, 320.

Schranz, W., A. Fuith, P. Dolinar, H. Warhanek, M. Haluska, and H. Kuzmany, 1993a, in Electronic Properties of Fullerenes, edited by H. Kuzmany, J. Fink, M. Mehring, and S. Roth (Springer-Verlag, Berlin), p. 177.

Schranz, W., A. Fuith, P. Dolinar, H. Warhanek, M. Haluska, and H. Kuzmany, 1993b, Phys. Rev. Lett. 71, 1561.

Seymour, R. S., and A. W. Pryor, 1970, Acta Crystallogr. B 26, 1487.

Shi, X. D., A. R. Kortan, J. M. Williams, A. M. Kini, B. M. Savall, and P. M. Chaikin, 1992, Phys. Rev. Lett. 68, 827.

Silvera, I. F., 1980, Rev. Mod. Phys. 52, 393.

Slonczewski, J. C., and H. Thomas, 1969, Phys. Rev. B 1, 3599.

Sprik, M., A. Cheng, and M. L. Klein, 1992a, J. Phys. Chem. 96, 2027.

Sprik, M., A. Cheng, and M. L. Klein, 1992b, Phys. Rev. Lett. 69, 1660 .

Stephens, P. W., L. Mihaly, P. L. Lee, R. L. Whetten, S. M. Huang, R. B. Kaner, F. Diederich, and K. Holczer, 1991, Nature 351, 632 .

Stephens, P. W., L. Mihaly, J. B. Wiley, S. M. Huang, R. B. Kaner, F. Diederich, R. L. Whetten, and K. Holczer, 1992, Phys. Rev. B 45, 5432.

Tanisaki, S., 1961, J. Phys. Soc. Jpn. 16, 579.

Terauchi, H., Y. Noda, and Y. Yamada, 1972, J. Phys. Soc. Jpn. 32, 1560.

Timmermans, J., 1961, J. Phys. Chem. Solids 18, 1.

Toledano, J-C., and P. Toledano, 1980, Phys. Rev. B 21, 1139.

Toupry, N., H. Poulet, M. Le Postollec, R. M. Pick, and M. Yvinec, 1983, J. Raman Spectrosc. 14, 166.

Tycko, R., R. C. Haddon, G. Dabbagh, S. H. Glarum, D. C. Douglass, and A. M. Mujsce, 1991, J. Phys. Chem. 95, 518.

Vagelatos, N., J. M. Rowe, and J. J. Rush, 1975, Phys. Rev. B 12,4522 . van Kranendonk, J., 1983, Solid Hydrogen (Plenum, New York/London).

Van Tendeloo, G., S. Amelinckx, J. L. De Boer, S. van Smaalen, M. A. Verheijen, H. Meekes, and G. Meijer, 1993, Europhys. Lett. 21, 329.

Vaughan, G. B. M., P. A. Heiney, D. E. Cox, J. E. Fischer, A. R. McGhie, A. L. Smith, R. M. Strongin, M. A. Cichy, and A. B. Smith III, 1993, Chem. Phys. 178, 599.

Von der Lage, F. C., and H. A. Bethe, 1947, Phys. Rev. 71, 612. Wagner, H., and J. Swift, 1970, Z. Phys. 239, 182.

Wakabayashi, N., B. Alefeld, W. Bührer, and H. G. Smith, 1982, Phys. Rev. B 26, 4160.

Weaver, J. H., D. M. Poirier, and Y. B. Zhao, 1993, in Electronic Properties of Fullerenes, edited by H. Kuzmany, J. Fink, M. Mehring, and S. Roth (Springer-Verlag, Berlin), p. 146.

Winter, J., and H. Kuzmany, 1992, Solid State Commun. 84, 935.

Wonneberger, S., and A. Hüller, 1987, Z. Phys. B 66, 191.

Yamada, Y., M. Mori, and Y. Noda, 1972, J. Phys. Soc. Jpn. 32, 1565.

Yamada, Y., Y. Noda, J. D. Axe, and G. Shirane, 1974a, Phys. Rev. B 9, 4429.

Yamada, Y., H. Takatera, and D. L. Huber, 1974b, J. Phys. Soc. Jpn. 36, 641.

Yannoni, C. S., R. D. Johnson, G. Meijer, D. S. Bethune, and J. R. Salem, 1991, J. Phys. Chem. 95, 9.

Yildirim, T., J. E. Fischer, A. B. Harris, P. W. Stephens, D. Liu, L. Brard, R. A. Strongin, and A. B. Smith, 1993, Phys. Rev. Lett. 71, 1383.

Yvinec, M., and R. M. Pick, 1980, J. Phys. (France) 41, 1045.

Zhou, O., J. E. Fischer, N. Coustel, S. Kyria, Q. Zhu, A. R. McGhie, J. P. Romanov, J. P. McCauley, A. B. Smith, and D. E. Cox, 1991, Nature 351, 462.

Zhou, O., Q. Zhu, G. B. M. Vaughan, J. E. Fischer, P. A. Heiney, N. Coustel, J. P. McCauley, A. B. Smith, and D. E. Cox, 1992, Mater. Res. Soc. Symp. Proc. 270, 191.

Zhu, Q., O. Zhou, Q. Zhu, J. E. Fischer, A. R. McGhie, W. J. Romanov, R. M. Strongin, M. A. Cichy, and A. B. Smith, 1993, Phys. Rev. B 47, 13948.

Zielinski, P., and K. Parlinski, 1984, J. Phys. C 17, 3301.

Zuk, J., D. M. Brake, H. Kiefte, and M. J. Clouter, 1989, J. Chem. Phys. 91, 5285.

Zuk, J., H. Kiefte, and M. J. Clouter, 1990, J. Chem. Phys. 92, 917. 